Mississippi State University

Scholars Junction

$8-6-2021$

\title{
Molecular dynamics study of structure-property relations of single-wall carbon nanotubes (SWCNT), partially unzipped carbon nanotubes (PUCNT), and damage evolution in nanocomposites of SWCNT with polyethylene (SWCNT-PE)
}

Sultana Abdullah Ababtin

sultana_1234@hotmail.com

Follow this and additional works at: https://scholarsjunction.msstate.edu/td

\section{Recommended Citation}

Ababtin, Sultana Abdullah, "Molecular dynamics study of structure-property relations of single-wall carbon nanotubes (SWCNT), partially unzipped carbon nanotubes (PUCNT), and damage evolution in nanocomposites of SWCNT with polyethylene (SWCNT-PE)" (2021). Theses and Dissertations. 5276. https://scholarsjunction.msstate.edu/td/5276

This Dissertation - Open Access is brought to you for free and open access by the Theses and Dissertations at Scholars Junction. It has been accepted for inclusion in Theses and Dissertations by an authorized administrator of Scholars Junction. For more information, please contact scholcomm@msstate.libanswers.com. 
Molecular dynamics study of structure-property relations of single-wall carbon nanotubes (SWCNT), partially unzipped carbon nanotubes (PUCNT), and damage evolution in nanocomposites of SWCNT with polyethylene (SWCNT-PE)

By

Sultana Abdullah Ababtin

Approved by:

Mark A. Novotny (Major Professor)

Mark F. Horstemeyer (Co-Major Professor)

Sara Adibi Sedeh

R. Torsten Clay

Steven R. Gwaltney

Youssef Hammi

Hendrik F. Arnoldus (Graduate Coordinator)

Jason M. Keith (Dean, Bagley College of Engineering)

\author{
A Dissertation \\ Submitted to the Faculty of \\ Mississippi State University \\ in Partial Fulfillment of the Requirements \\ for the Degree of Doctor of Philosophy \\ in Applied Physics \\ in the Department of Physics and Astronomy
}

Mississippi State, Mississippi

August 2021 
Copyright by

Sultana Abdullah Ababtin

2021 
Name: Sultana Abdullah Ababtin

Date of Degree: August 6, 2021

Institution: Mississippi State University

Major Field: Applied Physics

Major Professors: Mark F. Horstemeyer, Mark A. Novotny

Title of Study: Molecular dynamics study of structure-property relations of single-wall carbon nanotubes (SWCNT), partially unzipped carbon nanotubes (PUCNT), and damage evolution in nanocomposites of SWCNT with polyethylene (SWCNTPE)

Pages in Study: 91

Candidate for Degree of Doctor of Philosophy

This study employs the Modified Embedded Atom Method with Bond Order (MEAMBO) atomic potential as first used to capture single-wall carbon nanotube (SWCNT) properties accurately. We updated the MEAM-BO potential parameters to produce a MEAM-BO* potential for the SWCNT system. Calculating the wavenumber of the radial breathing mode (RBM), elastic properties, and folding energy $(\triangle \mathrm{E})$ of SWCNT which are all associated with bond curvature was considered. Interestingly, MEAM-BO* including the SWCNT data base, improved the previous fit of the $\mathrm{C}-\mathrm{H}$ system. Further discussed is how we constructed five different partially unzipped carbon nanotubes (PUCNT) and investigate the mechanical properties and self-healing of the simplest PUCNT by using molecular dynamics simulations. In addition, we studied the exploration of damage evolution and the mechanical response of a polyethylene/single wall carbon nanotube composite (SWCNT-PE) at different stress states, temperatures, and strain rates which are studied through utilizing atomistic simulations. The SWCNT-PE composite was expanded perpendicular to the nanotube direction and damage was computed in terms of the void number density (void nucleation) and void volume. 


\section{DEDICATION}

This research is first dedicated to my parents, Abdullah and Norah, whose words of encouragement have been pushing me along the way. They have been teaching me to believe in God then in myself, and to be strong and never give up easily.

To my husband, Falah Alshammari, who has been a mentor, friend, and supporter throughout all the difficulties I have gone through. He has made me a better person and made my dreams come true.

To our kids, Amal, Ryan, and Sultan. I know that you all have been missing me a lot. I am sorry for been busy with my work, but I am happy and proud of you all for depending on yourselves and doing your work, sometimes alone, and for taking responsibility and care of each other when your dad and I are busy or out for work.

To my siblings, who have helped and supported me in all kinds of ways. None of this work would be possible without my family's encouragement and support. 


\section{ACKNOWLEDGEMENTS}

I would like to first thank my advisors and guides, Dr. Mark Horstemeyer and Dr.Mark Novotny. Dr. Horstemeyer is definitely more than a great supervisor. He is an outstanding mentor and I'm excited to keep learning from him every day. He also really cares about his students and advises them all time to believe and trust god and themselves. After Dr. Horstemeyers had to leave MSU for Liberty University, he did his best to find someone as a great a supervisor for me and I was so lucky when Dr. Novotny accept to be my major advisor. I am very grateful for Dr. Novotny for working with me and providing me with support while I have been a student at MSU. For the continuous encouragement and guidance that you have given me in the last two years, I cannot express the depth of my gratitude. You were always there for me through the challenges of working toward my degree, and under your supervision, I believe that I have achieved the goals that we discussed from the beginning. I have learned more than expected and have enjoyed the process as a fulfilling and meaningful part of my journey.

I would also like to acknowledge all of my committee members for the guidance that you gave during this time of research. For all of the time and guidance that I received, I am eternally grateful. I appreciate the insights that you gave to me, and for the contributions that they made to my research. Thank you to Dr. Sara Adibi for being my supervisor at CAVS and supporting me there all the time. Dr. Steven Gwaltney, I am grateful for all of your explanations and insights into my research. Thank you to Dr. Youssef Hammi for your guidance and expertise into the ISV 
model. I would also like to thank Dr. R. Torsten Clay for his assistance with learning Computation physics and running simulations.

I would also like to thank Dr. Michael Baskes for the foundation that you provided for working with the MEAN potential that made this research a possibility.

To Dr. Haitham Elkadiri, I will never forget your support since when I was earning my master degree. You are the first person who opened a big gate in front of me for learning. you were the first reason that made me connect to the mechanical engineering department and join their big group, and introduced me to Dr. Horstemeyer and from here it was the first big step in my education life.

I am grateful for the insights and suggestions that came from Dr. Sungkwang Mun, and Dr. Andrew Bowman, which included being available for the questions that I had concerning the research and offering help when needed with the development of scripts, the process of running simulations, and the work of writing the paper.

As well, I am also grateful to my fellow graduate students who offered me friendship, a group with which to discuss research, further collaboration, and interactions that all had positive influences on the work. To Caleb Yenusah, I am appreciative for our discussion and encouragement to each other to reach the goals we wanted to achieve.

For Advanced Vehicular Systems (CAVS) and the research was sponsored by the Army Research Laboratory and was accomplished under Cooperative Agreement Number W911NF-152-0025. 


\section{TABLE OF CONTENTS}

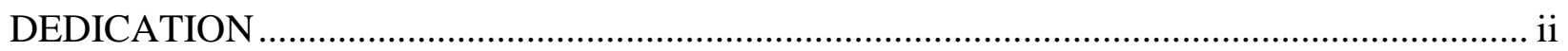

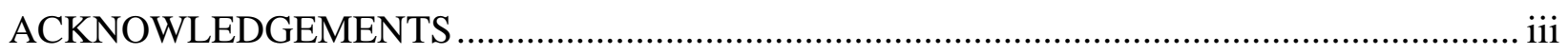

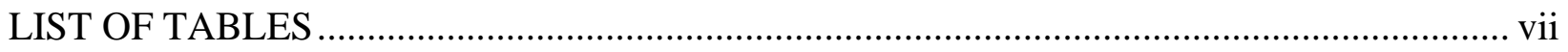

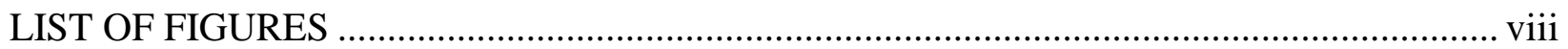

\section{CHAPTER}

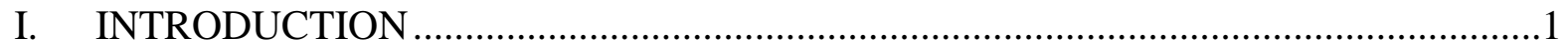

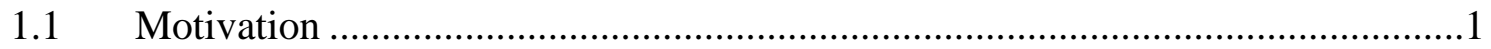

$1.2 \quad$ Structure of Single Wall Carbon Nanotube ………................................................

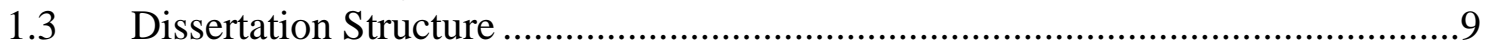

II. SINGLE-WALL CARBON NANOTUBE MECHANICAL BEHAVIOR USING THE MODIFIED EMBEDDED ATOM METHOD WITH BOND ORDER (MEAM-BO)..11

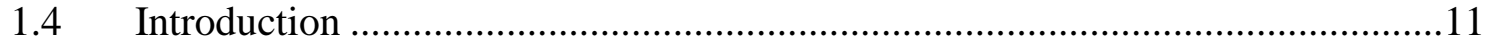

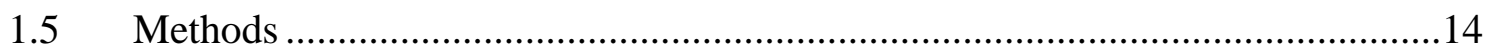

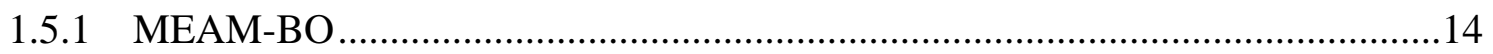

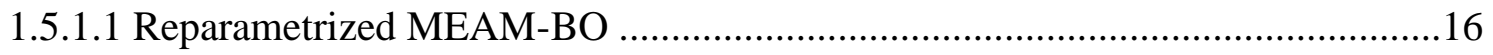

1.5.1.2 Parametrization procedure ........................................................................18

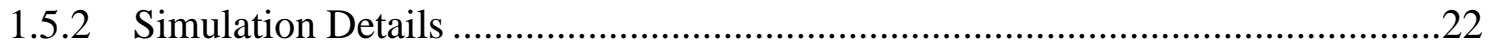

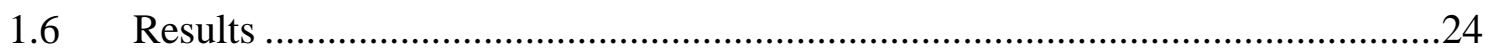

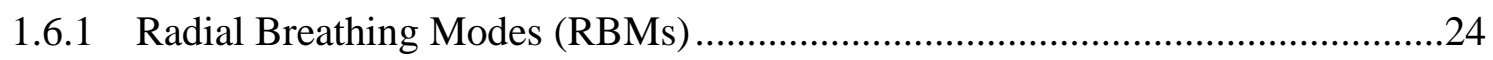

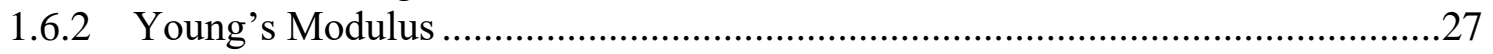

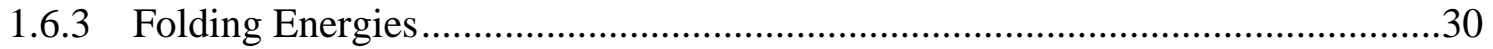

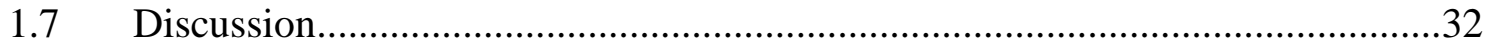

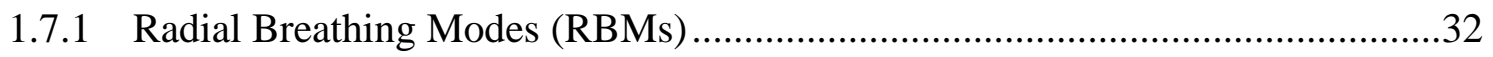

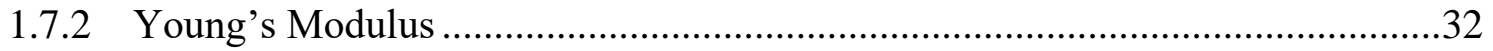

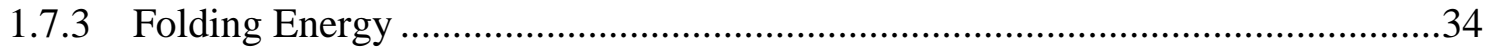

1.7.4 Accuracy of MD Simulation …………………….........................................34

1.7.5 Root Mean Square Error (RMSE) results of all the calculations. ........................35

III. MECHANICAL PROPERTIES OF DIFFERENT PARTIALLY UNZIPPED CARBON NANOTUBES BY ATOMISTIC SIMULATION ……………………....................... 


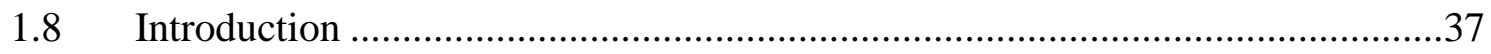

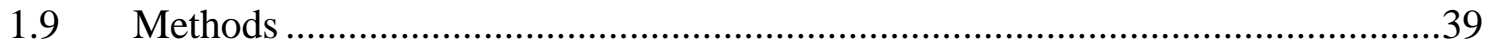

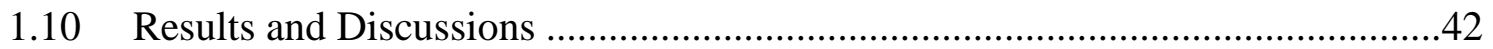

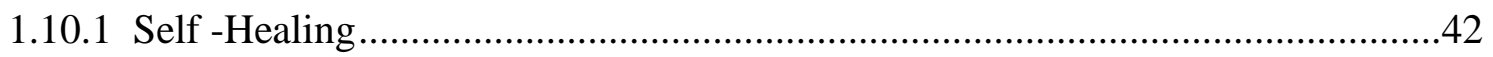

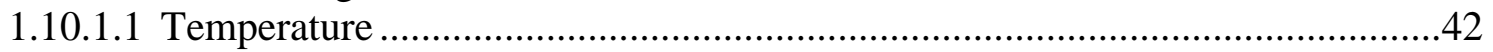

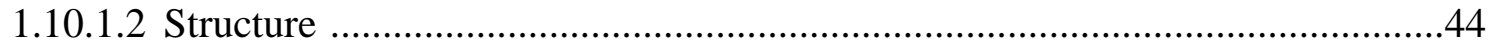

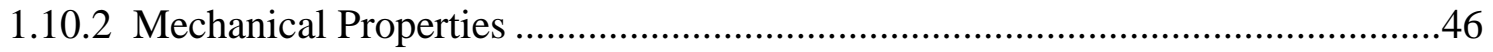

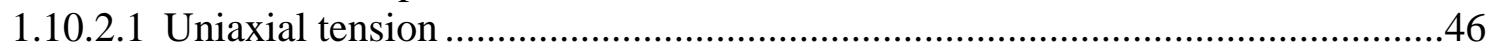

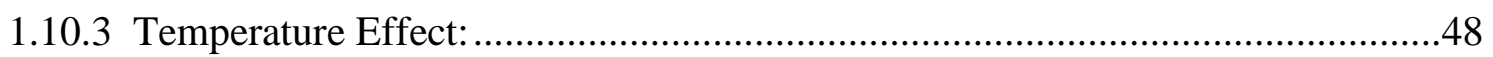

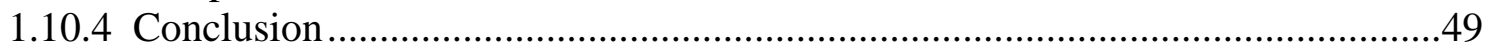

IV. DAMAGE EVALUATION IN POLYETHYLENE COMPOSITE FROM MOLECULAR DYNAMICS SIMULATION .......................................................51

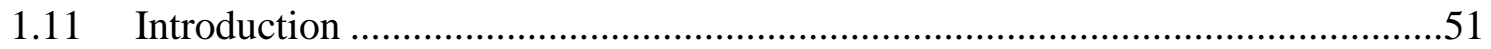

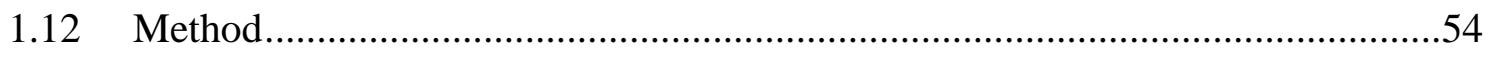

1.12.1 Model generation of SWCNT composite and Simulation Setup.......................54

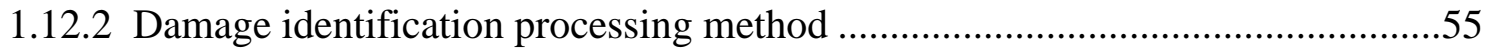

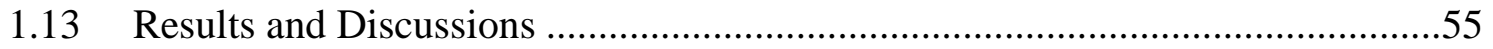

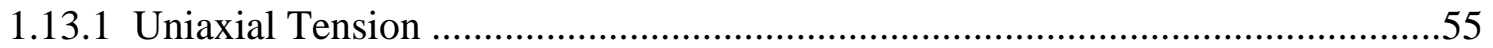

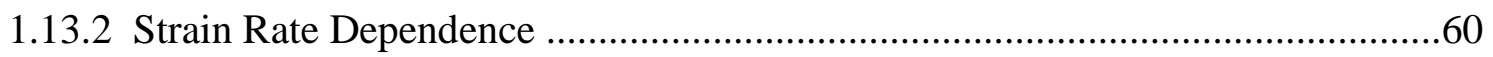

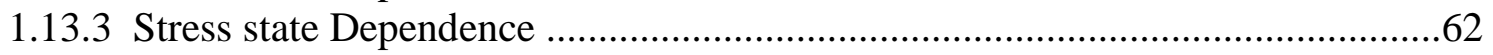

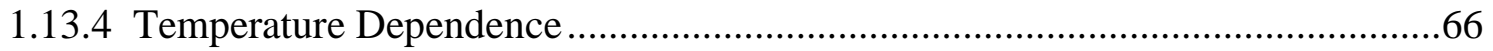

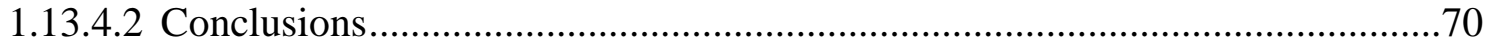

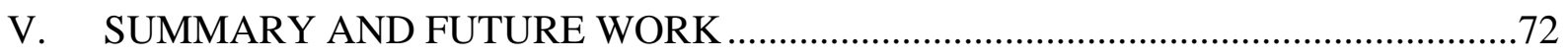

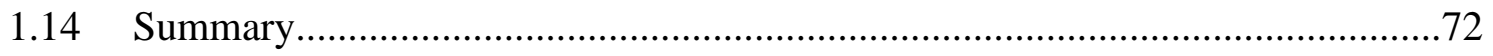

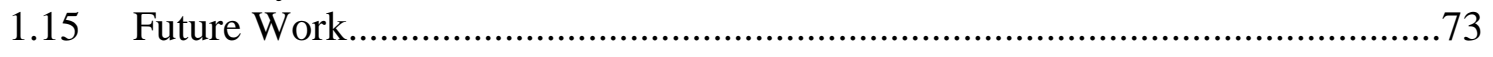

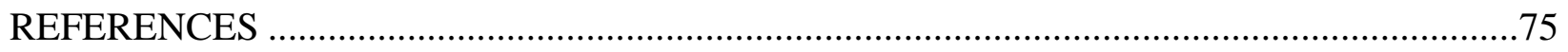

\section{APPENDIX}

A. GRAPHENE AND GRAPHITE PROPERTIES CALCULATED FROM MEAM-BO*

A.1 Graphene and graphite properties calculation 


\section{LIST OF TABLES}

Table 2.1 Parameters of MEAM-BO* and the van der Waals parameters..............................17

Table 2.2 Radial Breathing Mode results .......................................................................25

Table 2.3 The Second Derivative of the Energy Results...................................................29

Table 2.4 Root Mean Square Error (RMSE) of all the calculation for MEAM-BO, MEAM-BO*, REBO, and ReaxFF potentials..................................................35

Table 3.1 Overview of mechanical properties for different structures of unzipped CNT

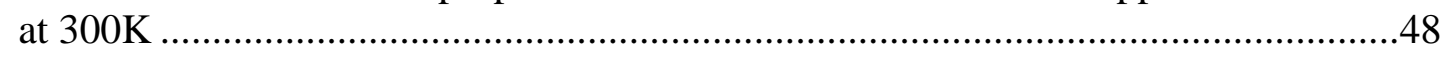

Table 3.2 Mechanical properties for 1st structure under different temperature ......................49

Table 4.1 Overview of mechanical properties for SWCNT-PE under different conditions

Table A.1 Graphene and graphite properties calculated from MEAM-BO (previous work $^{79}$ and current work) and REBO against the experimental data. The zero-point energy (ZPE) corrections used here are $0.166 \mathrm{eV} /$ atom for graphene and graphite ${ }^{143,144}$, respectively. ECorr is the corrected energy by ZPE 


\section{LIST OF FIGURES}

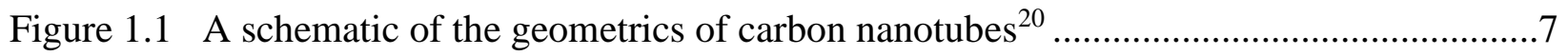

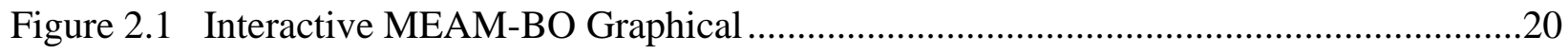

Figure 2.2 Screenshot for MEAM-BO user interface tools ................................................21

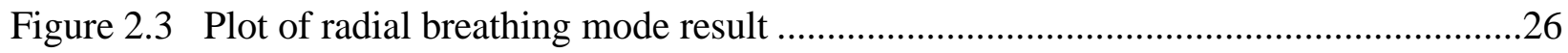

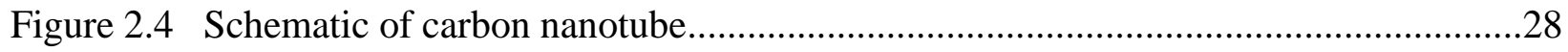

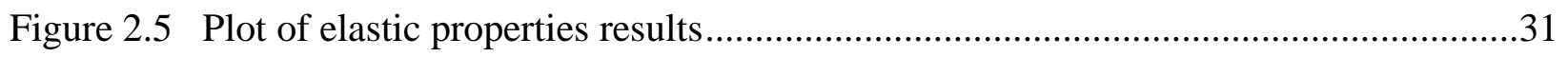

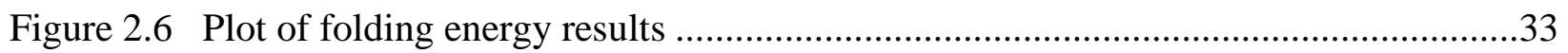

Figure 3.1 Schematic of five partially unzipped carbon nanotube (PUCNT)..........................41

Figure 3.2 Molecular dynamics (MD) simulation set-up for the unzipped CNT under uniaxial deformation in the $\mathrm{z}$ direction (vertical direction).

Figure 3.3 Partially unzipped $(12,12) \mathrm{CNT}$ vs. simulation time with cutting length $\mathrm{L}=$ $70(\AA)$, relaxed at different temperatures $400 \mathrm{~K}, 600 \mathrm{~K}$, and $1000 \mathrm{~K}$. a) unzipped length $b$ ) total energy

Figure 3.4 Structures of the first three partially unzipped $(10,10)$ CNT vs simulation time with cutting length relaxed at $1000 \mathrm{~K}$. a) unzipped length b) total energy

Figure 3.5 Stress-strain response for five partially unzipped carbon nanotube at $300 \mathrm{~K}$

Figure 3.6 Temperature effect on stress-strain curve of 1st structure

Figure 4.1 Stress-Strain curve response of stretched polyethylene composite with single wall carbon nanotube (SWCNT-PE) in y-direction

Figure 4.2 Damage evolution during uniaxial tensile deformation at $250 \mathrm{~K}$ illustrating (a) the stress-strain response, (b) void number density, (c) average void size, and $(\mathrm{d})$ the overall damage (void volume fraction). 
Figure 4.3 Stress-strain behavior of polyethylene composite beads SWCNT deformed at different strain rates at $250 \mathrm{~K}$

Figure 4.4 Damage evolution during uniaxial tensile deformation at various strain rate at a temperature of $250 \mathrm{~K}$ illustrating (a) void number density, (b) average void size, and (c) the overall damage (void volume fraction).

Figure 4.5 Equivalent stress-strain Curve at a temperature of $250 \mathrm{~K}$ and a strain rate of $3 \times 10^{9} / \mathrm{s}$. under different stress states

Figure 4.6 Damage evolution during different stress state with strain rate $3 \times 10^{9}$ showing (a) void number density, (b) average void size, and (c) the void volume fraction.

Figure 4.7 Dependent stress-strain response of SWCNT-PE at strain rate $3 \times 10^{9}$ .66

Figure 4.8 Damage evolution during uniaxial tensile with strain rate $3 \times 10^{9}$ at different temperatures showing (a) void number density, (b) average void size, and (c) the void volume fraction.

Figure A.1 The rotational barrier for ethylene calculated using MEAMBO(original/current) and FP using CCSD(2)/aug-cc-pVTZ. ${ }^{145,146}$ The white arrows in the pictures of the molecules indicate the coordinate that is being varied, and the line that passes through the FP or experimental data serves as a guide. The energy is relative to the minimum energy. 


\section{CHAPTER I}

\section{INTRODUCTION}

\subsection{Motivation}

In the $19^{\text {th }}$ century, Thomas A. Edison prepared the first carbon fiber to produce a filament for the electric light bulb ${ }^{1}$. Further research following this discovery focused on vapor grown carbon filaments, resulting in filament growth from the thermal decomposition of hydrocarbons. In the 1950s and 1960s, aerospace engineers sought strong, stiff, and light-weight fibers to make lightweight composite materials with superior mechanical properties. This goal led to an increased study of carbon fiber with polymers, thus improving its structure. Catalytic chemical vapor deposition (CVD) and vapor grown carbon (VGC) for synthesis of carbon fibers interested researchers, leading to growth in the commercialization of vapor grown carbon fibers for various applications in the 1990s. In 1985, Curl, Kroto and Smalley ${ }^{2}$ accidentally discovered a new structure, fullerenes, by vaporizing carbon in a helium atmosphere. This motivated studies about manufacturing a carbon filament of very small diameters. Dresselhaus proposed the building of carbon nanotubes (CNT) in August, 1991³; however, Iijima produced CNT experimentally for the first time by using transmission electron microscopy ${ }^{4}$.

CNTs can be used for many applications in both material science and engineering. NASA has used CNTs in developing materials for application in space exploration ${ }^{5}$. Creating bundles of Single Walled CNTs (SWCNTs) is considered the primary purpose for these tubes due to the high quality of transfer mechanisms, small bending stiffness, and the long-fatigue life ${ }^{6-8}$. Also, CNTs 
create multi-functionality in composite systems that are polymer based, which has inspired continuing research to improve the design of nano-composites. Further research suggests that the products can be used for voltage controlled nano metric actuators ${ }^{9}$, which might be needed in a nano-tweezer.

SWCNT can either be metallic or be a semiconductor, with the difference defined on the geometric structure based on the quantum confinement of electrons within the circumferential direction. Experimental devices have been able to use CNTs based on the basic nature of their properties for items such as delimiters, memory devices, and sensors. Commercial companies are working toward exploitation of the many possibilities for their use ${ }^{10}$.

Features on the nanoscale level of carbon nano tubes (CNTs) are of great importance and highly researched in the scientific community. The mechanical properties of CNTs support both applied research and theoretical exploration but are not completely understood. Practical investigations of CNTs require them to be interfaced with measurement equipment which is difficult. Manipulation and precise identification of CNTs can be quite challenging due to such limitations. In this chapter, we mainly focused on explaining observations, as well as obtaining information that is yet inaccessible through experimentation. The purpose of this dissertation is to examine opportunities for using innovative computational tools that are used to study CNTs as well as predicting and discovering their mechanical properties. In addition, analysis of related structures and learning more about nanotubes as research objects provides an opportunity to investigate new numerical approaches that are appropriate for finding solutions of problems that exist in the nanoscale world. The research also determines the comparison capability of different force fields and use of the modified embedded atom methods with bond order (MEAM-BO) potential as a base for further research projects on CNTs for the first time. Currently, researchers 
are interested in creating graphene nanosheets by using new cutting methods on CNTs to create graphene nanoribbons (GNRs). The potential for producing GNRs that can be used in an electronic device comes from the process of unzipping CNTs. As a result, a large amount of research has gone into combining CNTs and GNRs with the hope of exploiting their excellent mechanical, electronic, and magnetic properties. We are interested in using unzipped carbon nanotubes to enhance the strength of the interface structure of composites. We built different partially unzipped carbon nanostructures and investigated these structures by molecular dynamics and NonEquilibrium Green's Function methods (NEGF) to understand their behavior.

The study of nanocomposites of carbon nanotubes has spanned theoretical and experimental research to understand and characterize behavior based on mechanical effects. It is also helpful to study the specific development of fracture mechanisms and fracture formation, which is one example in the study of interfacial properties. Present research has not yet provided a full understanding of how these effects occur. The best understanding of these effects is found in the evidence of nanocomposite behavior in complex mechanical systems. This complex mechanical system depends on constituent materials and the way those materials are distributed.

No current research is available for large-scale Molecular Dynamic (MD) simulations of interfacial deformation of nanocomposite carbon nanotubes with polymers with reactive, bondbreaking, and unsaturated hydrocarbons potentials. The formulation of a computationally efficient MEAM-BO potential opens new doors for the simulation of nanocomposite polymeric systems by allowing for a superior description of free surfaces with inherent bond break capabilities. It also allows for an accurate description of the free volume evolution that plays a vital role in capturing the deformation and fracture behavior of interfacial and entire nanocomposites. 


\subsection{Structure of Single Wall Carbon Nanotube}

Carbon nanotubes (CNTs) are cylindrical objects with a diameter of a few nanometers that are formed when sheets of hexagonally arranged carbon atoms are rolled. These tubes may be linked along their lengths to form elongated tubes measuring several microns in length or run along the same axis in the form of coaxial tubes. The fundamental form of these CNTs is the single-walled CNT (SWCNT), but complex forms made of many walls, called multi-walled CNTs (MWCNTs), have also been described. In the one-dimensional form, all atoms of the CNT crystal are surface atoms, and its mechanical and electrical properties are similar to those of a graphene sheet. This resemblance makes it possible to use the structure of two-dimensional graphene sheets to model the structure of the SWCNTs.

Graphene, the two dimensional form of graphite, has been widely studied in theory and experimentally ${ }^{11-17}$, making it a good model for studying the structures of compounds believed to have a similar structure, such as CNTs and fullerenes. High resolution electron microscopy approaches show that atoms in graphene are arranged in a honeycomb structure with stacked layers that have a spacing of $3.35 \AA$, and carbon atoms that are $1.42 \AA$ away from their nearest neighbor $\left(a_{0}\right)^{18}$. The small distance between neighboring carbon atoms is the smallest distance of solid elements at room temperature and the large spacing between the layers makes it occur without significant mechanical, chemical, or electrical changes on its surface atoms. These properties enable the study of the properties of graphite and CNTs based on the study of graphene $^{19}$.

A mathematical description of the graphene sheet and thus of a CNT can be developed based on a two-dimensional hexagonal unit cell using vectors. The point $\mathrm{O}$ denotes the origin in 
the sheet. Vectors $\mathbf{a}_{1}$ and $\mathbf{a} 2$ are the primitive vectors which can be used to define the positions $\mathbf{P}_{\mathbf{1}}$ and $\mathbf{P}_{2}$ of two atoms joined by a covalent bond on a graphene sheet in its ground level.

$$
\begin{aligned}
& P_{1}=\frac{\left(a_{1}+a_{2}\right)}{3} \\
& P_{2}=\frac{2\left(a_{1}+a_{2}\right)}{3}
\end{aligned}
$$

The two vectors shown in (1.1) could be specified to enable the determination of the chiral structure of SWCNTs in Eq (1.2)

$$
\begin{array}{r}
\boldsymbol{a}_{1}=a_{0}\left(\frac{\sqrt{3}}{2} \widehat{e_{1}}+\frac{3}{2} \widehat{e_{2}}\right) \\
\boldsymbol{a}_{2}=a_{0}\left(\frac{-3}{2} \widehat{e_{1}}+\frac{\sqrt{3}}{2} \widehat{e_{2}}\right)
\end{array}
$$

The distance between two carbon atoms $\left(a_{0}\right)$ is estimated as $1.42 \AA$ at room temperature ${ }^{18}$, and $\widehat{e_{1}}$ and $\widehat{e_{2}}$ are unit vectors along the $x_{1}$ and $x_{2}$ axes as shown in figure (1.1).

The area of the graphene unit cell $S_{G}$ for the basis vectors is determined by

$$
S_{G}=\left|\boldsymbol{a}_{\mathbf{1}} \times \boldsymbol{a}_{\mathbf{2}}\right|=\frac{3 \sqrt{3} a_{0}^{2}}{2}
$$

The chiral structure of SWCNTs is defined based on the compounds' helicity and radius, which necessitates the use of a pair of integers $(n, m)$ that lead to a chiral vector $\left(\boldsymbol{C}_{\boldsymbol{h}}\right)$ defined by

$$
\boldsymbol{C}_{\boldsymbol{h}}=n \boldsymbol{a}_{1}+m \boldsymbol{a}_{2}
$$

which shows the chiral vector to be a function of the defined integers and the vectors $\mathbf{a}_{1}$ and $\mathbf{a}_{2}$ 
specified above. The chiral vector corresponds to the diameter of the CNT, and connects two points $\left(\mathbf{P}_{1}\right.$ and $\left.\mathbf{P}_{3}\right)$ that are crystallographically equivalent on the graphene sheet's lattice. As such, it could also be expressed as a vector acting on $\mathbf{P}_{\mathbf{1}}$ to get to $\mathbf{P}_{\mathbf{3}}$, as indicated in the equation

$$
C_{h}=\overline{P_{1} P_{3}}
$$

Each pair of the integers used shows how the sheet could be rolled to give a SWCNT. To prevent specifications that would lead to overlapping of the regions for rolling the graphene sheet, a constraint is imposed such that the second integer $(m)$ must always be equal to or lesser than the first integer $(n)$. Imposing this constrain ensures that every SWCNT is specified only once. This integer pair defines the chiral angle $(\theta)$ and diameter $(d)$ of the CNT with the chiral angle being the arctangent of the relationship between the two integers as shown

$$
\begin{gathered}
\tan \theta=\frac{\sqrt{3} m}{2 n+m} \\
d=\frac{\sqrt{3} a_{0}}{\pi}\left(n^{2}+m^{2}+n m\right)^{\frac{1}{2}}
\end{gathered}
$$

The diameter is a function of the distance between two adjacent carbons $\left(a_{0}\right)$ and the integers expressed in the form illustrated in in Eq (1.6). 


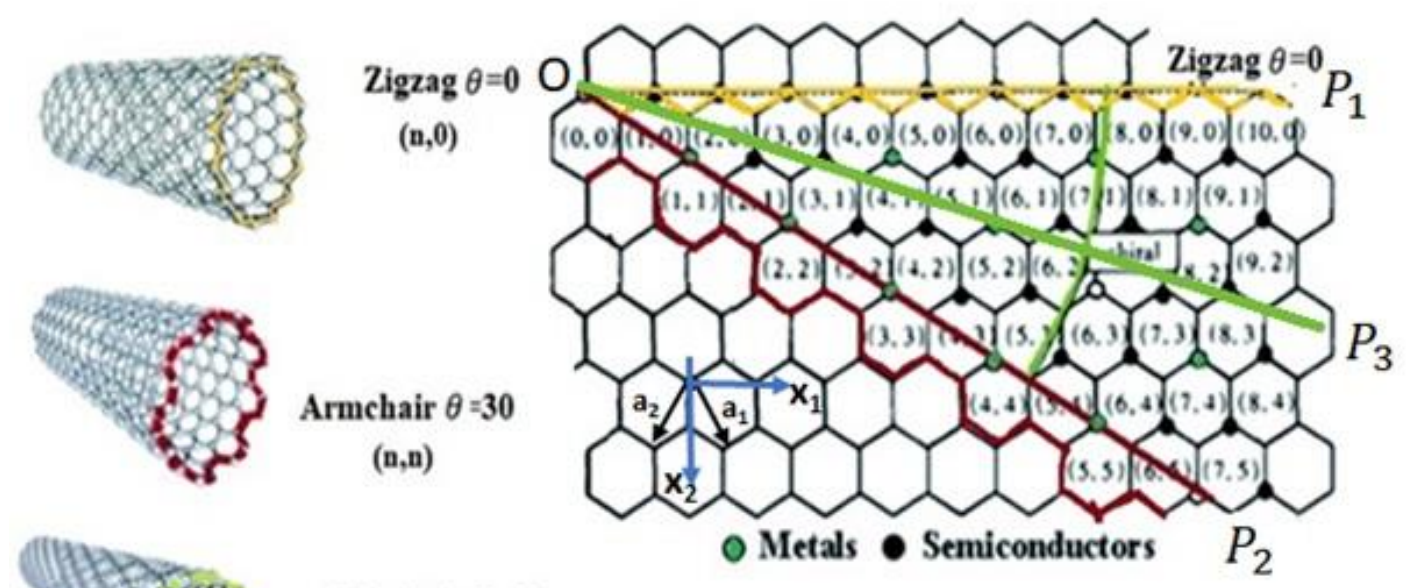

Chiral $0>\theta>30$

$(\mathrm{n}, \mathrm{m})$

Figure 1.1 A schematic of the geometrics of carbon nanotubes ${ }^{20}$

However, as seen in Eq (1.7) this distance is influenced by the radius of the tubule $\left(\frac{\sqrt{3}}{\pi}\right)$, leading to an estimated distance between the adjacent carbons of $1.42 \AA$ in a SWCNT ${ }^{18}$. Since the length of the chiral vector represents the diameter of the tube, Eq (1.7) can be simplified in terms of this vector. The approximations provided by simplifying (1.7) are not usually accurate values for the tubule diameter because they do not account for the curvature of the cylindrical surface and how it affects the conformation in the ground state. Based on the diameter and the chiral angle, three types of SWCNTs can arise, with their classification being defined by their chirality. The 'zigzag' nanotubes arise from a chiral angle equal to zero, whereas the 'armchair' nanotubes arise when the chiral angle is equal to the diameter of the tubule. The third type, the 'generic chiral' nanotubes, are described by a chiral angle greater than one but smaller than the tubule diameter. 
The formulation of the chiral angle is based on the roll-up angle, which forms between the chirality vector and the first vector specified in (1.2), which then leads to this expression

$$
\cos \theta=\frac{a_{1} \cdot C_{h}}{\left|a_{1}\right|\left|C_{h}\right|}
$$

Based on this relationship, a CNT forms as a one-dimensional compound with a roll-up angle of $0 \leq \theta \leq \frac{\pi}{6}$, implying that forming a CNT from a unit cell requires only one vector, the translational vector $\left(\boldsymbol{T}_{\boldsymbol{h}}\right)$, which transects the chirality vector along a $90^{\circ}$ angle. The translational vector is specified by

$$
\boldsymbol{T}_{\boldsymbol{h}}=t_{1} \boldsymbol{a}_{1}+t_{2} \boldsymbol{a}_{2}
$$

The two coefficients are determined using the hcd, which stands for the highest common divisor, as

$$
t_{1}=\frac{(n+2 m)}{h c d(n+2 m, 2 n+m)}, \text { and } t_{2}=\frac{-(2 n+m)}{h c d(n+2 m, 2 n+m)}
$$

The area of a graphene cell $S_{G}$ can be expressed in terms of the vectors described above, which also enables the expression of the area of the SWCNT unit cell $\left(S_{T}\right)$ is given by

$$
S_{T}=\left|\boldsymbol{T}_{\boldsymbol{h}}\right|\left|\boldsymbol{C}_{\boldsymbol{h}}\right|=\frac{\sqrt{3} a_{0}\left(n^{2}+m^{2}+n m\right)}{h c d(n+2 m, 2 n+m)}
$$

Therefore, the number of atoms $N_{T}$ per unit cell of the SWCNT can be obtained by dividing the area of the SWCNT unit cell $S_{T}$ by the area of the graphene cell $S_{G}$. This number helps determine the electronic properties of SWCNTs based on the known properties of graphene

$$
N_{T}=2 \frac{S_{T}}{S_{G}}=\frac{4\left(n^{2}+m^{2}+n m\right)}{h c d(n+2 m, 2 n+m)} .
$$




\subsection{Dissertation Structure}

The motivation for this research on structure of CNTs can be found in the overview that is presented in Chapter I. Chapter II presents the methods performed for different force fieldbased MD simulations to calculate the wavenumber of the Radial Breathing Mode (RBM), Young's moduli, and folding energies for various armchair single-wall nanotubes (SWCNTs). The reliability of the MEAMBO potential was checked by using the elastic coefficient calculation of the nanotubes without any consideration of nanotube thickness. The elastic (Young's) modulus was calculated by using the second derivative of energy with respect to the

axial strain $\left(\frac{\partial E^{2}}{\partial \varepsilon^{2}}\right)$. The Modified Embedded Atom Method with Bond Order (MEAM-BO), REBO, and ReaxFF are the reactive force fields we used to validate the experimental data and theoretical calculations to see how the potentials have the capability and accuracy to capture SWCNT characteristics.

As this is the first work using the MEAM-BO potential to model SWCNTs, it is important to set and modify the MEAM-BO potential parameters MEAM-BO* for a SWCNT and use it for unzipped SWCNT and SWCNT composite with polymers in Chapters III and IV.

Chapter III presents five different partially unzipped carbon nanotubes (PUCNT). The study was constructed to investigate the mechanical properties and temperature effect on selfhealing of the simplest PUCNT by using molecular dynamics simulations.

The parametric study that is found in Chapter IV provides an exhaustive review of how strain rate, stress states, as well as temperature has an effect on plasticity, fracture response, and damage of the entire the SWCNT composite. Stress and strain behaviors studied regarding each of the parameters that have been selected for comparison, as well as evaluations made regarding the evolution of void nucleation, coalescence, and growth, which were also compared. 
The final chapter provides a summary of the research results, with recommendations for how future work can explore additional characteristics and various aspects of the findings. 


\section{CHAPTER II}

\section{SINGLE-WALL CARBON NANOTUBE MECHANICAL BEHAVIOR USING THE MODIFIED EMBEDDED ATOM METHOD WITH BOND ORDER (MEAM-BO)}

\subsection{Introduction}

Since Iijima ${ }^{4}$ initially discovered carbon nanotubes (CNTs), nanotubes have received much attention in both experimental ${ }^{7,21-31}$ and theoretical studies ${ }^{32-42}$ because of their unique mechanical and electronic properties. Carbon nanotubes have a distinctive geometric structure with unsaturated bonds in small diameters that present challenges for developing force fields for Molecular Dynamics (MD) simulations that accurately capture their properties ${ }^{35-43}$. One method to examine the force field capability of capturing CNT properties is to compare the results of Radial Breathing Mode (RBM) simulations with the results from Raman spectroscopy, which is a common method used to provide bonding and characterization of CNTs and associated RBM frequencies. Homogeneously deforming a CNT in the radial direction gives a particular frequency of the RBM, which is considered a fingerprint of each CNT. Previous experimental studies ${ }^{30,31,43-46}$ have investigated radial vibrations using Resonant Raman Scattering. Radial vibrations have also been theoretically investigated by using Density Functional Theory (DFT) $37,38,40,47$ and semi-empirical Tight-Binding Molecular Dynamics (TBMD) ${ }^{48-51}$. In addition, MD simulations were used to determine the RBM frequencies of a diverse range of $\mathrm{CNTs}^{52-}$ 
55. The RBM frequency $\left(\omega_{R B M}\right)$, presented in terms of wavenumber $\boldsymbol{v}\left(\mathrm{cm}^{\mathbf{- 1}}\right)$, was inversely proportional to the tube diameter $(d)$. The radial breathing mode and the nanotube diameter have an inverse relationship ${ }^{56}$ given by the following

$$
v_{R B M}=\frac{A}{d}
$$

where $A$ is the proportionality constant ${ }^{57}$. This relationship is not likely to hold for tube diameters less than $7.6 \AA$ as a result of the curvature effects ${ }^{38,40,58-62}$. Lawler et $a l .{ }^{40}$ modified Eq.(2.12), by assuming that dependence of the strain energy with the inverse square of the nanotube's diameter extends to a continuous strain amplitude as shown below,

$$
v_{R B M}=\frac{A}{d}+\frac{B}{d^{3}}
$$

Eq.(2.2) enhances the radial breathing mode wavenumber results for small diameters. Lawler and his group ${ }^{40}$ performed DFT calculations and found values $A=2260 \AA \mathrm{cm}^{-1}$ and $B=$ $15 \pm 0.5 \AA^{3} \mathrm{~cm}^{-1}$ using Eq.(3.2) and $A=2310 \AA \mathrm{cm}^{-1}$ using Eq.(2.12). In other studies, Kürti et al. $^{37}$ applied the local density approximation to Eq.(2.1) and Longhurst et al. ${ }^{52}$ applied the REBO potential calculation to Eq.(2.1) to show that RBM frequencies are independent of the chiral angle $\theta$ and the nanotube length and found that $A$ equaled $2360 \AA \mathrm{cm}^{-1}$ and $2120 \AA \mathrm{cm}^{-1}$, respectively (a 10\% difference). Bandow et al. ${ }^{63}$ determined that $A$ equaled $2237.5 \AA \mathrm{cm}^{-1}$ by applying the constant force model interacting with the fourth neighbor. Alvarez et al. ${ }^{64}$ highlighted that $A$ equaled $2320 \AA \mathrm{cm}^{-1}$ for a crystal packing of identical infinite nanotubes using the Lennard-Jones potential ${ }^{65}$ to calculate the tubal van der Waals interaction. Other values for $A$ include $2225 \AA \mathrm{cm}^{-1}$ using the zone folding method ${ }^{31}$ and $2180 \AA \mathrm{cm}^{-1}$ using the force constant model. ${ }^{6}$ 
In addition to the great interest in studying calculations for the RBM frequency of a single-wall carbon nanotube (SWCNT), studying the elastic properties of a SWCNT is also of great interest in order to make and use them. Nanotubes are considered an incredible composite material choice due to their low weight and very high Young's modulus; however, experimental and theoretical measurements show considerable uncertainty in their results for SWCNTs. There are different reasons for such uncertainty, such as the small dimensions of nanotubes ${ }^{67}$, insufficient numbers of atoms being used to describe their small size ${ }^{68}$, and variation in the definition of thickness values and Young's modulus ${ }^{34,36,69-72}$. Treacy et al. ${ }^{67}$ calculated the Young's modulus for a different nanotube by using thermal vibrations and obtained the average value of Young's modulus as $1.8 \mathrm{TPa}$. In addition, Overney et al. ${ }^{68}$ reported a Young's modulus value of $5 \mathrm{TPa}$ for a capped single-wall carbon nanotube $(5,5)$ using an empirical Keating force constant model ${ }^{73}$. Similarly, based on the Tersoff-Brenner's potential ${ }^{74,75}$, Yakobson et al. $^{72}$ determined Young's modulus to be $5.5 \mathrm{TPa}$, well aligned with continuum elasticity theory.

Another important attribute of a SWCNT is the folding energy $(\triangle E)$ and the influence of the diameter thereupon. The folding energy is defined as the energy difference between the tube and a graphene sheet ${ }^{37,38,76}$ or graphite ${ }^{77,78}$ per carbon atom. Kürti et al. ${ }^{37}$ generalized the behavior of the folding energy with the carbon nanotube diameter where the folding energy increased for all nanotubes with a diameter greater than $10 \AA$ as an inverse square of the diameter according to continuum elastic theory. The proportionality constant $C_{1}$ obtained was 7.8 $\mathrm{eV} \AA^{2}$. Conversely, for diameters less than $10 \AA$, they reported that the folding energy increased faster than the inverse square. Kanamitsu et al. ${ }^{76}$ and Sawada et al. ${ }^{77}$ showed how the folding energy increased faster with diameters smaller than $10 \AA$ by fitting their data to 


$$
\triangle E=\frac{C_{1}}{d^{2}}+\frac{C_{2}}{d^{4}}
$$

where $C_{1}=6.85 \mathrm{eV}^{2}$ and $C_{2}=20.02 \mathrm{eV} \AA^{4}$ for the Kanamitsu et al. ' $s^{76}$ results, and $C_{1}=5.64$ $\mathrm{eV} \AA^{2}$, and $C_{2}=25.1 \mathrm{eV} \AA^{4}$ for the Sawada et al. 's $s^{77}$ results.

In this work, we performed different force field-based MD simulations to calculate the wavenumber of RBM, Young's moduli, and folding energies for various armchair single-wall nanotubes (SWCNTs). The reliability of the MEAM-BO potential was checked by using the elastic coefficient calculation of the nanotubes without any consideration of the nanotube thickness. The elastic (Young's) modulus (Y) was calculated by using the second derivative of the energy with respect to the axial strain $\left(\partial^{2} E / \partial^{2} \varepsilon\right)$. The Modified Embedded Atom Method with Bond Order (MEAM-BO $)^{79}, \mathrm{REBO}^{80}$, and $\mathrm{ReaxFF}^{81}$ are the reactive force fields we used to validate the experimental data and theoretical calculations to see how the potentials have the capability and accuracy to capture SWCNT characteristics. Then we updated MEAM-BO's parameters to improve the accuracy of the original MEAM-BO and produce a modified version (MEAM-BO*) for CNT systems.

\subsection{Methods}

\subsubsection{MEAM-BO}

Over the years, Baskes et al. ${ }^{75,82}$ developed and applied the Modified Embedded Atom Method (MEAM) to different mechanical behaviors: interface debonding of a biomaterial ${ }^{83}$, fracture of metals ${ }^{84}$, fracture of hydrocarbons ${ }^{85}$, fatigue crack growth ${ }^{86,87}$, and fragmentation ${ }^{88}$. Since the context of using the SWCNTs is to use them as strengtheners in composite materials, understanding the swelling, bending, and frequency response are key parameters to fracture behavior. Previously, Mun from the Baskes' group. ${ }^{79}$ reported MEAM with bond order (MEAM- 
BO) that was able to describe both saturated and unsaturated hydrocarbons. This is an improvement from $\mathrm{MEAM}^{89}$, which was only able to describe saturated hydrocarbons. It should be noted that MEAM-BO is compatible with the original MEAM because without changing the original MEAM equations, the energies regarding the bond order have been added. It means that MEAM-BO can be used with the other parameters from the literature, especially of various metallic systems. Below is a summary of the potential formalisms of MEAM and its bond order terms. In MEAM, the system's energy for an atom of type $\tau$ is given by the following equation,

$$
E_{M E A M}=\sum_{\mathrm{i}} \mathrm{F}_{\tau_{i}}\left(\bar{\rho}_{\mathrm{i}}\right)+\frac{1}{2} \sum_{\mathrm{j} \neq \mathrm{i}} \mathrm{S}_{\mathrm{ij}} \varphi_{\mathrm{ij}}\left(\mathrm{R}_{\mathrm{ij}}\right)
$$

where the embedding function $\mathrm{F}_{\tau_{i}}$ is the energy cost to atom $i$ in a location with the background electron density $\left(\bar{\rho}_{i}\right), S_{i j}$ is the screening function that identifies the three-body interactions, and $\varphi_{i j}$ is the pair interaction term, which one obtains by finding the universal equation of state $(\mathrm{UEOS})^{90}$ of a specified reference structure with nearest neighbor distance $R_{i j}$.

Some modifications were added to $\mathrm{MEAM}^{59}$ to include use of a bond order term. MEAM-BO includes energy fractions for double bonds $f_{I 2}$ with a double bond energy $E_{2}\left(R_{I}, B O_{I}\right)$ and energy fractions for a triple bond $f_{I 3}$ with triple bond energy $E_{3}\left(R_{I}\right)$. For an ideal double bond, $f_{I 2}$ is 1 and $f_{I 3}$ is equal to 0 , and the inverse is valid for a triple bond. The formalism of MEAM-BO is given by the following equation,

$$
E_{M E A M-B O}=E_{\mathrm{MEAM}}+\sum_{I} f_{I 3} \cdot E_{3}\left(R_{I}\right)+\sum_{I} f_{I 2} \cdot E_{2}\left(R_{I}, B O_{I}\right)
$$

where the double bond's energy fraction term in the above equation is given by

$$
\begin{gathered}
f_{12}=S_{i_{1} i_{2}} \cdot D_{2}^{(0)}\left(\left[Z_{i_{1}}^{(0)}-Z_{2}^{(0)}\right]^{2}\right) \cdot D_{2}^{(0)}\left(\left[Z_{i_{2}}^{(0)}-Z_{2}^{(0)}\right]^{2}\right) \\
. D_{2}^{(1)}\left(\left[Z_{i_{1}}^{(1)}\right]^{2}\right) \cdot D_{2}^{(1)}\left(\left[Z_{i_{2}}^{(1)}\right]^{2}\right) \cdot D_{2}^{(3)}\left(\left[Z_{I}^{(3)}\right]^{2}\right) \\
+f_{13} \cdot\left(1-D_{3}^{(3)}\left(\left[Z_{I}^{(3)}\right]^{2}\right)\right)
\end{gathered}
$$


$S_{i_{1} i_{2}}$ serves as the angular screening atomic function within the bond. The partial density functions are $Z_{i_{1}}^{(0)}, Z_{i_{1}}^{(1)}$, and $Z_{I}$ which enable an atom $i$ or a bond $I$ to satisfy the geometric requirements of a reference structure. $D_{b o n d}^{(h)}$ is a Gaussian function where the center of the peak is at the value zero. The equation's final part highlights the transformation process from a triple to a double bond (see Mun et al. ${ }^{79}$ and Dickel et al. ${ }^{91}$ for further details).

\subsubsection{Reparametrized MEAM-BO}

As part of the MEAM-BO formalism, a van der Waals (vdW) type interaction is explicitly included for the carbon-carbon (C-C) interactions between two adjacent layers of a graphite structure. It should be noted that the vdW term used in this work is only for C-C interactions, while a more general form is used in sulfur systems by adapting a DFT-D3 correction as in the work of Dickel et al. ${ }^{91}$. Here, we used the C-C vdW term that is initially developed for hydrocarbon systems. Also, because CNTs were not part of the fitting database and newly considered in this work, reparameterization was done, especially for bond order parameters. Thanks to the additive characteristic of bond-order energies, the MEAM parameters for saturated bonded interaction remained unchanged. Change has been made to four double bond parameters and two C-C vdW parameters to correct some of the SWCNT properties, such as the folding energy and RBM frequencies. The relevant equation for the double bond parameters is where $\beta_{\text {bond }}^{(h)}$ and $P_{\text {bond }}^{(h)}$

$$
\mathrm{D}_{2}^{(\mathrm{h})}(\mathrm{z})=\exp \left(-\mathrm{Z}^{\mathrm{P}_{2}^{(\mathrm{h})}} \cdot \beta_{2}^{(\mathrm{h})}\right)
$$

represent six adjustable parameters $(\mathrm{h}=1,2,3)$ for the double bond (bond $=2$ ). 
Table 1.1 Parameters of MEAM-BO* and the van der Waals parameters

\begin{tabular}{|c|c|c|c|c|c|c|c|c|c|c|c|c|c|c|}
\hline \multirow[b]{2}{*}{ Bond Type } & \multirow[b]{2}{*}{$B O$} & \multirow[b]{2}{*}{$R_{\text {bond }}$} & \multirow[b]{2}{*}{$\beta_{\text {bond }}^{(0)}$} & \multirow[b]{2}{*}{$\beta_{\text {bond }}^{(1)}$} & \multirow[b]{2}{*}{$\beta_{\text {bond }}^{(2)}$} & \multirow[b]{2}{*}{$\beta_{\text {bond }}^{(3)}$} & \multirow[b]{2}{*}{$p_{\text {bond }}^{(0)}$} & \multirow[b]{2}{*}{$p_{\text {bond }}^{(1)}$} & \multirow[b]{2}{*}{$p_{\text {bond }}^{(3)}$} & \multirow[b]{2}{*}{$k / l$} & \multirow[b]{2}{*}{$r_{l}$} & \multicolumn{3}{|c|}{$g_{k l} / e_{k}$} \\
\hline & & & & & & & & & & & & 0 & 1 & 2 \\
\hline & 2 & 1.339 & & & & & & & & 0 & 1.3396 & -1.295 & -4.53 & -10.754 \\
\hline \multirow[t]{2}{*}{ double } & $3 / 2$ & 1.397 & 5 & 0.4 & 4.50 & 0.6 & 1 & 1 & 1.7 & 1 & -0.0931 & -0.651 & -3.656 & -14.492 \\
\hline & $4 / 3$ & 1.420 & & & & & & & & 2 & 0.0455 & 0.285 & -4.287 & -19.884 \\
\hline triple & 3 & 1.203 & 5 & 5 & 4.50 & 0.46 & 1 & 1 & 1 & - & - & -3.648 & -2.981 & -13.146 \\
\hline
\end{tabular}

a. MEAM-BO bond parameters for the double bond with ethylene, benzene, and graphene reference structures and for the triple bond with acetylene reference structure. The bond order $B O$ and the experimental C-C bond lengths $\boldsymbol{R}_{\text {bond }}$ are given. The $\boldsymbol{r}_{\boldsymbol{l}}$ are expansion parameters for the determination of the double bond length, and the $\boldsymbol{g}_{\boldsymbol{k} \boldsymbol{l}}$ are expansion parameters for the double bond energy increments. The $\boldsymbol{e}_{\boldsymbol{k}}$ are expansion parameters for the triple bond increments. The $\boldsymbol{\beta}_{\boldsymbol{b o n d}}^{(\mathbf{0 , 1}, \mathbf{3})}$ are the exponential decay factors for the $D(z)$ function and bond increment function. The $\boldsymbol{p}_{\text {bond }}^{(\mathbf{0 , 1}, \mathbf{3})}$ are the power in the $D(z)$ function. See Mun et al [80] for the equations. Changes to the original MEAM-BO parameters are shown in bold.

\begin{tabular}{cc}
\hline vdW parameters & Value \\
\hline$e_{\mathrm{vdW}}$ & $\mathbf{0 . 4 3 7 8}$ \\
$r_{\mathrm{vdw}}$ & 2.9238 \\
$\hat{r}_{\mathrm{vdw}}$ & $\mathbf{3 . 3 0 0}$ \\
\hline
\end{tabular}

b. The van der Waals parameters for C-C interactions. $\boldsymbol{e}_{\boldsymbol{v} \boldsymbol{d} \boldsymbol{W}}$ is the van der Waals binding energy, $\boldsymbol{r}_{\boldsymbol{v} \boldsymbol{d} \boldsymbol{w}}$ is the position of the van der Waals minimum, and $\hat{r}_{v d w}$ is the radial cutoff used in calculating the counting factors. Refer to Mun et al [80] for the equations. Changes to the original MEAM-BO parameters are shown in bold. 
We recalibrated $\beta_{\text {bond }}^{(1)}, \beta_{\text {bond }}^{(3)}$, and $P_{\text {bond }}^{(3)}$ by fitting the FP results of the rotational energy barrier of $\mathrm{a} \mathrm{C}_{2} \mathrm{H}_{4}$ molecule, elastic constants for SWCNT, folding energy of CNT, RBMs of different SWCNTs. Note the changes in these parameters only affect the rotational energy barrier of the $\mathrm{C}_{2} \mathrm{H}_{4}$ molecule of the previous work ${ }^{79}$. Also, we corrected the $\theta_{S_{i 1 i 2}}$ parameter, a threshold value between 0 and 1, in conjunction with $S_{i 1 i 2}$ in Eq. (2.6) to select all pairs of atoms that form unsaturated $\mathrm{C}-\mathrm{C}$ bonds. The original value 0.5 has been changed to 0.001 , meaning more atom pairs are considered as unsaturated bonds. The change in $S_{i 1 i 2}$ leads to a change in the vdW parameters shown in the following Table (2.1), which does not show much change in the calculation results. In fact, the decreased threshold with the recalibrated vdW terms improved the elastic constants of graphite (see Supporting document). The parameters used in the MEAM-BO potential for the SWCNT are given in Table (2.1.a) and Table (2.1.b). The rest of the MEAMBO parameters remained the same as in Mun et al. ${ }^{79}$

\subsubsection{Parametrization procedure}

The workflow of parametrization and validating a new MEAM-BO* potential using interactive software on desktop computers, or non-interactive software on parallel clusters is illustrated in Figures (2.1) and (2.2). The interactive MEAM-BO graphical user interface (MEAMBO_GUI) and non-interactive script for MEAM-BO parameter study are written in MATLAB ${ }^{92}$. In this work, three parameters $\left(\boldsymbol{p}_{\mathbf{2}}^{(\mathbf{3})}, \boldsymbol{\beta}_{\mathbf{2}}^{(\mathbf{1})}, \boldsymbol{\beta}_{\mathbf{2}}^{(\mathbf{3})}\right)$. were chosen for the non-interactive study as these only affect the properties related to unsaturated bond cases. The minimum value, 
step size, and maximum value, of each parameter are: $\boldsymbol{p}_{2}^{(\mathbf{3})}=[0.1,50,5], \boldsymbol{\beta}_{2}^{(\mathbf{1})}=[0.1,50,5]$, $\boldsymbol{\beta}_{2}^{(3)}=[0.1,50,5]$. The RBM, $\left(\partial^{2} E / \partial^{2} \varepsilon\right), \mathrm{Y}$ and $(\Delta \mathrm{E})$ were calculated for all combinations of parameter values on a parallel cluster, and the results were post-processed to identify which combination of parameters values gave the least value of total root mean square error (RMSE). Table (2.4) compares the best RMSE of MEAM-BO* potential with those of original MEAM-BO, REBO, and ReaxFF 


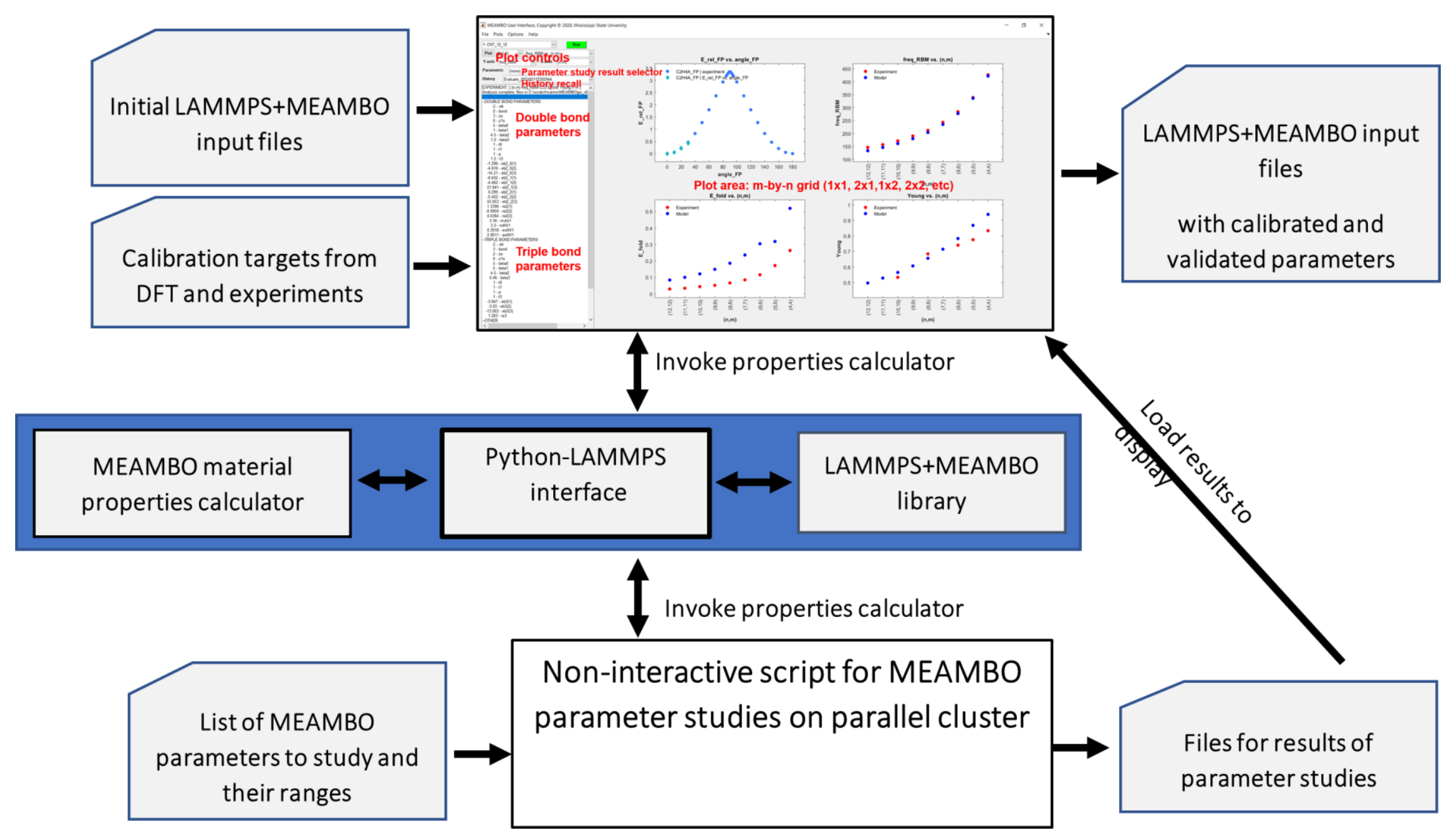

Figure 1.2 Interactive MEAM-BO Graphical 
File Plos Options Help

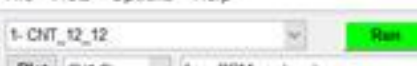

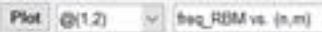

Yaxis free sed o Xaxis $(\mathrm{nm})$

Parametic (mones)

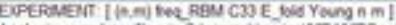

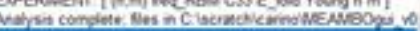

OOXRE BOTD PRAMETERS

C.
2. boed
3.25

3.

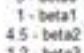

12- 10

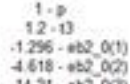

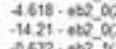

$462.62-1$

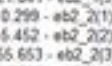

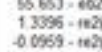

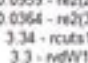

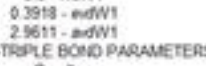

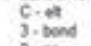

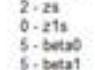

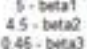

in

i.

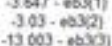

$(1200 \cdot 600$
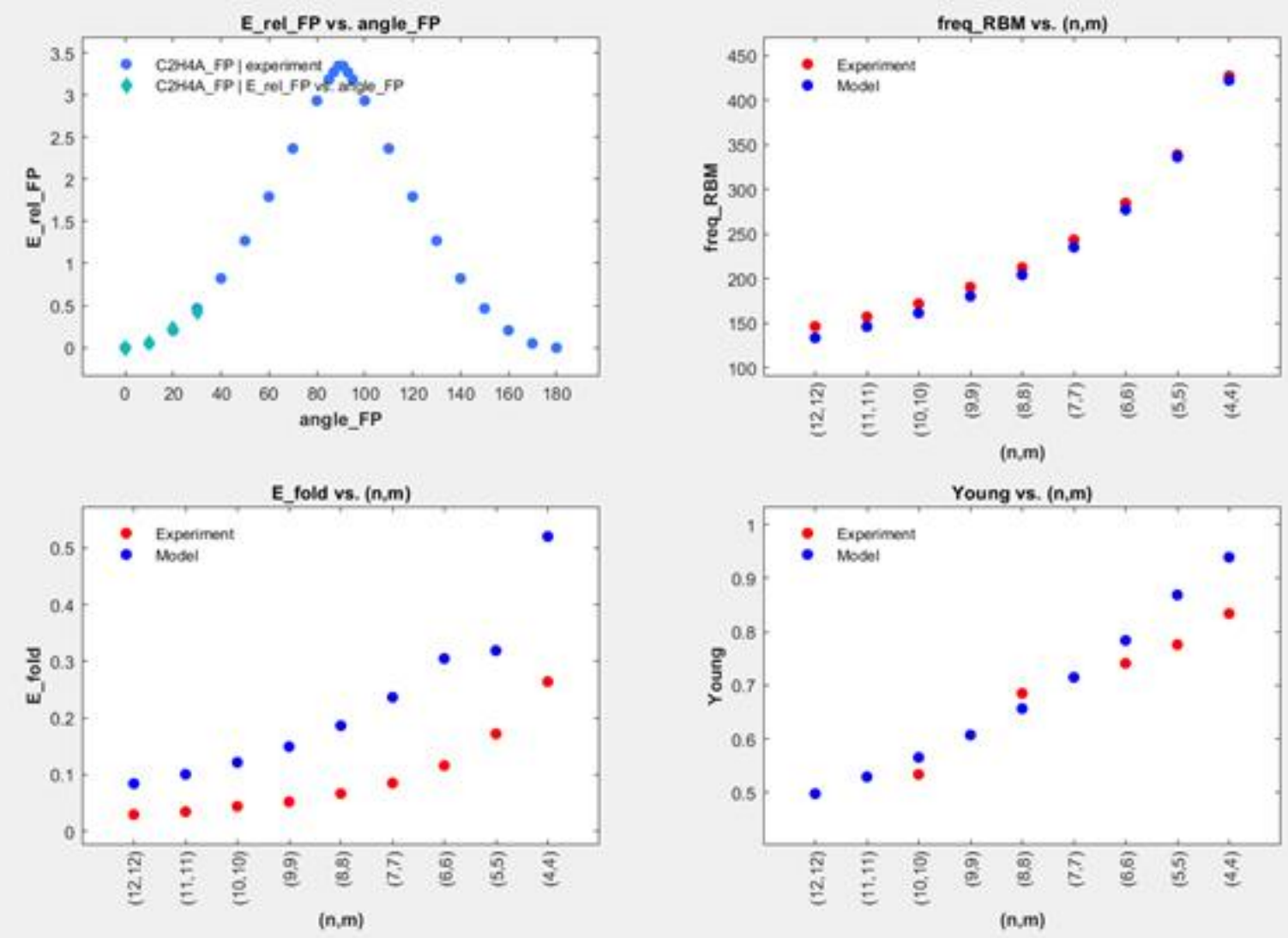

Figure 1.3 Screenshot for MEAM-BO user interface tools 


\subsubsection{Simulation Details}

We generated different carbon nanotubes with varying diameters by expressing the diameter of SWCNTs as a function of the $(n, m)$ chiral indices. $(n, m)$ is the conventional way of specifying a nanotube, showing how to form a nanotube from flat graphene ${ }^{38,70}$. It is prudent to define the diameter as $d=a_{0} \sqrt{3} / \pi \sqrt{n^{2}+n m+m^{2}}$, where $a_{0}=1.42 \AA$ is the first closest neighbor distance on the tube's surface and is the $\mathrm{C}-\mathrm{C}$ bond length. All computational cells are with periodic boundary conditions.

To calculate the RBM wavenumber, the SWCNT was relaxed to find the minimum energy conformation by a conjugate gradient mothed. Each atom was displaced $0.2 \AA$ from its equilibrium at $0 \mathrm{~K}$. We used the "Jazz" python script, ${ }^{93}$ which displaces each atom and calls LAMMPS ${ }^{94}$ to get the resulting forces and Hessian matrix. A Hessian matrix is the second derivative matrix of the energy with respect to displacements of all atoms in $x, y$, and $z$ directions expressed as Cartesian displacements as described in the following equation,

$$
H_{i, j}=\frac{\partial^{2} E}{\partial x_{i} \partial x_{j}}
$$

Afterward, we obtained the force constants by diagonalizing the Hessian matrix. We then calculated the vibrational frequencies using a weighted force matrix for the isotopic masses. The Hessian matrix has units of $\left(\mathrm{Kcal} / \mathrm{mol} . \AA^{2}\right)$ for the ReaxFF potential and units of $\left(\mathrm{eV} / \AA^{2}\right)$ for the MEAM-BO and REBO potentials.

When the mass-weighted Hessian was diagonalized, the result was a set of eigenvalues, $\varepsilon$ i, and associated eigenvectors, $\psi_{\mathrm{i}}$. The eigenvectors are the normal modes of vibration and form an orthonormal set. The eigenvalues represent the force constants for the modes divided by the effective mass with atomic mass units (u) as in the following equation, 


$$
\varepsilon_{i}=k_{i} / \mu_{i}
$$

where eigenvalues, $\varepsilon_{\mathrm{i}}$, of the mass-weighted Hessian are related to the force-constant, $k_{\mathrm{i}}$, and reduced mass, $\mu_{\mathrm{i}}$ with units $\left(\mathrm{kcal} / \mathrm{mol} . \mathrm{Amu} . \AA^{2}\right)$ for ReaxFF and $\left(e V / A m u . \AA^{2}\right)$ for MEAM-BO and REBO. The eigenvalues were then converted into wavenumbers, $v_{\mathrm{i}}$, in $\mathrm{cm}^{-1}$ via

$$
v_{i}=\frac{\varepsilon_{i}^{1 / 2}}{2 \pi c}
$$

where $c$ is the speed of light in centimeters per second.

To identify the vibrational mode of a SWCNT that corresponds to the RBM, we analyzed the eigenvectors. Changes in the axial lengths are not involved in the RBM vibration modes, only symmetric deformations in $x$ and $y$. Among the candidates, we interpret the symmetric condition to be when eigenvectors are pointing at the same direction as the coordinate vectors (i.e. atomic positions). We then normalized the coordinates and the eigenvectors to get the average difference between them. We measured that average difference between the eigenvectors and the coordinates and determined RBM to be the smallest difference value.

For the elastic properties, we calculated the tensile strength by applying various strains ranging from $-2 \%$ to $+2 \%$ in the $z$ axial direction. We then calculated the total energy for each configuration. Also, we obtained the elastic constants using quadratic functions to fit the resultant curves. Young's modulus was calculated using the standard bulk definition for each SWCNT diameter employing the potentials of REBO, ReaxFF, and MEAM-BO. 


\subsection{Results}

\subsubsection{Radial Breathing Modes (RBMs)}

We performed simulations for infinite armchair SWCNTs with radii ranging from 2.71 to $8.13 \AA$ and atom numbers between 80 and 240 atoms. The amplitude of the radial oscillations incurred a limited displacement, usually less than $0.5 \%{ }^{52}$ to get a pure RBM. The RBM wavenumber of our calculation from MEAM-BO, MEAM-BO*, ReaxFF, and REBO potentials are shown in Table (2.2). Figure (2.3) shows the RBM modes versus the SWCNT diameter and the inverse of the diameter. 
Table 1.2 Radial Breathing Mode results

\begin{tabular}{|c|c|c|c|c|c|c|c|c|c|c|}
\hline$(n, n)$ & d $(\AA)$ & $\begin{array}{l}\text { Experiments } \\
{[30,32]}\end{array}$ & & $\begin{array}{l}\text { DFT } \\
{[38,39,40}\end{array}$ & & & MEAM-BO & MEAM-BO* & REBO & ReaxFF \\
\hline$(12,12)$ & 16.2 & 147 & & 146 & - & - & 133.2 & 137.7 & 134.4 & 142.8 \\
\hline$(11,11)$ & 14.8 & 162 & 150 & 159 & 158 & - & 145.7 & 150.6 & 146.8 & 155.5 \\
\hline$(10,10)$ & 13.4 & 177 & 165 & 175 & 173.6 & 169 & 160.9 & 166.2 & 161.6 & 170.6 \\
\hline$(9,9)$ & 12.2 & 195 & 183 & 195 & 192.7 & 187 & 179.6 & 185.4 & 179.8 & 188.9 \\
\hline$(\mathbf{8 , 8})$ & 10.8 & 211 & 206 & 219 & 216.5 & 210 & 203.4 & 209.8 & 202.6 & 211.4 \\
\hline$(7,7)$ & 9.5 & & & & 246.2 & 241 & 234.5 & 241.9 & 232 & 239.4 \\
\hline$(6,6)$ & 8.1 & & & & 284.3 & 285 & 276.6 & 286 & 272.4 & 275.1 \\
\hline$(5,5)$ & 6.8 & & & & 337.3 & 341 & 335.6 & 349.9 & 330 & 325 \\
\hline$(4,4)$ & 5.4 & & & & 424.3 & 430 & 421.6 & 448.8 & 418.4 & 544.4 \\
\hline
\end{tabular}

Radial breathing mode frequency results for the various armchair nanotubes. RBM wavenumber results are presented in units of $\mathrm{cm}^{-1}$. The results were obtained by using the reaction force fields original MEAM-BO, updated MEAM-BO*, ReaxFF, and REBO and comparing them with experiments and DFT calculations based on the Local-Density Approximation (LDA) data. 

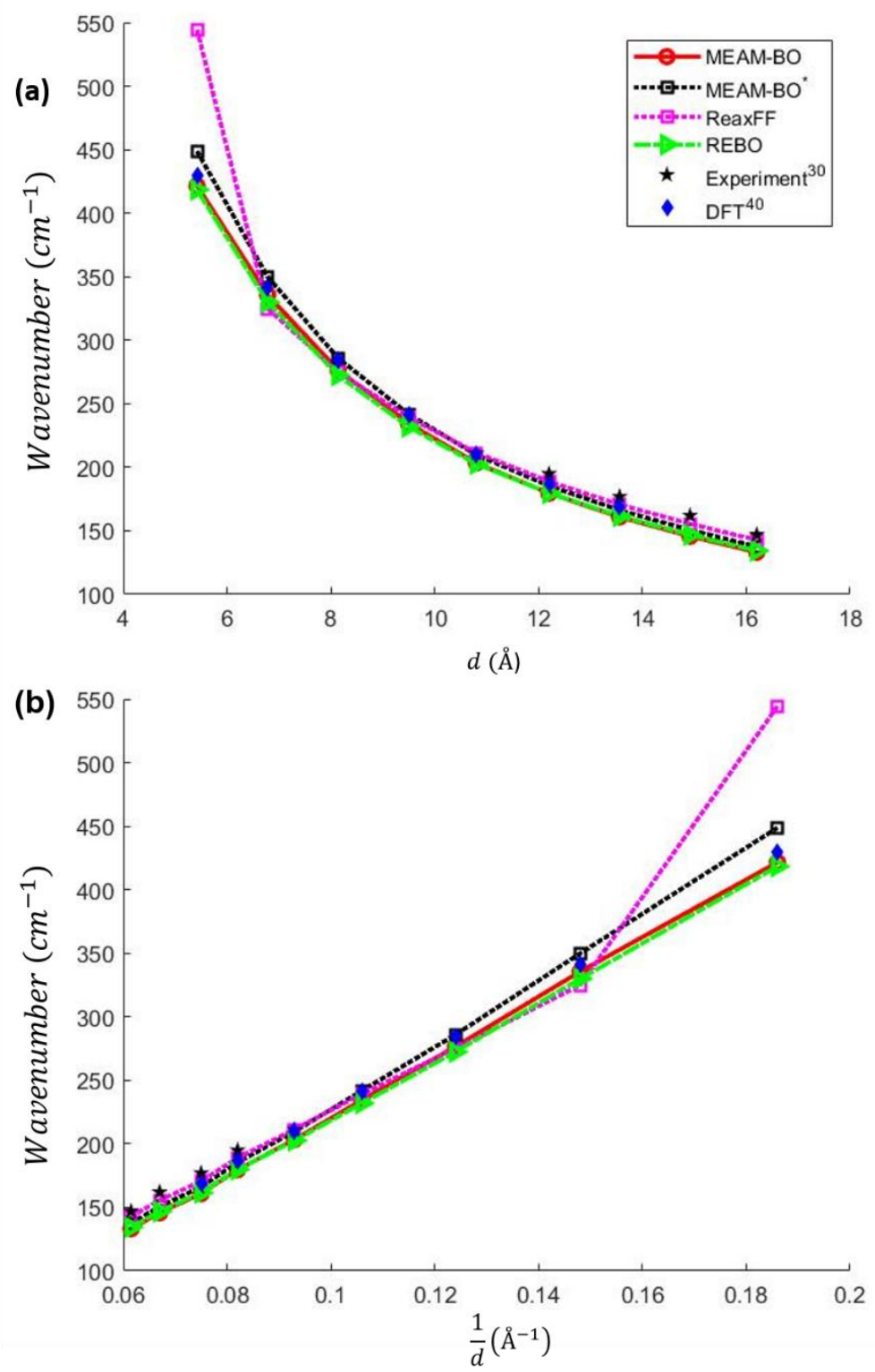

Figure 1.4 Plot of radial breathing mode result

Breathing mode wavenumber as a function of (a) the carbon nanotube diameter and (b) the inverse diameter. MEAM-BO, MEAM-BO*, ReaxFF, and REBO results are compared to the experiments of Kuzmany et al. [30] (black pentagram) and the DFT calculations of Lawler et al. [40] (blue diamond). 


\subsubsection{Young's Modulus}

An important attribute of the RBM and axial deformation is Young's modulus. However, a significant disparity in Young's modulus values of SWCNTs is present in the literature due to researchers using different definitions ${ }^{47}$ and different methods of determination. We present the elastic properties of armchair nanotubes by using the second derivative of the energy per atom with respect to the axial strain $\partial^{2} E / \partial^{2} \varepsilon$ at zero $\mathrm{K}$. The findings are as presented in Table (2.3) and Figure (2.5). As expected for the potentials used herein, as the diameter increased, the second derivative of the energy per atom decreased until saturating at a particular large diameter.

Therefore, we can compare these results for $\left(\partial^{2} E / \partial^{2} \varepsilon\right)$ in infinite diameter to the tensile strength for a graphene sheet or to graphite's elastic constant if we ignore interactions between the layers ${ }^{95}$. We also calculated the Young's modulus versus SWCNT diameter size related to the second derivative of the strain energy found earlier. In a standard bulk elastic calculation, the conventional formula for Youngs's modulus is given as

$$
Y=\left.\frac{1}{V_{0}} \frac{\partial^{2} E}{\partial^{2} \varepsilon}\right|_{\varepsilon=0}
$$

In Eq. (2.11), $V_{0}$ is the effective volume at equilibrium, and $E$ is the strain energy.

In the instance where there is a single nanotube, one must adopt a suitable convention that defines $V_{0}$. The volume of a single-wall carbon nanotube is the cylindrical volume $V_{C}$ with the half effective SWCNT diameter $\left(d_{e}\right)$ and a representative volume element per unit length $^{96}\left(\zeta_{l}=\frac{4}{3 a_{0}} \sqrt{n^{2}+m^{2}+m n}\right)$ with units atom/Å. The effective diameter $\left(d_{e}\right)$ is equal to 
the diameter of the SWCNT $(d)$, includes the van der Waals equilibrium separation distance $(h)$ which is the thickness of the nanotube (Fig.2.4).

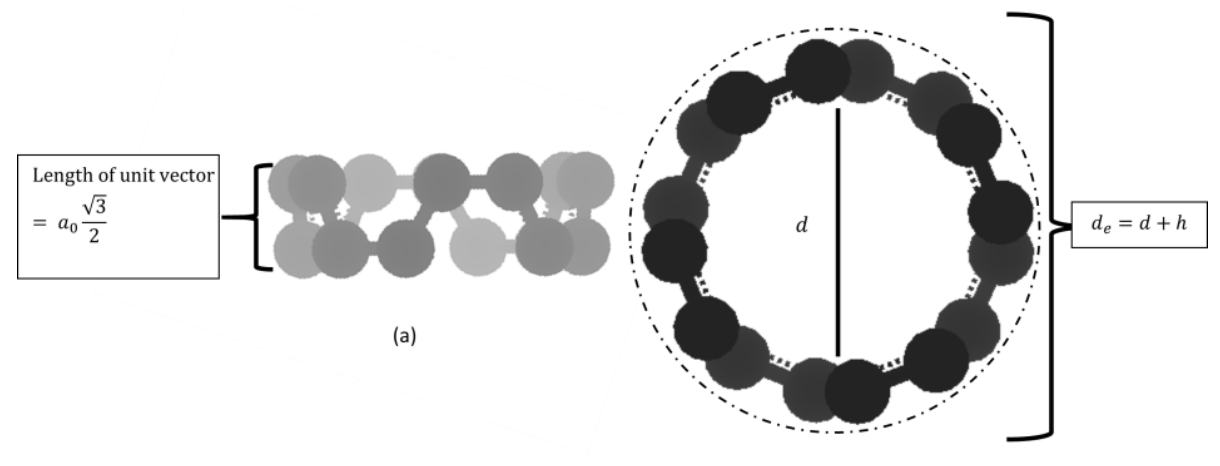

(b)

Figure 1.5 Schematic of carbon nanotube

a) Front schematic of carbon nanotube, and (b) Top view of carbon nanotube with diameter d and thickness $(h)$.

Several investigators have taken the SWCNT equilibrium separation distance to be equal to that of the equilibrium separation distance of graphene sheets of $3.4 \AA$. However, we use the definition whereby we take the single-wall nanotubes to form an enclosed hexagonally packed structure with the constant volume fraction of the identical nanotubes hexagonal close packing $\left(\eta_{a}=\frac{\pi}{2 \sqrt{3}}\right)$. As such, the effective volume of the unit cell per atom is given by the formula

$$
\begin{array}{r}
V_{0}=\frac{V_{C}}{\eta_{a}}, \\
V_{0}=\frac{3 a_{0}(d+3.4)^{2}}{2 N}
\end{array}
$$


where the total number of atoms in $(\mathrm{n}, \mathrm{n})$ armchair nanotube is $N=4 n$. Accordingly, Young's modulus will have an inverse quadratic relationship with the diameter as given in the following equation,

$$
Y=\left.\left(\frac{2 N}{3 a_{0}(d+3.4)^{2}}\right) \cdot \frac{\partial^{2} E}{\partial^{2} \varepsilon}\right|_{\varepsilon=0}
$$

Figure (2.5) shows Young's modulus relationship with the nanotube diameter and the inverse square of the diameter from the MEAM-BO, MEAM-BO*, ReaxFF, and REBO results. We note that all of the potentials are fairly close to the DFT elastic modulus ${ }^{27}$ with MEAM-BO being the closest. The tight-binding calculation $(\mathrm{TB})^{69}$ values for the Young's modulus are much too large. They use the same thickness as we use, but they have a different definition of the volume as they treated the SWCNT as a hollow cylinder.

Table 1.3 The Second Derivative of the Energy Results

\begin{tabular}{c|ccccccc}
$(\mathbf{n , n})$ & $\mathbf{d}(\AA)$ & DFT [47] & DFT [95] & MEAM-BO & MEAM-BO* & ReaxFF & REBO \\
\hline $\mathbf{( 1 2 , 1 2})$ & 16.2 & & 65.7 & 52.9 & 57.1 & 65.4 & 61.5 \\
$(\mathbf{1 1 , 1 1})$ & 14.8 & & 62.4 & 53.3 & 57.2 & 65.3 & 61.5 \\
$(\mathbf{1 0 , 1 0})$ & 13.4 & 52 & 62.4 & 53.7 & 57.5 & 65.2 & 61.5 \\
$(\mathbf{9 , 9})$ & 12.2 & & 60.5 & 54.2 & 57.8 & 64.9 & 61.6 \\
$(\mathbf{8 , 8})$ & 10.8 & 59 & 59.6 & 55 & 58.3 & 64.6 & 61.6 \\
$(\mathbf{7 , 7})$ & 9.5 & & 59.5 & 56.1 & 59 & 63.9 & 61.6 \\
$(\mathbf{6 , 6})$ & 8.1 & 56 & 57.4 & 57.5 & 60.3 & 62.9 & 61.7 \\
$(\mathbf{5 , 5})$ & 6.8 & 55 & 57.7 & 59.2 & 62.2 & 62.6 & 61.8 \\
$(\mathbf{4 , 4})$ & 5.4 & 56 & 57.1 & 60.6 & 65.6 & 108.4 & 62 \\
\hline
\end{tabular}

The second derivative of the energy $\left(\partial^{2} E / \partial^{2} \varepsilon\right)(\mathrm{eV} /$ atoms). Calculations for different armchair nanotube diameters $(d)$ were obtained by using MEAM-BO, MEAM-BO*, ReaxFF, and REBO potentials and compared with Sánchez-Portal et al. [47] and Alford et al. [95] other DFT calculations. 


\subsubsection{Folding Energies}

We carried out folding energy calculations for different diameters of armchair nanotubes with the MEAM-BO, MEAM-BO*, REBO, and ReaxFF potentials. Nielson et $a l^{78}$ considered graphite as the reference structure and used it to measure the folding energy of nanotubes by using DFT calculations and the ReaxFF potential. In our work, we considered a graphene sheet as a reference to be more realistic. We determined the cohesive energy of a graphene sheet in MEAMBO, REBO, and ReaxFF potentials to be $-7.4696 \mathrm{eV} /$ atom $^{79},-7.4266 \mathrm{eV} / \mathrm{atom}$, and -7.5244 eV/atom, respectively.

Figure (2.6) depicts how the relative energy per carbon depends on the diameter of the armchair carbon nanotube as the inverse square of the diameter following continuum elasticity theory. The constants of proportionality for MEAM-BO, MEAM-BO*, REBO, and ReaxFF potentials were $C=17.36 \mathrm{eV}^{2}, C=7.679 \mathrm{eV}^{2}, C=4.83 \mathrm{eV}^{2}$, and $C=6.63 \mathrm{eV} \AA^{2}$, respectively. The folding energy in the calculation with MEAM-BO was slightly greater than predicted from the DFT calculations or the other potentials, but it did show a similar monotonically decreasing trend as the diameter increased. Furthermore, the MEAM-BO results showed a clear folding energy that increased faster when the diameter was less than $10 \AA$. However, using the updated MEAM-BO* yielded more accurate results that were the closest to the DFT calculations 

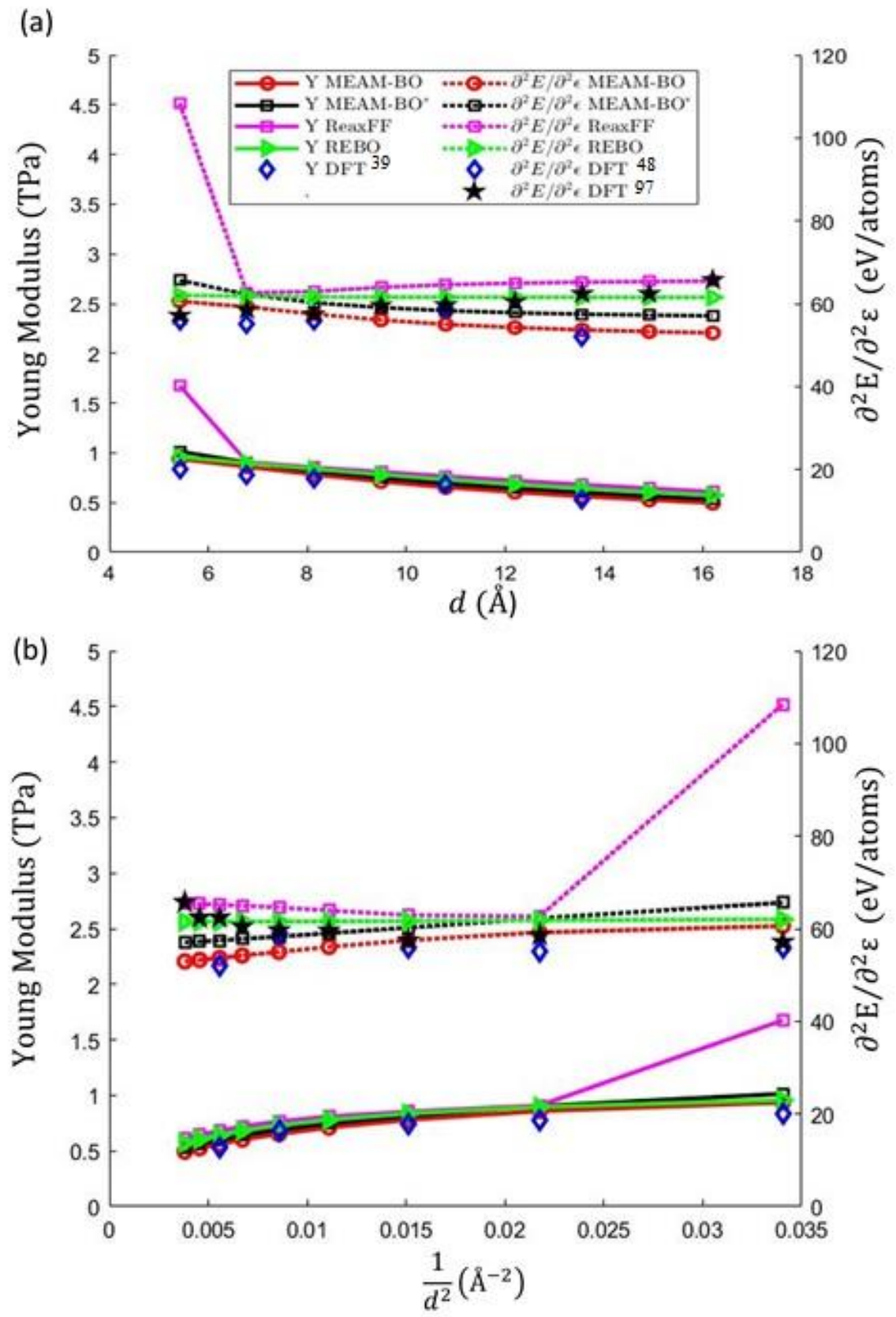

Figure 1.6 Plot of elastic properties results

a) Young's modulus $(Y)$ (solid lines) and second derivative of the energies $\left(\partial^{2} E / \partial^{2} \varepsilon\right)$ (dashed lines) calculations versus inverse diameters squared of the armchair nanotubes following a quadratic proportion fit.

b) Young's modulus $(Y)$ (solid lines) and second derivative of the energies $\left(\partial^{2} E / \partial^{2} \varepsilon\right)$ (dashed lines) calculations versus the armchair nanotube diameter from MEAM-BO, MEAM-BO*, ReaxFF, REBO, Sánchez-Portal et al.'s [47] (blue diamond), and Alford et al [95] (black star) work using DFT calculations 


\subsection{Discussion}

\subsubsection{Radial Breathing Modes (RBMs)}

As shown in previous studies ${ }^{30,31,37,40}$, the RBM frequency from our results were inversely proportional to the nanotube diameter $(d)$. Figures 2.3(a) and 2.3(b) show the RBM modes versus $d$ and $1 / d$, respectively. We found a linear correlation to the original MEAM-BO and updated MEAM-BO* data respectively with $A=2233 \AA \mathrm{cm}^{-1}$ and $A=2332 \AA \mathrm{cm}^{-1}$ by using. $A=2171 \AA$ $\mathrm{cm}^{-1}, B=3.1 \AA^{3} \mathrm{~cm}^{-1}$ and $A=2215 \AA \mathrm{cm}^{-1}, B=5.9 \AA^{3} \mathrm{~cm}^{-1}$ in Eq.(3.2). We note that both MEAMBO potentials and REBO are close to the DFT results; ${ }^{40}$ however, ReaxFF greatly increased from the others when the diameter was less than $7 \AA$ indicating a lesser accuracy and breakdown of model.

\subsubsection{Young's Modulus}

Because different researchers used different atomistic potentials, numerical methods, SWCNT diameters, and methods for determining the associated volume for quantifying Young's modulus for SWCNTs, a range from 0.27 to $5.5 \mathrm{TPa}$ has arisen in the literature. ${ }^{27,49,50,61,82}$ Our results gave two main trends for Young's modulus: (1) a definite correlation as the SWCNT diameter decreased the elastic modulus linearly increased, and (2) the MEAM-BO, MEAM-BO*, ReaxFF, REBO potentials gave very close results to each other and to the DFT results ${ }^{27}$ as shown in Figure (2.5). 

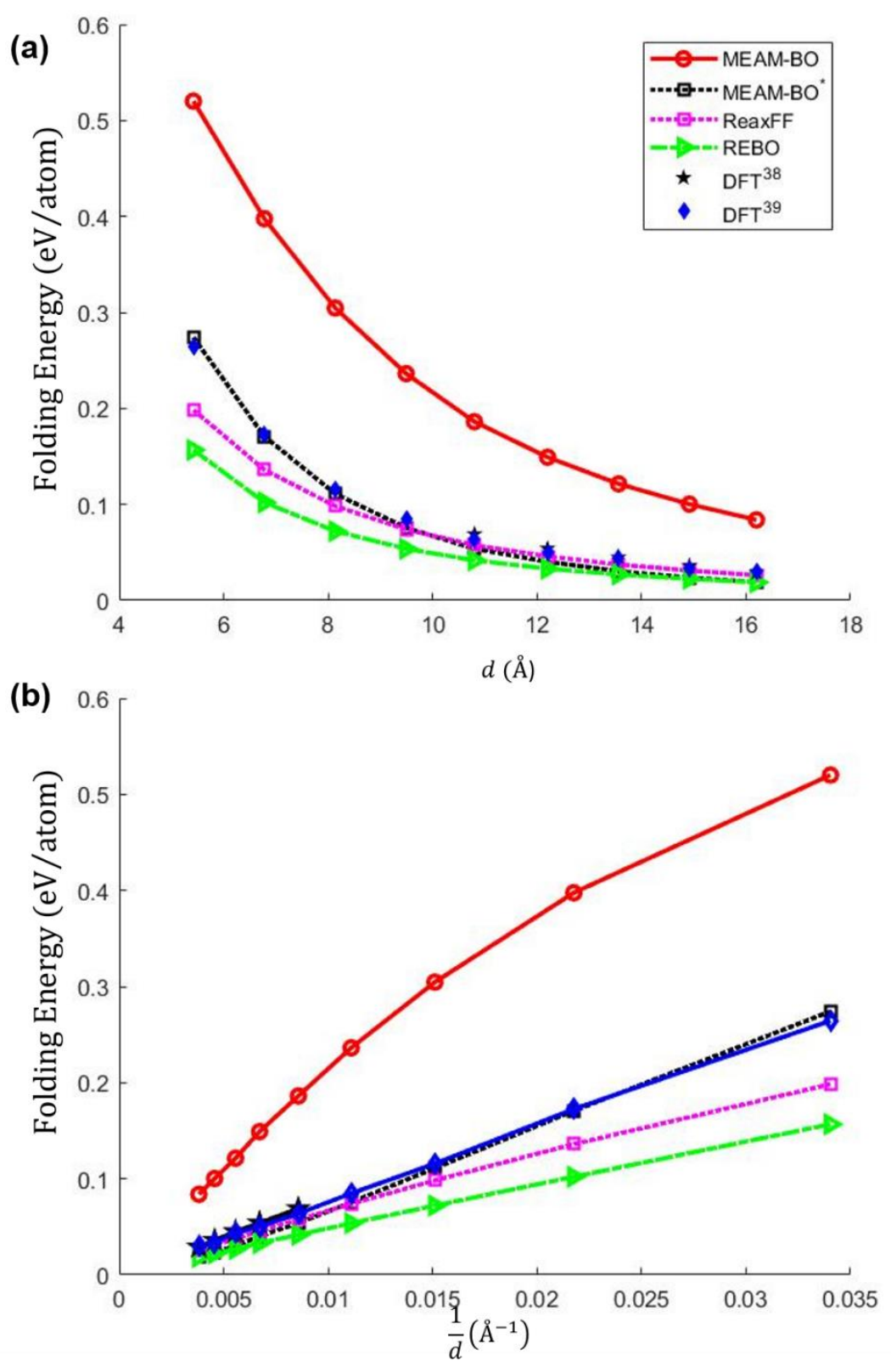

Figure 1.7 Plot of folding energy results

Folding energy results for armchair nanotubes as a function of a) the dimeter. b) the inverse diameter squared. For MEAM-BO, MEAM-BO*, ReaxFF, REBO calculations, and DFT calculations $[38,39]$.

The wall thickness of a SWCNT is not well defined and as such has led to different elastic moduli in the literature. Continuum elasticity theory ${ }^{82}$ argues that Young's modulus 
should be dependent upon the nanotube's wall thickness. Yakobson et al. ${ }^{34}$ garnered an exceedingly large Young's modulus with a very small wall thickness of $0.6 \AA$. Alternatively, Kalamkarov et $a l .{ }^{83}$ and Halicioglu ${ }^{84}$ used a wall thickness of $6.8 \AA$. These wall thickness variations gave values of Young's modulus ranging from $0.27 \mathrm{TPa}$ to $5.5 \mathrm{TPa}$. Like many other studies, we employed a SWCNT wall thickness of $3.4 \AA$.

In our work, MEAM-BO and MEAM-BO* gave the closest Young's modulus agreement for the smaller nanotube diameters, but REBO agreed better for larger nanotube diameters. Another contrast between the potentials' results related to the second derivative of the energies $\left(\partial^{2} E / \partial^{2} \varepsilon\right)$, we showed that the $\left(\partial^{2} E / \partial^{2} \varepsilon\right)$ values from the REBO and ReaxFF changed very little with tube diameter, while MEAM-BO and MEAM-BO* changed according to continuum elasticity theory.

\subsubsection{Folding Energy}

The folding energy in the calculation of MEAM-BO was slightly greater than predicted from the DFT calculations or the other potentials, but it did show a similar monotonically decreasing trend as the diameter increased. The updated MEAM-BO* shows improvement in the results and the greatest agreement with DFT calculations. We find the constant of proportionality in MEAM-BO* following Eq. (2.3) as $C_{1}=5.32 \mathrm{eV} / \AA^{2}, C_{2}=29.15 \mathrm{eV} / \AA^{4}$

\subsubsection{Accuracy of MD Simulation}

In table (2.4), we calculate the root mean square error (RMSE) of the Radial Breathing Mode $(\mathrm{RBM})$, second derivative $\left(\partial^{2} E / \partial^{2} \varepsilon\right)$, Young modulus $(Y)$, and folding energy $(\Delta \mathrm{E})$ for each potential. The ReaxFF potential has a higher value of the RMSE, while all the rest of the 
potentials have a value around 5. The updated MEAM-BO potential, MEAM-BO*, improved results for both the folding energy and RBM.

Table 1.4 Root Mean Square Error (RMSE) of all the calculation for MEAM-BO, MEAMBO*, REBO, and ReaxFF potentials.

\begin{tabular}{l|llll} 
& $\begin{array}{l}\text { MEAM-BO } \\
\text { (RMSE) }\end{array}$ & $\begin{array}{l}\text { MEAM-BO* } \\
\text { (RMSE) }\end{array}$ & $\begin{array}{l}\text { REBO } \\
\text { (RMSE) }\end{array}$ & $\begin{array}{l}\text { ReaxFF } \\
\text { (RMSE) }\end{array}$ \\
\hline RBM & 9.6 & 9.3 & 9.7 & 33.3 \\
\multicolumn{1}{c|}{$\left(\boldsymbol{\partial}^{2} \boldsymbol{E} / \boldsymbol{\partial}^{2} \boldsymbol{\varepsilon}\right)$} & 3.7 & 4.5 & 3.2 & 14.6 \\
$\mathbf{Y}$ & 0.06 & 0.11 & 0.04 & 0.3 \\
$\boldsymbol{\Delta} \mathbf{E}$ & 0.153 & 0.0098 & 0.048 & 0.0290 \\
\hline Total RMSE (\%) & 5.44 & 5.47 & 5.47 & 19.45 \\
\hline
\end{tabular}

\subsubsection{Root Mean Square Error (RMSE) results of all the calculations.}

We demonstrated that the MEAM-BO potential works very well for single-wall carbon nanotubes (SWCNTs) with different diameters. Additionally, the MEAM-BO results were validated by other atomistic potentials, DFT, and physical experiments. The present study provides three clear conclusions.

First, the MEAM-BO and MEAM-BO* potentials results agreed with results from previous atomistic studies as the nanotube diameter $(d)$ inversely affected the RBM. We also noted that the both MEAM-BO's and REBO results were closer to the DFT results compared to the ReaxFF results.

Second, MEAM-BO's excellent agreement with the experimental and DFT RBM wavenumbers suggests that MEAM-BO is great at capturing the unsaturated hydrocarbons' curvature effects. The curvature is associated with the second derivative of the energy potential. MEAM-BO showed the gradual increase of the second derivative of the energies when the diameter was more than another reactive force fields, as did the experiments and DFT calculations. 
The curvature is also related to the elastic moduli, and Young's modulus from MEAM-BO was closer to the DFT results than REBO and ReaxFF.

Third, the folding energy in the calculation of MEAM-BO was greater than that from the DFT calculations and from the other potentials, but a similar trend existed in that as the diameter increased the folding energy monotonically decreased. In addition, MEAM-BO* improved the calculation results of the folding energy and appear to be in accord with results with DFT calculations. 


\section{CHAPTER III}

\section{MECHANICAL PROPERTIES OF DIFFERENT PARTIALLY UNZIPPED CARBON NANOTUBES BY ATOMISTIC SIMULATION}

\subsection{Introduction}

Creating graphene nanosheets has one preferable method by using cutting techniques that are applied to carbon nanotubes in order to achieve graphene nanoribbons (GNRs). This potential industrial-scale method for producing GNRs that can be used in an electronic device comes from the process of unzipping CNTs. As a result, a large amount of research has focused on discovering the properties of combining CNTs with GNRs because there are excellent properties that can be utilized across mechanical, electronic, and magnetic applications. Moreover, unzipped carbon nanotubes may be able to enhance the strength of the interface structure of composites ${ }^{97,98}$. Experimentally, unzipping carbon nanotubes can take place through oxidization ${ }^{99}$, electrochemical anodic oxidation, intercalation ${ }^{100}$, plasma etching ${ }^{101}$, or functionalization ${ }^{102}$ using a scanningtunneling-microscope (STM) manipulation. Cano Marquez et al. ${ }^{103}$ also examined multi-walled carbon nanotubes (MWNT) and found that through intercalation of ammonia and lithium, followed by exfoliation, the tubes could be opened. The best results were found when intercalation was conducted through the use of an acid treatment and abrupt heating after the treatment. This resulted in multilayered flat structures of a graphitic nature, or nanoribbons, MWNTs that were partially open, and graphene flakes. Rangel et al. ${ }^{104}$ studied the unzipping process of carbon nanotubes with potassium permanganate by ab initio density functional theory calculations. They found that the 
potassium permanganate attacks one of the internal $\mathrm{C}-\mathrm{C}$ bonds of the $\mathrm{CNT}$, then stretches and breaks it as the first step of unzipping a CNT. That defect then creates weakness along the length of the CNT, causing it to unzip easily.

In a theoretical study, Huang et al. ${ }^{105}$ reported that partially unzipped carbon nanotubes (PUCNTS) have valuable electronic and magnetic properties that are dependent on the length of their opening. Costamagna et al. ${ }^{106}$ further studied the properties of PUCNTs by applying an external magnetic field parallel to a long PUCNT, and found that the magnetic field only affected the electronic state in the CNT region. Additionally, Chen et al. ${ }^{107}$ revealed that the thermal conductance of PUCNTs is dependent on the width and length of the unzipped part. In molecular dynamics simulations, Tang et al. ${ }^{108}$ explored the stability of PUCNTS with temperature and highlighted that different structures of unzipped carbon nanotubes tend to selfheal with rising temperatures and when the edge dangling bonds are not passivated. Also, the unzipped graphene ends tend to wrap up and form various patterns in the case of a large length cut or a small diameter. The thermal conductivity of PUCNTs was investigated by Ni et al. ${ }^{109}$ who, using classical non-equilibrium MD, suggested that partially unzipped carbon nanotubes may have the potential to aid in thermal management. Lastly, Novotny and Novotný ${ }^{110}$ predicted using a simple tight-binding model that combinations of partially unzipped carbon nanotubes together with graphene nanoribbons could form 'quantum dragons' wherein even strongly disordered system can have extremely small electrical resistance and perhaps other novel electronic and magnetic properties. The unzipped carbon nanotubes have different mechanical properties. For example, they have high thermal conductivity with very high tensile strength and large elongation which means that they are very elastic. They also have different aspect ratios 
and are highly flexible at different bend levels. This is due to their low thermal expansion coefficient $^{111}$

In this work, we built five different structures of partially unzipped carbon nanotubes with one or two nanoribbons that have edge dangling bonds which are not passivated to explore their structural stability and mechanical properties. We subsequently performed force field $\mathrm{REBO}^{112}$-based molecular dynamics simulations on these structures.

\subsection{Methods}

Using Visual Molecular Dynamics (VMD) ${ }^{113}$, as well as Materials and Processes Simulation (MAPS) $)^{114}$, it was possible to build $(10,10)$ single wall Carbon nanotubes (SWCNT) with length 400 Å configuration. This structure was then partially unzipped in five different ways with the same length of cutting of $240 \AA$ except the $1^{\text {st }}$ structure, which was cut to the end with the length of $252 \AA$. The five structures are shown in Figure.3.1 as built systems.

- $\left.1^{\text {st }}\right)$ a longitudinally formed nanoribbon at the end.

- $\left.\quad 2^{\text {nd }}\right)$ a longitudinally formed nanoribbon between normal nanotube and one-sideinverted nanotube, in which the inside became outside on one end of the tube

- $3^{\text {rd }}$ two longitudinal nanoribbons between normal nanotubes.

- $4^{\text {th }}$ ) two longitudinal nanoribbons between normal nanotube and one-side-inverted nanotube, in which the inside became outside on one end of the tube

- $\left.5^{\text {th }}\right)$ two twist nanoribbons of length between normal nanotubes.

The experiment required a cutting method so that five different partially unzipped CNT structures could be obtained longitudinally as they were cut with one row of $\mathrm{C}-\mathrm{C}$ bonds. It was observed that edge atoms had dangling bond structures within all the structures that were created, because none had any hydrogen passivation. This leads to exciting new phenomenon. 
After the initial construction of the unzipped configurations were built, the models were then minimized using the reactive force fields REBO within the LAMMPS software. At this point, the REBO potentials were allowed to equilibrate with $0.4 \mathrm{fs}$ time steps that were provided through a NVT dynamics at a temperature of $300 \mathrm{~K}$. This created a condition in which the temperature, pressure, density, and total energy of the system could fluctuate about a relatively constant value. This provided for stabilization of the energy within the system.

We then heated the systems at different temperatures equilibrated by following the NVT ensemble using the Nose-Hoover thermostat in 3.5 ns. Non-periodic models were replicated, and we used the conditions shown in Figure 3.2. for the purpose of applied tension after the equilibration stage. We held the hydrogen atoms in the bottom of the nanotubes fixed, and moved the upper group that includes the hydrogen atoms on the top and the first ring of the nanotube by a constant velocity of $0.3 \mathrm{~nm} / \mathrm{ps}$ along the tube direction.

For the purpose of obtaining stress-strain response of the partially unzipped CNTs during uniaxial tension, the stress was calculated using the following equation ${ }^{115}$ :

$$
v_{z}=\frac{\sum_{K}^{N} m_{k} v_{z k}}{V}+\frac{\sum_{K}^{N} f_{z k} r_{z k}}{V}
$$

Here $N$ is the number of the atoms in the system, $v_{z k}$ is the $\mathrm{z}$ component of the velocity of the $\mathrm{k}^{\text {th }}$ atom, $f_{z k}$ is the $\mathrm{z}$ component of the force applied on the $\mathrm{k}^{\text {th }}$ atom, $r_{z k}$ is the $\mathrm{z}$ component of position vector of the $\mathrm{k}^{\text {th }}$ atom, and $V$ is the volume of the model. We consider the volume in our systems of PUCNT same as a completed zipped CNT which following this formula.

$$
V=2 \pi R h L
$$

Where R is the radius of CNT $(10,10), h$ is the tube's wall thickness ( $3.5 \AA)$, and L is the length of the tube. 


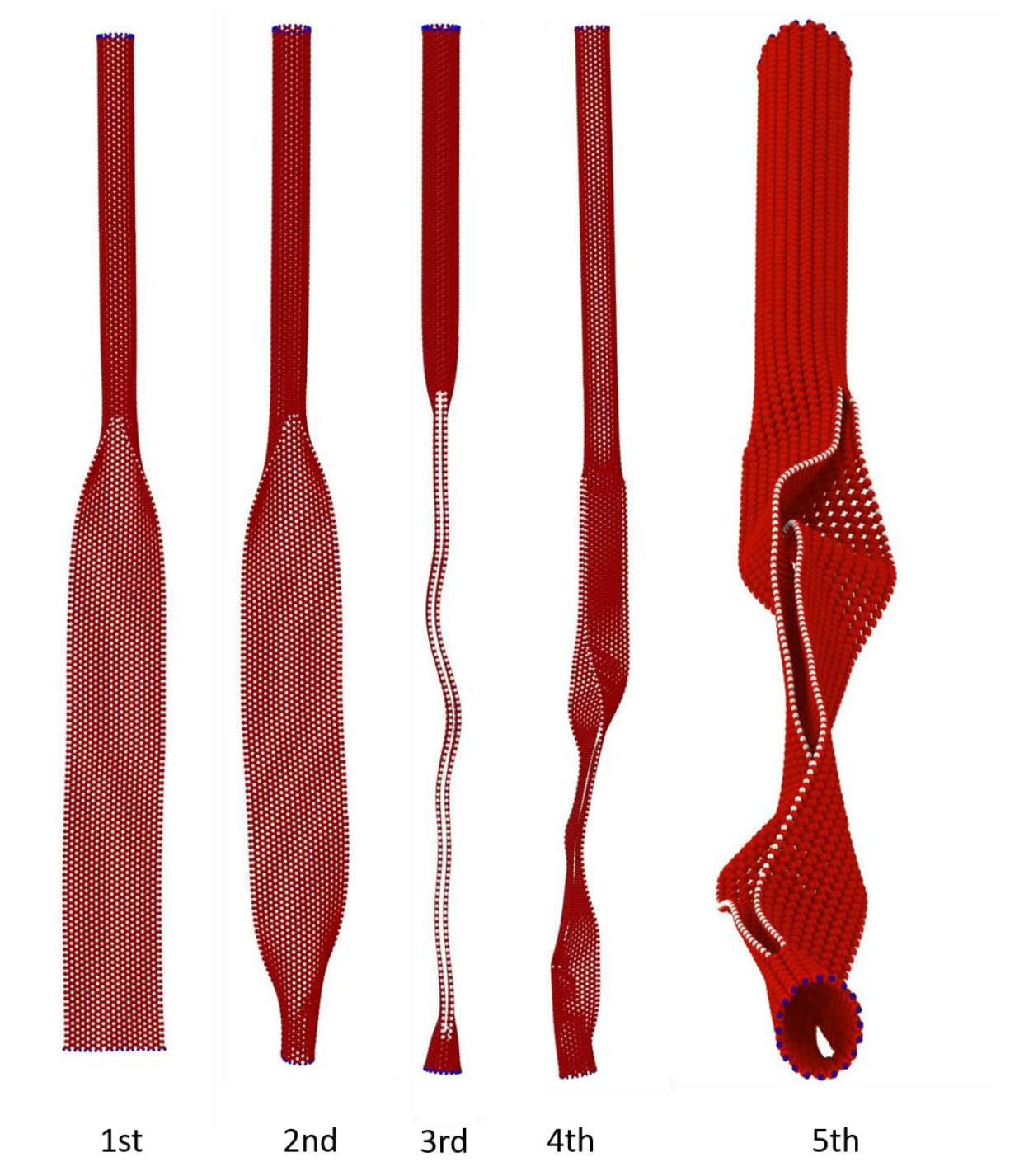

Figure 1.8 Schematic of five partially unzipped carbon nanotube (PUCNT). 


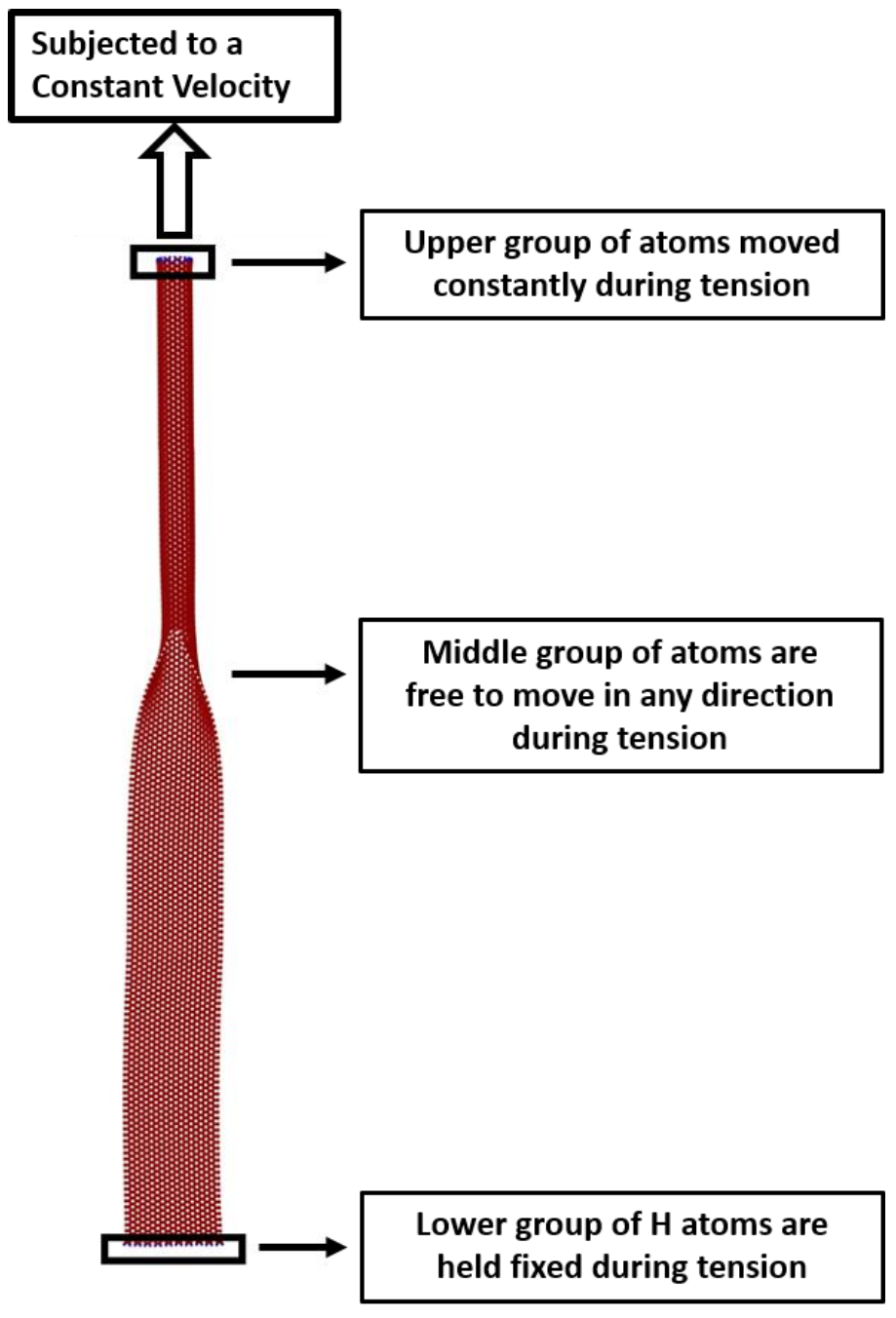

Figure 1.9 Molecular dynamics (MD) simulation set-up for the unzipped CNT under uniaxial deformation in the $\mathrm{z}$ direction (vertical direction).

\subsection{Results and Discussions}

\subsubsection{Self -Healing}

\subsubsection{Temperature}

From the analysis of Figure 3.3, the partially unzipped carbon nanotubes (PUCNT) depict changes in temperature with the highest at 75 with total energy changes at different times. With a cut length of $L=70(\AA)$ of the partially unzipped $(12,12) \mathrm{CNT}$, a MD simulation is performed 
at different temperatures of $400 \mathrm{~K}, 600 \mathrm{~K}$, and $1000 \mathrm{~K}$. This means that the unzipped length of the nano-tube decreases during the simulation time of nanoseconds at these different temperatures. The total energy is different, being a function of time and temperature.

Accordingly, the partially unzipped CNT depicted here exhibits self-healing effects based on the curve provided. The change in time and temperature provide a change in length of the unzipped portion on nano-second timescales, which confirms the nano-tubes self-healing property. The CNT $(12,12)$ at $1000 \mathrm{~K}$ and $600 \mathrm{~K}$ is completely zipped and has become a normal CNT, while only 4 unzipped bonds rebounded at $400 \mathrm{~K}$. In Figure 3.3.b, the energy curve of CNT $(12,12)$ at $1000 \mathrm{~K}$ has higher values and shows the increasing energy during the selfhealing process, then becomes stable after the self-healing process is complete at $-2.14 \mathrm{eV}$. The same behavior occurs at $600 \mathrm{~K}$ but with lower energy. At $400 \mathrm{~K}$, the energy curve remains stable because there only 4 unzipped bonds rebounded during the simulation time. This is the relation between temperature, kinetic energy and total energy and it can be argued that temperature and kinetic energy result into a high energy. However, the behavior of the series based at the temperature $600 \mathrm{~K}$ confirms that the self-healing speed increases with the rising or increase in temperature as shown in Figure 3.3. 

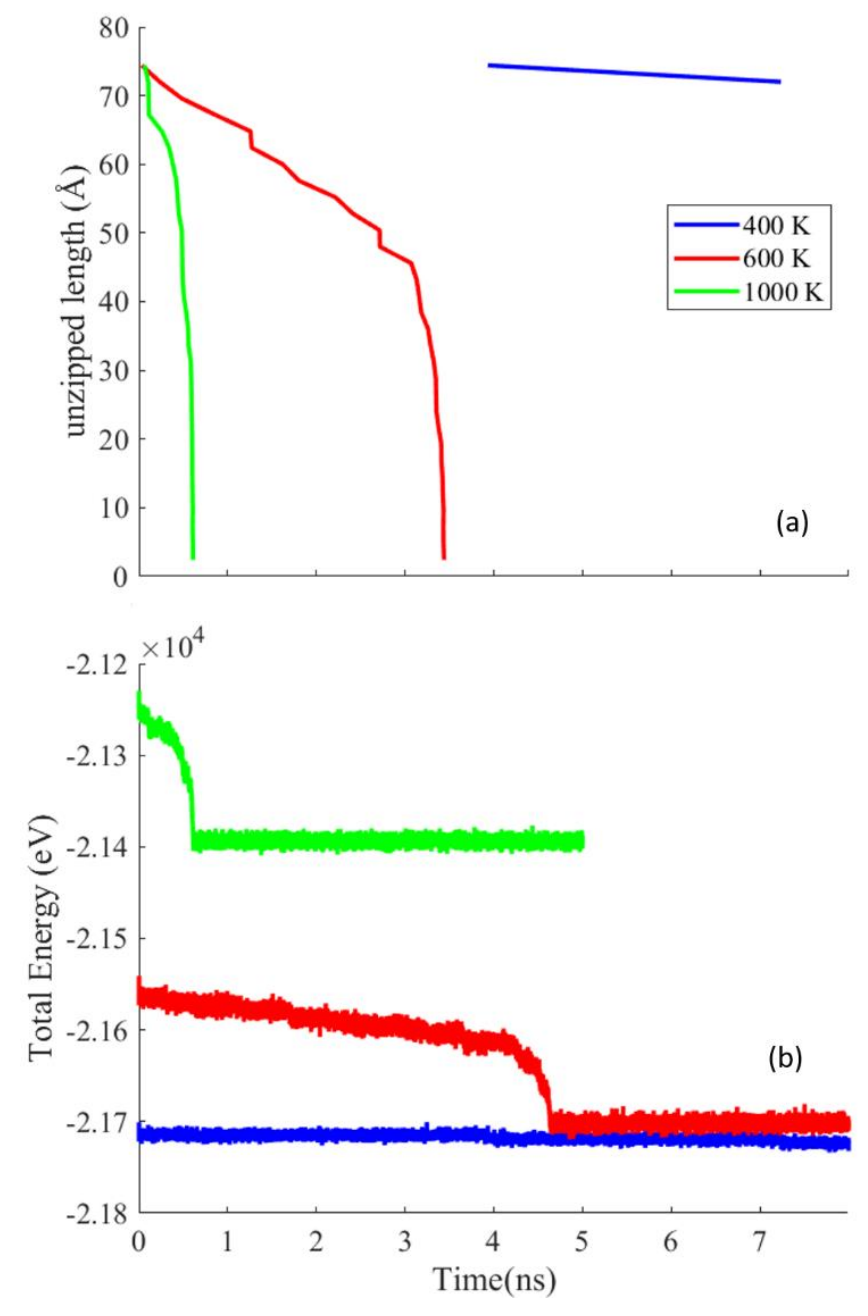

Figure 1.10 Partially unzipped $(12,12)$ CNT vs. simulation time with cutting length $L=70(\AA)$, relaxed at different temperatures $400 \mathrm{~K}, 600 \mathrm{~K}$, and $1000 \mathrm{~K}$. a) unzipped length b) total energy

\subsubsection{Structure}

Figure 3.4, represents three different unzipped structures $-1^{\text {st }}, 2^{\text {nd }}$, and the $3^{\text {rd }}$ at the same length of healing and total energy output vs. time of 3 unzipped CNT $(10,10)$ with a length of $400 \AA$ A configurations. They all have the same cutting length except the 1 st structure, which is unzipped until the end. From this structure, the unzipping length $L$ along in the model is $230 \AA$. It can also be seen that when the total energy decreases with time, the zipping length increases. 
This is due to the dangling bonds at the cut edge and this occurs at high temperatures and energy values ${ }^{108}$. This means that when the temperature of the system is high, the dangling edge loses its stability and results in a reconstruction behavior with unsaturated carbon atoms. This is why the 3rd structure zipped faster than the 2nd structure. The 3rd structure with two longitudinal nanoribbons shows two line of rebounded (L1, L2) and they zipped faster because all the atoms that needed to rebound were already in close proximity. In addition, multiple simultaneous rebounding minimizes the necessity of redoing edge reconstructions. The effects of the unzipped structure on self- healing, especially the speed of the zip in the three different structures: the 1st, 2nd, and 3rd, at temperature $1000 \mathrm{~K}$ depict that the self-healing property is strong and stable. The graphene nanoribbon (GNR) length also determines the bending levels of the CNT structures ${ }^{108}$. Therefore, since the GNR is dominant, the energy gains from the re-bonding process may lead to PUCNT structures rolling back to SWCNT structure and it may be energetically less favorable for small GNRs. This is because the unzipped GNR section has two primary folding directions, warp around longitude $\left(K_{l}\right)$ and transverse axis $\left(K_{t}\right)$ As shown by Tang et $a l^{108,111}$, in partially unzipped carbon nanotubes the GNR section resorts to a roll up at sufficiently with high temperatures, when $\left(K_{l}\right)$ override $\left(K_{t}\right)$. This process plays a major role in slowing the self-healing speed.

However, the three structures are different. The first structure is with one zip to the end, which makes that portion of the nano-tube a graphene sheet, while the 2 nd one has one zip, but not to the end, providing some gaps. As such, it can be concluded that the entire structures can self-heal and go back to normal due to reconnections of the carbon atoms as evident in the black curves: L1 and L2 shown in Figure 3.4. 

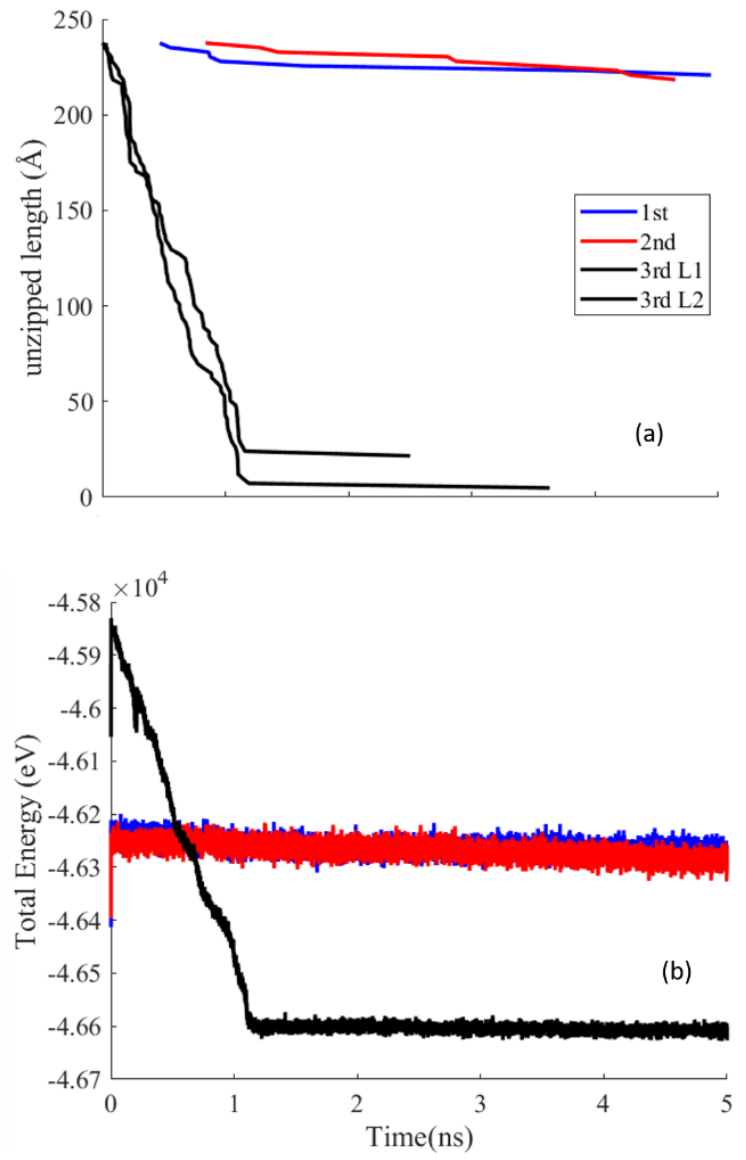

Figure 1.11 Structures of the first three partially unzipped $(10,10)$ CNT vs simulation time with cutting length relaxed at $1000 \mathrm{~K}$. a) unzipped length b) total energy

\subsubsection{Mechanical Properties}

\subsubsection{Uniaxial tension}

The uniaxial tension of the materials yields high strength and deep elongation at different relaxation testing. With the application of uniaxial tension in the Z-direction on all five CNTs $(10,10)$, the Unzipped Carbon Nanotubes curve depict high elongation and stable strength relaxation mode. Our studies were performed on the partially unzipped non-periodic systems at 300 K. For comparison we also performed the same calculation on a completely zipped CNT. 
Figure 3.5 shows our results for the six nanostructures, and depicts that the 3rd PUCNT structure and original CNT has the highest stress-strain value.

Furthermore, the Young modulus did not change between the unzipped CNT and the original tube, as shown in Table 3.1, which gives more value to the PUCNT in applications ${ }^{106-107}$.

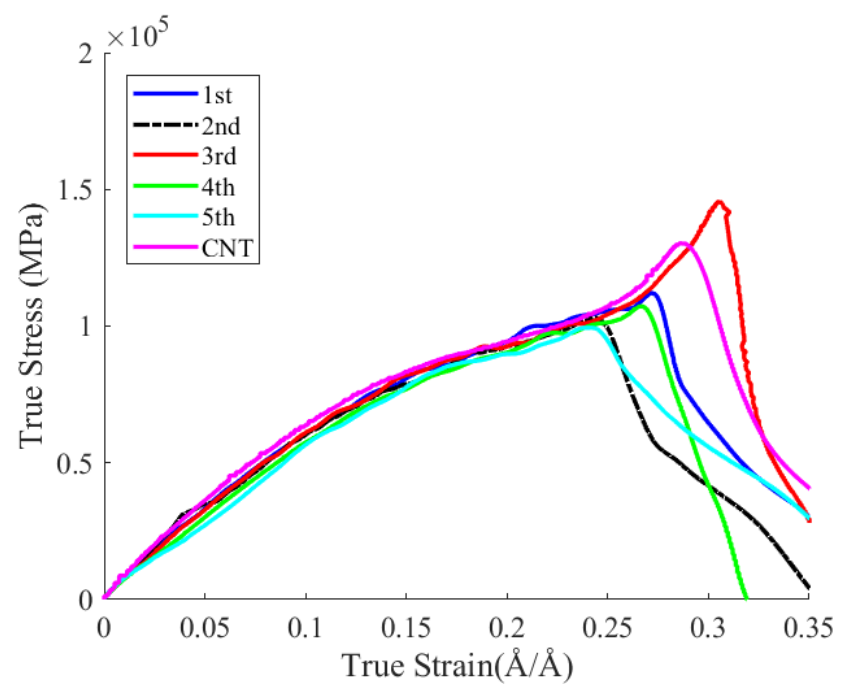

Figure 1.12 Stress-strain response for five partially unzipped carbon nanotube at $300 \mathrm{~K}$ 
Table 1.5 Overview of mechanical properties for different structures of unzipped CNT at $300 \mathrm{~K}$

\begin{tabular}{|l|l|l|}
\hline structure & \multicolumn{1}{|c|}{$(\mathrm{GPa})$} & Yield strength \\
& $(\mathrm{GPa})$ \\
\hline CNT & 0.60 & 129 \\
\hline $\mathbf{1}^{\text {st }}$ & 0.60 & 110 \\
\hline $\mathbf{2}^{\text {nd }}$ structure & 0.60 & 102 \\
\hline $\mathbf{3}^{\text {rd }}$ structure & 0.61 & 145 \\
\hline $\mathbf{4}^{\text {th }}$ structure & 0.57 & 107 \\
\hline $\mathbf{5}^{\text {th }}$ structure & 0.56 & 102 \\
\hline
\end{tabular}

\subsubsection{Temperature Effect:}

We studied the effect of different temperatures under uniaxial tension on the $1^{\text {st }}$ PUCNT structure, and our results are shown in Figure 3.6. We find that the temperature effects are quite small, as further shown in Table 3.2. In the temperature range of 300 to $900 \mathrm{~K}$, Young's modulus and the tensile strengths decrease with increasing temperature. Our findings of PUCNT agree with Dereli et al's ${ }^{116}$ results on CNT $(10,10)$ that it is brittle in low temperatures, between $300 \mathrm{~K}$ and $900 \mathrm{~K}$, and soft after $1200 \mathrm{~K}$ 


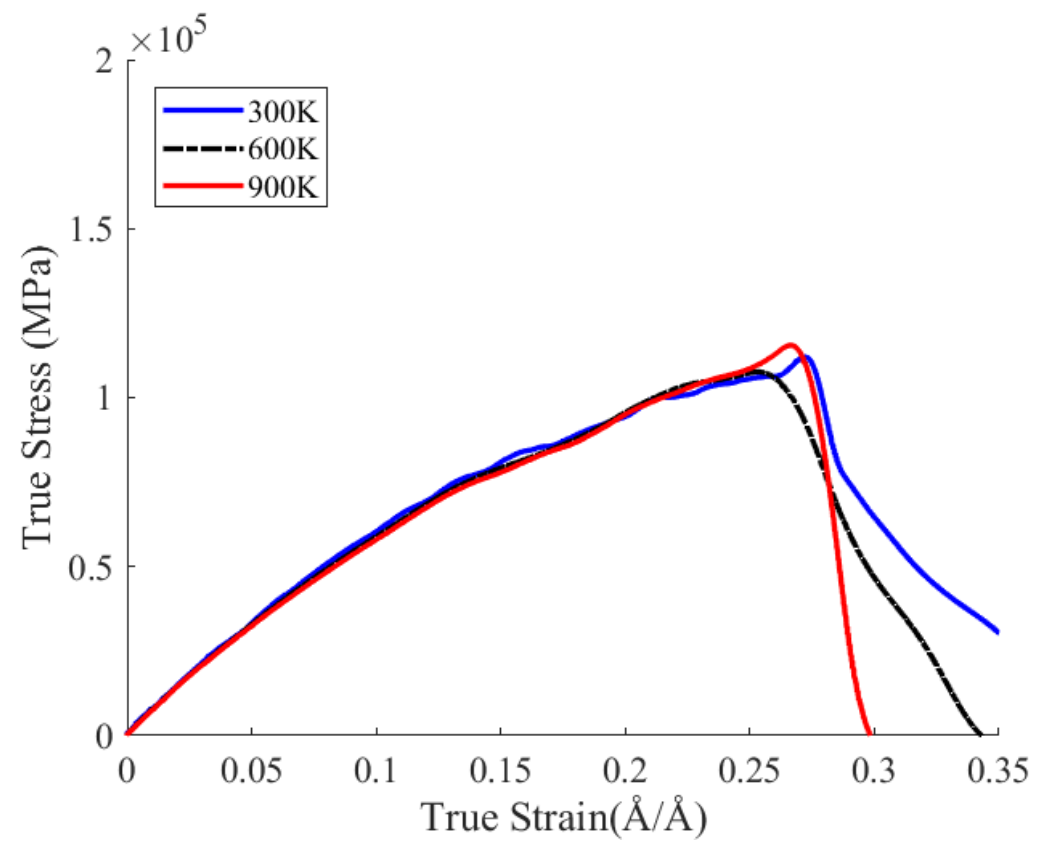

Figure 1.13 Temperature effect on stress-strain curve of 1 st structure.

Table 1.6 Mechanical properties for 1st structure under different temperature

\begin{tabular}{|l|l|l|}
\hline \multicolumn{1}{|c|}{ temperature } & \multicolumn{1}{|c|}{$\begin{array}{c}\text { Young modulus } \\
\text { (GPa) }\end{array}$} & \multicolumn{1}{c|}{$\begin{array}{c}\text { Yield strength } \\
\text { (GPa) }\end{array}$} \\
\hline $300 \mathrm{~K}$ & 0.60 & 110 \\
\hline $600 \mathrm{~K}$ & 0.58 & 107 \\
\hline $900 \mathrm{~K}$ & 0.57 & 115 \\
\hline
\end{tabular}

\subsubsection{Conclusion}

In conclusion, the analysis of the self-healing and the mechanical properties of Partially Unzipped Carbon Nanotubes (PUCNT) confirms that most of the structures tend to self-heal back to SWCNTs, with a different rate of healing at different temperatures. This is because they 
have a high reactivity level, especially due to their dangling bonds and stable local stress-strain at the junction. At different temperatures, the structure of the CNTs may bend at high levels in other directions. However, some PUCNTs may lose their self-healing properties where some edges are under tension from hydrogen atoms. Analysis of the CNT's mechanical properties also reveal that they have strong elongation and tensile strength. This means that they are a brittle structure at room temperature. CNTs and graphene in PUCNT structure also depicts a high elastic limit with different values of Young's modulus. This means that they can be applied in developing strong and stable electro-mechanical tools in different industries mainly due to their self-healing and mechanical properties. However, the temperature and length or size of the CNTs must be considered in developing these tools. Due to their mechanical and self-healing properties, the CNTs may be used in different devices such as radio frequency identification tags (RFID), health monitoring tools, switches, and wireless sensing devices. 


\section{CHAPTER IV}

\section{DAMAGE EVALUATION IN POLYETHYLENE COMPOSITE FROM MOLECULAR}

\section{DYNAMICS SIMULATION}

\subsection{Introduction}

Many MD simulations of polyethylene matrix with single-wall carbon nanotubes (SWCNT-PE) composite have been conducted to study a countless number of points including: interfacial mechanical properties ${ }^{117,118}$, improving the interfacial adhesion by chemical functionalization $^{119-124}$, glass transition temperature ${ }^{125,126}$, orientation and dispersion evolution of CNTs in the polymer matrix ${ }^{127}$, tensile strength with varying weight fraction ${ }^{128}$, and mechanical properties under static and dynamic tensile loadings ${ }^{129}$. Srivastava et al, ${ }^{130}$ reported in review papers about polymer/CNT composite that short nanotubes are a much harder, stiffer material compared to the polymer molecules by $40 \%$ and $30 \%$.Xiong et al. ${ }^{118}$ found that, the bond interaction plays a significant role in the load transfer of nanocomposites. They also found that the carbon nanotube embedded length and diameter has a great influence on the interfacial mechanical properties for nano reinforced composite. Frankland et al. ${ }^{131}$ performed extensive studies on the interfacial mechanical properties of reinforced nanocomposite using MD simulations and found that chemical functionalization of the nanotube surface can improve the interfacial adhesion. Tahreen et al. ${ }^{132}$ reported that the macroscopic rule-of-mixture is not an excellent approximation in the composite system because it assumes perfect bonding between the fiber and matrix. Liu et al. ${ }^{133}$ showed that the mechanical properties of composites are dependent 
on temperature and loading rates. Also, the Young's modulus and interaction energy reach the largest values when the CNTs have the highest aspect ratios. All MD simulations share a commonality that nanocomposite toughness increases with enhanced interfacial adhesion. Although polymer improved specific strength and modulus by reinforcing the SWCNT, the embrittlement gained from filler is a significant challenge common to polymer composites with micron fillers and nanofillers ${ }^{124-126}$. To prevent crack initiation and propagation in brittle polymers and their composites, different strategies were developed ${ }^{122,127-131}$. The role of void nucleation, growth, and coalescence during plasticity in polymer/ CNT composites is rarely investigated. Goh et al. ${ }^{134,135}$, showed that the number of cycles to failure under fatigue decreases with increasing weight fractions of carbon nanotubes in the composite. Arora et al. ${ }^{136}$ experimentally investigated the elasto-plastic deformation by stress-strain curves behavior with void effect of high-density polyethylene composite with CNT enforced randomly. They found that the maximum stress of the model with $1 \%$ voids is lower than the maximum stress of the model without the voids by $9 \%$. Dasari et al. ${ }^{137}$, report that the plastic damage occurring in a polymer composite is caused by formation of numerous voids in a polymer matrix. Molecular Dynamics (MD) simulations provide a useful tool for understanding the mechanisms of damage at lower length scales. To study the nanoscale mechanisms of void nucleation, growth, and coalescence during plasticity damage, Huddleston $e a^{88}$ presented a significant atomistic simulations mythology for studying damage systems at the nanoscale level by calculating the void number density (void nucleation) and void volume during the deformation of the system. Bowman $e$ t $a l^{138,139}$ provided an extensive studying about structure evolution of polyethylene during a plastic damage. They examined the smallest configurations of PE in periodic system that can represent the mechanical properties and damage behavior of bulk amorphous 
polyethylene. Their results shown that a polyethylene with 16 chains and 267 monomers length shows the smallest configuration that the mechanical and damage properties are converged.

In this work, evaluation damage response of a mechanism of polyethylene nanotube composite was studied by following Bowman et al's ${ }^{138,139}$ work in pure polyethylene to explore the variance of the results between polymer composites and polymer. Under stress-strain responses at different temperatures, strain rates, and stress states the corresponding damage evolution of the SWCNT-PE is presented in this chapter. The Modified Embedded Atom Method with Bond Order (MEAM-BO) force formalism for unsaturated hydrocarbons is utilized in this chapter to perform a study for the nanoscale mechanisms of void nucleation, growth, and coalescence during plasticity damage in a SWCNT-PE composite. MEAM-BO is modified from the MEAM $^{79}$ potential so MEAM-BO has excellent representation of free surfaces and capacities of bond forming and breakage similar to MEAM. The term of background electron density in the MEAM-BO equation allows the capture of the local state of atoms and provides a high accuracy description of the free volume evolution during deformation. We proposed to use MEAM-BO to study the plasticity and damage response of polymer composites. However, because MEAM-BO is not ready in LAAMPS packaging at this time we used MEAM for the polyethylene since it works for saturated hydrocarbons, REBO for the SWCNT, and a Lennard-Jones (LJ) interatomic potential for the nonbonding interaction between SWCNT and PE. The purpose of the present work is to provide a understanding of damage mechanics at the molecular level, which is critically important as damage is a microscopic phenomenon. 


\subsection{Method}

\subsubsection{Model generation of SWCNT composite and Simulation Setup}

A $(10,10)$ SWCNT with length $35 \AA$ reinforced in a polyethylene matrix with 16 chains and a chain length of 267 monomer units was constructed for use in this calculation with total number atoms 27064. Polyethylene structures were determined according to Bowman et al. 's 138 results. They show that the system size of polyethylene to capture the damage behavior should be large enough to have the mechanical properties converging in periodic systems. Also they conclude that the converting caused with more than 16 chains and 200 monomers with only a slight dependence on molecular weight. Geometry optimization of the structure is performed by the force field Dreiding potential. After the initial construction and optimization process, the model was minimized within LAMMPS ${ }^{94}$ using a combination of potentials in a hybrid style. The REBO ${ }^{80}$ Potential was used for carbon-carbon interactions in the nanotube. The Modified Embedded-Atom Method (MEAM) ${ }^{79}$ was used to define the interactions between $\mathrm{H}$ and $\mathrm{C}$ atoms in the polyethylene (PE), and the Lennard-Jones (6-12)(LJ) interatomic potential represented the nonbonded interfacial interaction between the CNT and the polyethylene. The vdW parameters in the $\mathrm{LJ}$ potential were set as, $\varepsilon=0.4492 \mathrm{~kJ} / \mathrm{mol}$ and $\sigma=0.401 \mathrm{~nm}^{140}$. The equilibration protocols were performed with a 0.4 fs time step using an isothermal-isobaric Nose-Hoover ensemble (NPT) at $250 \mathrm{~K}$ and zero pressure for about 1000ps until the temperature, pressure, density, and total energy of the system fluctuated around a constant value thereby ensuring it was well equilibrated before we continued for $10 \mathrm{~ns}$. For the temperature dependence study, the system equilibrated by NPT at $150 \mathrm{~K}$ and $350 \mathrm{~K}$ for an additional $1 \mathrm{~ns}$ to relax the system at the new temperature. Once equilibrated, the simulation cell (70 $\mathrm{A} \times 70 \AA \times 86 \AA)$ was forced to various uniaxial and biaxial stress states (tension, shear, compression) and constant stress levels with 
NPT dynamics until complete fracture with the corresponding strain rate $3 \times 10^{9} s^{-1}$. For the strain-rate sensitivity measurement, three different strain rate values are utilized to investigate the role of strain rate on several mechanical parameters.

\subsubsection{Damage identification processing method}

A similar method was used in Huddleston et $a l^{88}$ and Bowman et al ${ }^{138}$ to calculate the accumulating damage in copper and polyethylene systems respectively. Atomic structure snapshots were taken at various time intervals during the simulation in order to investigate the damage evolution and structural changes that occur during deformation. The atomic visualization software OVITO ${ }^{141}$ was used to construct a surface mesh by using a Delaunay triangulation of the atomic coordinates in the system. This enabled further study of the damage incurred. Then, the solid and free volume of the polymer was calculated by using a spherical probe with a radius of $3.4 \AA$ to check the tetrahedra for solid and empty regions. The probe radius of $3.4 \AA$ is slightly larger than the interchain distance between adjacent polyethylene chains and the distance between PE and SWCNT. Lastly, a custom python script created to count the number of empty regions (voids), to determine the volume of each individual void, and to calculate the geometrical centres of each void.

\subsection{Results and Discussions}

\subsubsection{Uniaxial Tension}

The Composite of SWCNT with PE was pulled vertically along the longitudinal CNT direction ( y-direction ) at $250 \mathrm{~K}$ with a strain rate of $3 \times 10^{9}$. In Figure 4.1, the stress-strain curve response shows four sections: a) elastic, b) yielding, c) strain softening, and d) strain hardening regions. Stress increases linearly in the elastic region with a Young modulus of 1.46 
GPa which means the Polyethylene composite with SWCNT has higher stiffeness then pure PE $(0.92 \pm 0.1 \mathrm{GPa})^{139}$. The Young's modulus was calculated as the slope of the elastic region where the strain is less than 0.05 . The stress increases monotonically until reaching a yield stress of about 94.93 MPa while the stress at yield reaches only $42.45 \pm 2.44 \mathrm{MPa}$ in PE. A $0.5 \%$ strain offset is used to determine the yield strength. After yielding, an unsteady behavior can be observed followed by the strain softening regime.

Finally, the response of stress-strain curve changes, with stress increasing nonlinearly until failure occurs either by chain scission or by chains pulling away from entanglement clusters. Bowman et al, ${ }^{139}$ reported that the stress-strain curve response of a stretched pure polyethylene can be divided into 4 sections: an elastic region, a yielding section, a stress plateau, and a strain hardening section. In the current results of the polyethene composite with SWCNT, the stress-strain behavior does not show a definitive plateau region. Instead, an unsteady region is observed followed by a softening region. This is due to the heterogeneity of the interface between the polyethylene and $\mathrm{SWCNT}^{142}$. 


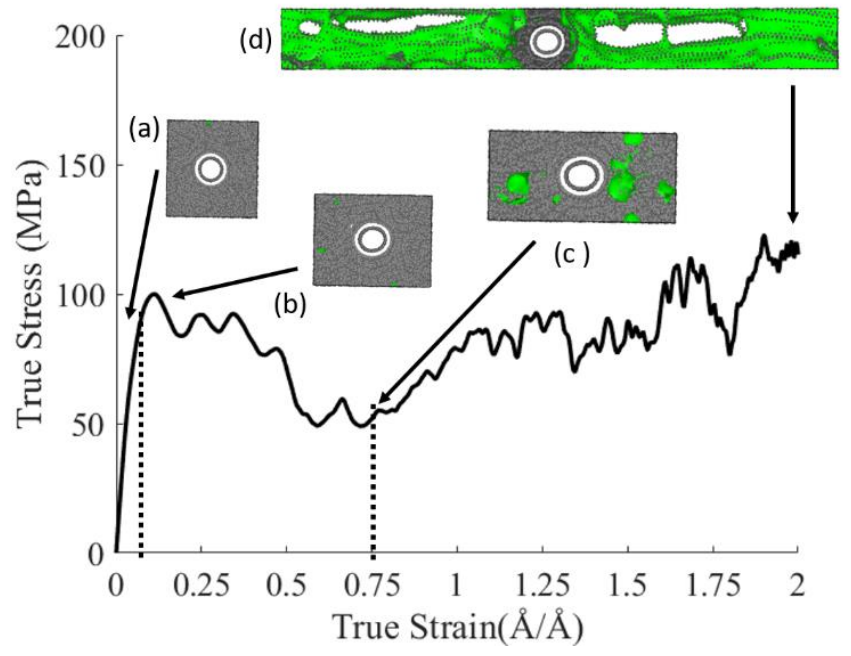

Figure 1.14 Stress-Strain curve response of stretched polyethylene composite with single wall carbon nanotube (SWCNT-PE) in y-direction

The response of damage evolution of the composite is illustrated in Figure 4.2 which shows the void number density, the average void size, and the total damage (void volume fraction) together with the stress-strain curve. Figure $4.2 \mathrm{~b}$ presents the number density of voids in the composite as a function of strain. The void number density includes both the nucleation and coalescence phenomenon due to difficulty in separating the coalescence from the void number density. Nucleation events increase the void number density while coalescence decreases the void number density by merging of void surfaces. Nucleation and coalescence can occur simultaneously, resulting in a complex behavior of the evolution of the void number density. The void number density evolution can be divided into four flex points as shown in Figure 4.2b. Up to a strain of 0.13 , the number of voids rapidly increases as the material approaches yielding. Once yielding occurs (first blue vertical line in Figure 1.2) nucleation proceeds but at an ever decreasing rate until the maximum nucleation threshold is achieved (strain of 0.31 ) and the nucleation rate becomes negative due to void coalescence as the coalescence of voids is more 
dominate than nucleation of voids. Thus, the softening of the stress-strain response is attributed to the rapid coalescence of voids. The next flex point is observed at a strain of 0.75 , where the decrease in the void number occurs at a much slower rate until reaching a strain of 1.31 (last flex point) after which the void number density is nearly constant as failure begins to occur in a localized region.

Figure 4.2c shows the average void volume evolution. From this region, three flex points can be observed (red dotted lines). Initially, the void volume associated with the inner nanotube interface region produces a significant initial void volume. In region 1, the average void volume decreases due to a nucleation dominated regime where small voids nucleate driving down the average void volume fraction. In this regime coalescence events are not frequent as initial voids are small and separated from each other. This is in agreement with the void number density curve (Figure 4.2b) where rapid increase in the void number density can be observed. The second region occurs at a strain level of $0.31-0.75$. This region is characterized by a rapid increase in the average void volume due to the growth and coalescence of existing voids. In the remaining two regions the average void volume continues to increase, but at a slower rate. Figure $4.2 \mathrm{~d}$ represent the evolution of the total damage (total free volume fraction) as a function of strain. The damage increases with strain. However, at a strain of 0.4 an accelerated increase in the damage can be observed. This is due to the growth and coalescence of voids, resulting in larger total free volume 


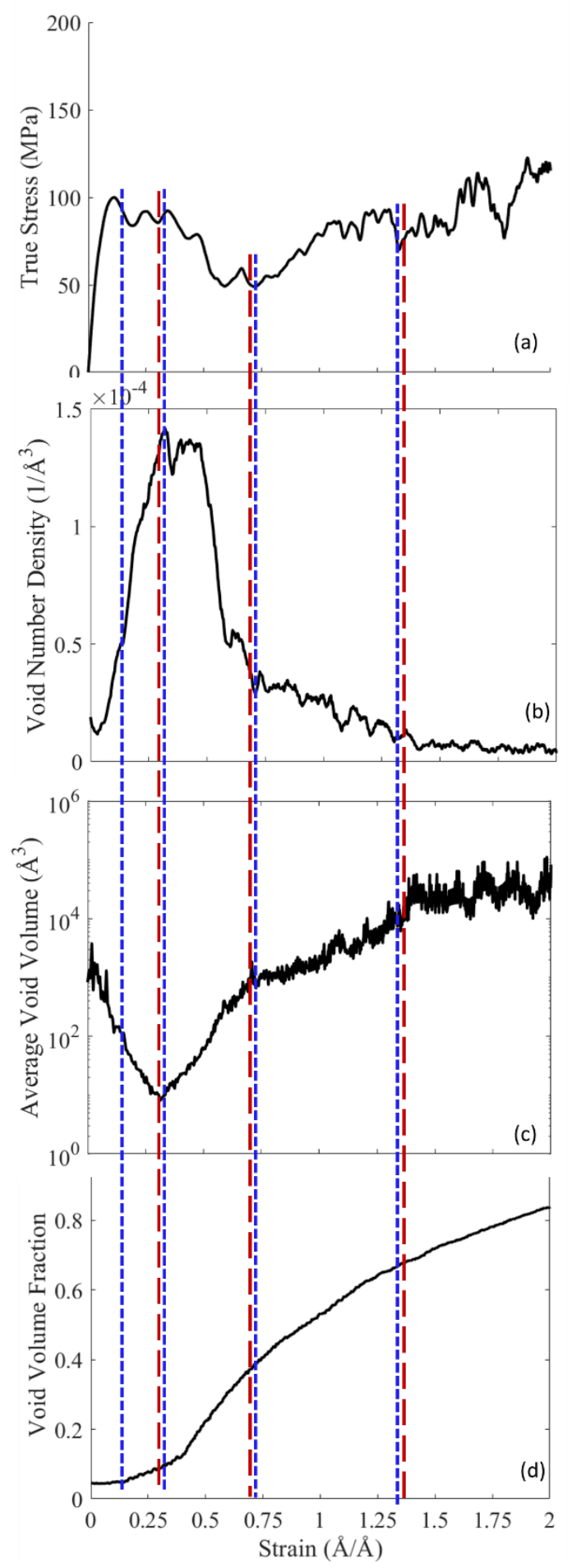

Figure 1.15 Damage evolution during uniaxial tensile deformation at $250 \mathrm{~K}$ illustrating (a) the stress-strain response, (b) void number density, (c) average void size, and (d) the overall damage (void volume fraction). 


\subsubsection{Strain Rate Dependence}

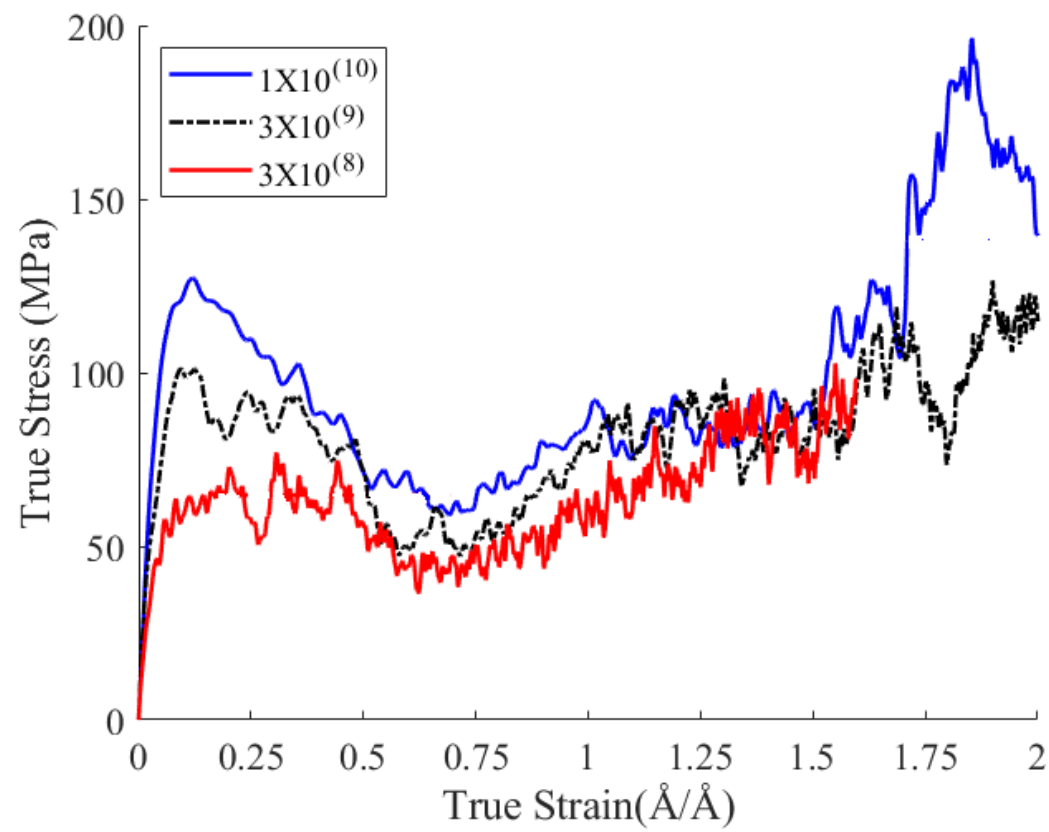

Figure 1.16 Stress-strain behavior of polyethylene composite beads SWCNT deformed at different strain rates at $250 \mathrm{~K}$

Figure 4.3 shows the strain rate dependent behavior of a SWCNT-PE composite deformed at $250 \mathrm{~K}$ under uniaxial tensile load in the y direction with different strain rates. The yield strength depends on the strain rate, as observed from the figure. However, after yielding, the stress-strain curve shows similar behavior for all strain rates. This indicates that the damage response of the material is not dependent on the strain rate. Details of the damage response is shown in figure 4.4. The maximum void number density is dependent on the strain rate which corresponds to the yield point dependence (Figure 4.4a). After the maximum void number density is reached, the evolution of the void number density, average void volume, and void volume fraction all show the same behavior, indicating that damage behavior is not dependent on the strain rate. 

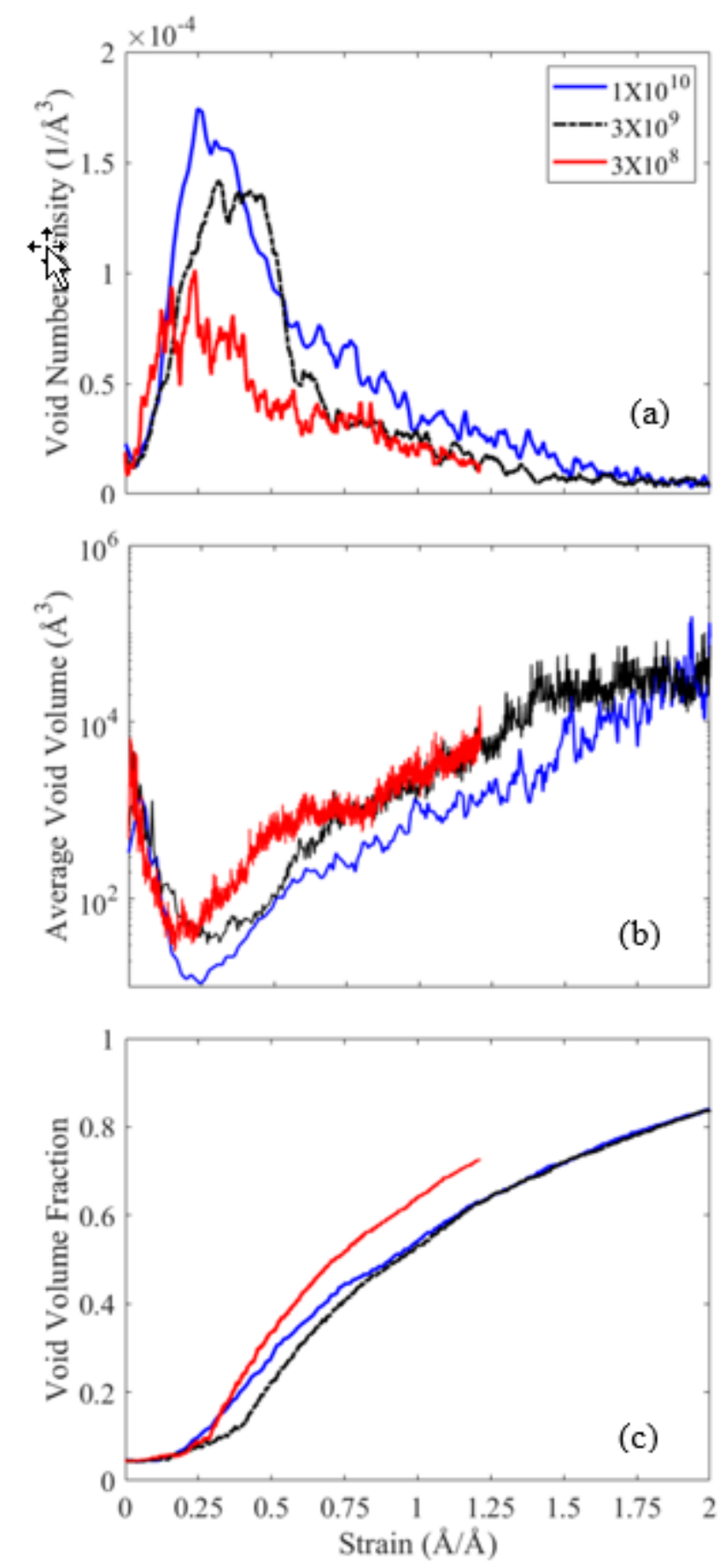

Figure 1.17 Damage evolution during uniaxial tensile deformation at various strain rate at a temperature of $250 \mathrm{~K}$ illustrating (a) void number density, (b) average void size, and (c) the overall damage (void volume fraction). 


\subsubsection{Stress state Dependence}

To analyze the effect of a stress state on the mechanical behavior of the composite SWCNT-PE, different loading test conditions were applied at a von Mises equivalent strain rate of $3 \times 10^{9}$. Figure 4.5 exhibits the equivalent stress-equivalent strain behavior for the different stress states. From the figure, it can be observed that the modulus of elasticity is higher for loading in compression and biaxial tension than for shear and tension. Also, the yield stress is higher for compression and biaxial loading. However, for a biaxial tensile loading condition, a sharp strain softening regime occurs immediately after yield. The observed trends for the mechanical behavior of composite SWCNT-PE as a function of stress state is comparable with Bowman's result for polyethylene ${ }^{139}$. However, the yield strength for composite SWCNT-PE is higher than polyethylene as shown in Table 4.1.

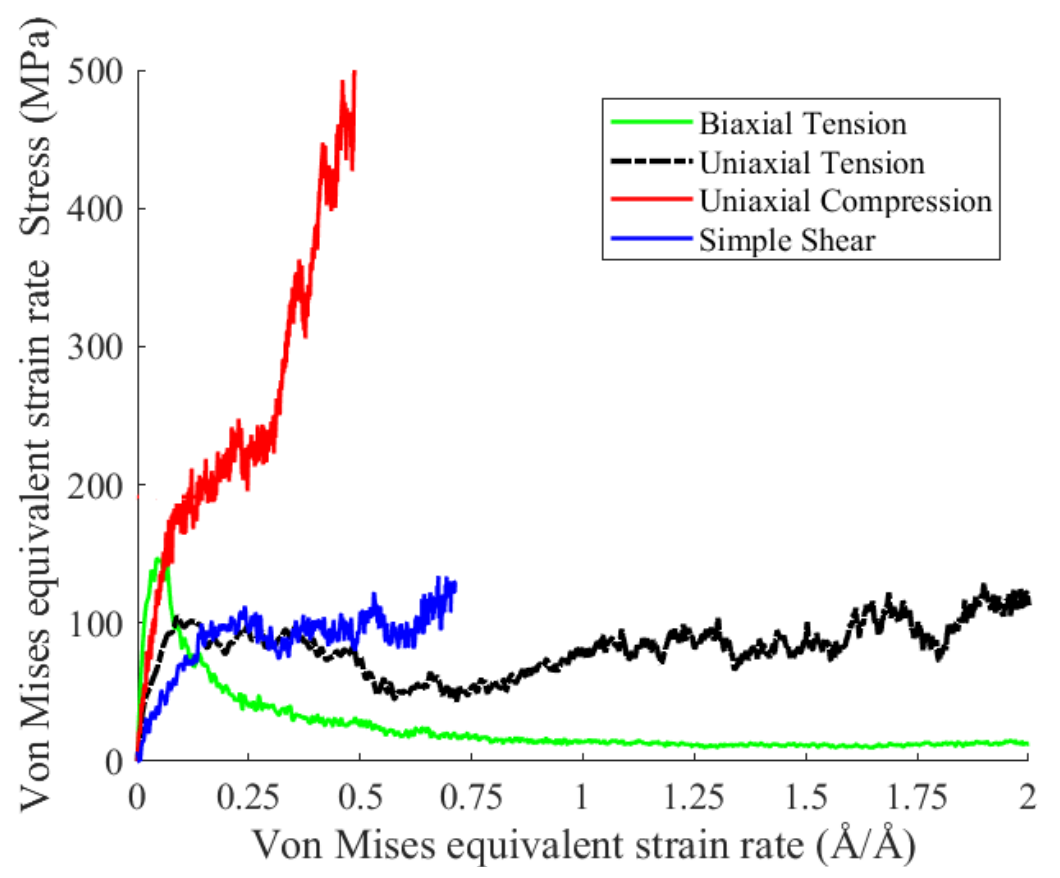

Figure 1.18 Equivalent stress-strain Curve at a temperature of $250 \mathrm{~K}$ and a strain rate of $3 \times 10^{9} / \mathrm{s}$. under different stress states 
Table 4.1 shows the overview of the mechanical properties for different stress states, temperatures, and strain rates for the composite SWCNT-PE. Bowman's results for PE is included in the table for comparison.

The damage behavior as a function of strain is illustrated in Figure 4.6. The number of voids increases sharply for biaxial and gradually for uniaxial tension until a maximum point is reached at a strain of 0.08 and 0.3 , respectively (Figure 4.6a). After reaching the maximum void number density, a rapid decrease is observed for both the biaxial and uniaxial tensile loading conditions due to void coalescence. Also, it can be observed that the rate of void nucleation is highest for the biaxial tension due to the stress triaxiality effect.

The number density of voids for simple shear increases gradually. However, no definitive decrease in the number density of voids is observed until failure. The void number density is lowest for the compression case due to the immediate collapse of voids after nucleation.

Figure $4.6 \mathrm{~b}$ shows the average void volume verses strain for different stress triaxialities. For the biaxial and uniaxial tension, the average void volume decreases until reaching a minimum at a strain of 0.08 and 0.3 , respectively. This is due to the nucleation of voids at this initial stage. Thereafter the average void volume increases due to growth and coalesce for both the biaxial and uniaxial tension, until failure. It is also observed that the average void volume is higher for the biaxial tension case due to the stress triaxiality effect. The evolution of average void volume is similar for both simple shear and compression tests. For both cases, the average void volume decreases until failure due to the collapse of voids. The overall damage in figure $4-5 \mathrm{c}$ shows that for shear and compression, the failure of the system occurs at lower stain levels compared to the biaxial and uniaxial stress cases. Table 4.1 shows the overview of the mechanical properties for 
different stress states, temperatures, and strain rates for the composite SWCNT-PE. Bowman's results for PE is included in the table for comparison.

The damage behavior as a function of strain is illustrated in Figure 4.6. The number of voids increases sharply for biaxial and gradually for uniaxial tension until a maximum point is reached at a strain of 0.08 and 0.3 , respectively (Figure 4.6a). After reaching the maximum void number density, a rapid decrease is observed for both the biaxial and uniaxial tensile loading conditions due to void coalescence. Also, it can be observed that the rate of void nucleation is highest for the biaxial tension due to the stress triaxiality effect.

The number of voids in Figure 4.6a for simple shear increases gradually. However, no definitive decrease in the number density of void is observed until failure. In the compression case, the void number density is lowest due to the immediate collapse of voids after nucleation. Figure $4.6 \mathrm{~b}$ shows the average void volume verses strain for different stress triaxialities. For the biaxial and uniaxial tension, the average void volume decreases until reaching a minimum at strain of 0.08 and 0.3 , respectively. This is due to the nucleation of void at this initial stage. After which the average void volume increases due to growth and coalesce for both the biaxial and uniaxial tension until failure. It is also observed that the average void volume is higher for the biaxial tension case due to the stress triaxiality effect. The evolution of average void volume is similar for both simple shear and compression test. For both cases, the average void volume decreases until failure due to the collapse of void. The overall damage in figure $4-5 \mathrm{c}$ shows that for shear and compression, the failure of the system occurs at lower stain levels compared to the biaxial and uniaxial stress cases. 

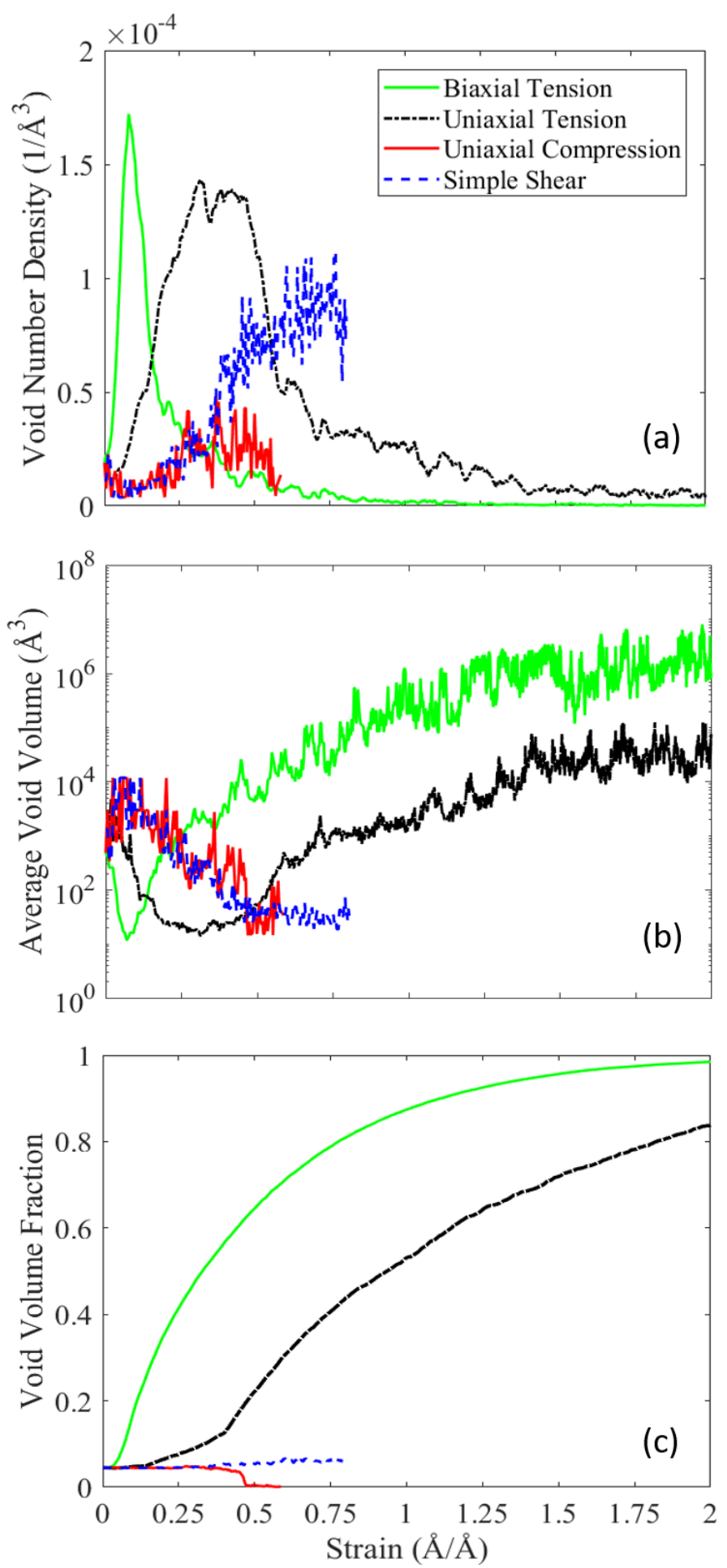

Figure 1.19 Damage evolution during different stress state with strain rate $3 \times 10^{9}$ showing (a) void number density, (b) average void size, and (c) the void volume fraction 


\subsubsection{Temperature Dependence}

Understanding the effect of temperature on the mechanical behavior of SWCNT-PE composite is important for its application in thermal interface materials.

In Figure 4.7, the stress- strain response of a SWCNT-PE composite at three different temperatures $150 \mathrm{~K}, 250 \mathrm{~K}$ and $350 \mathrm{~K}$ shows the temperature-sensitivity of the young modulus, elastic boundary, and the shape of the hardening curve. The nanocomposite has a glass transition (Tg) temperature of $\sim 320 \mathrm{~K}^{115}$ while PE has $255 \mathrm{~K}^{139}$. The stress-strain behavior changes as the polymer transitions from a glassy state at $150 \mathrm{~K}$ and $250 \mathrm{~K}$ to a rubbery state at $350 \mathrm{~K}$. It is observed from Figure 4.7 that the uniaxial tensile yield point decreases as the temperature transitions from the glassy to the rubbery state (150K-350K). Also, a strain softening section follows yield for temperatures at $150 \mathrm{~K}$ and $250 \mathrm{~K}$. Conversely, at $350 \mathrm{~K}$ a steady stress behavior is observed with no strain softening after the yield point.

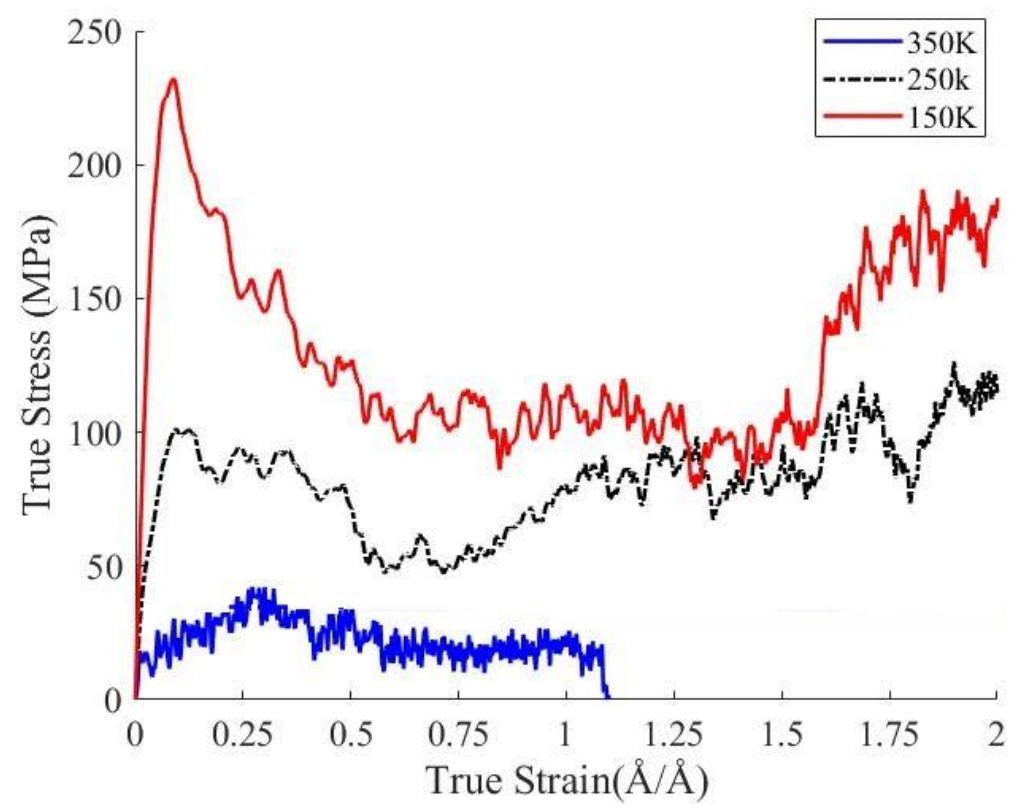

Figure 1.20 Dependent stress-strain response of SWCNT-PE at strain rate $3 \mathrm{X} 10^{9}$ 
The evolution of damage illustrated by the void number density, average void size, and void volume fraction are shown in figure 4.8. The temperature dependence of the damage response is clear from these figures. In Figure 4.8a, the number of voids increases for all temperatures until a maximum value is reached at the yield point, then the void number density rapidly decreases due to the simultaneous nucleation and coalescence growth. The maximum number density of voids is influenced by the thermal expansion, especially in the rubbery regime. Also, the average void volume exhibits the same behavior for all temperatures. During the nucleation phase, the average volume of voids decreases due to the formation of small voids, and after nucleation ceases the growth and coalescence of voids leads to a steady increase in the average void volume (Figure $4.8 \mathrm{~b}$ ). The average void volume decreased with an increase in temperature. The same results were shown by Bowman's study ${ }^{139}$ of pure polyethylene, except that the $250 \mathrm{~K}$ curve is closer to the $150 \mathrm{~K}$ curve in this study because they are in the same state (glassy). While in Bowman's study, the $250 \mathrm{~K}$ curve is closer to the glass transition (Tg) temperature of PE, which is $255 \mathrm{~K}$. 
Table 1.7 Overview of mechanical properties for SWCNT-PE under different conditions

\begin{tabular}{llclc}
\hline simulation & \multicolumn{2}{c}{ Elastic Modulus (GPA) } & \multicolumn{2}{c}{ Yield Stress (MPA) } \\
& SWCNT-PE & PE (139) & SWCNT-PE & PE (139) \\
\hline Strain Rate (uniaxial Tension) & & & & \\
1x10 & 1.79 & $1.85 \pm 0.18$ & 122.4 & $87.2 \pm 3.97$ \\
$\mathbf{3 x 1 0}^{\mathbf{9}}$ & 1.46 & $0.92 \pm 0.1$ & 91.87 & $42.45 \pm 2.44$ \\
$\mathbf{3 x 1 0}$ & 0.89 & $0.44 \pm 0.22$ & 63.81 & $11.1 \pm 1.1$ \\
Stress State 250k, 3X10 $^{9}$ ) & & & & \\
Uniaxial Tension & 1.46 & $0.92 \pm 0.1$ & 91.87 & $42.45 \pm 2.44$ \\
compression & 2.12 & $1.7 \pm 0.23$ & 182.7 & $57 \pm 9.8$ \\
shear & 1.29 & $1.47 \pm 0.18$ & 98.32 & $45.2 \pm 6.0$ \\
Temperature (uniaxial Tension) & & & & \\
350K & 0.17 & $0.24 \pm 0.03$ & 43.46 & $10.3 \pm 0.29$ \\
$\mathbf{2 5 0 K}$ & 1.46 & $0.92 \pm 0.1$ & 91.87 & $42.45 \pm 2.44$ \\
$\mathbf{1 5 0 K}$ & 3.79 & $3.62 \pm 0.21$ & 224.1 & $145.7 \pm 6.15$ \\
\hline
\end{tabular}



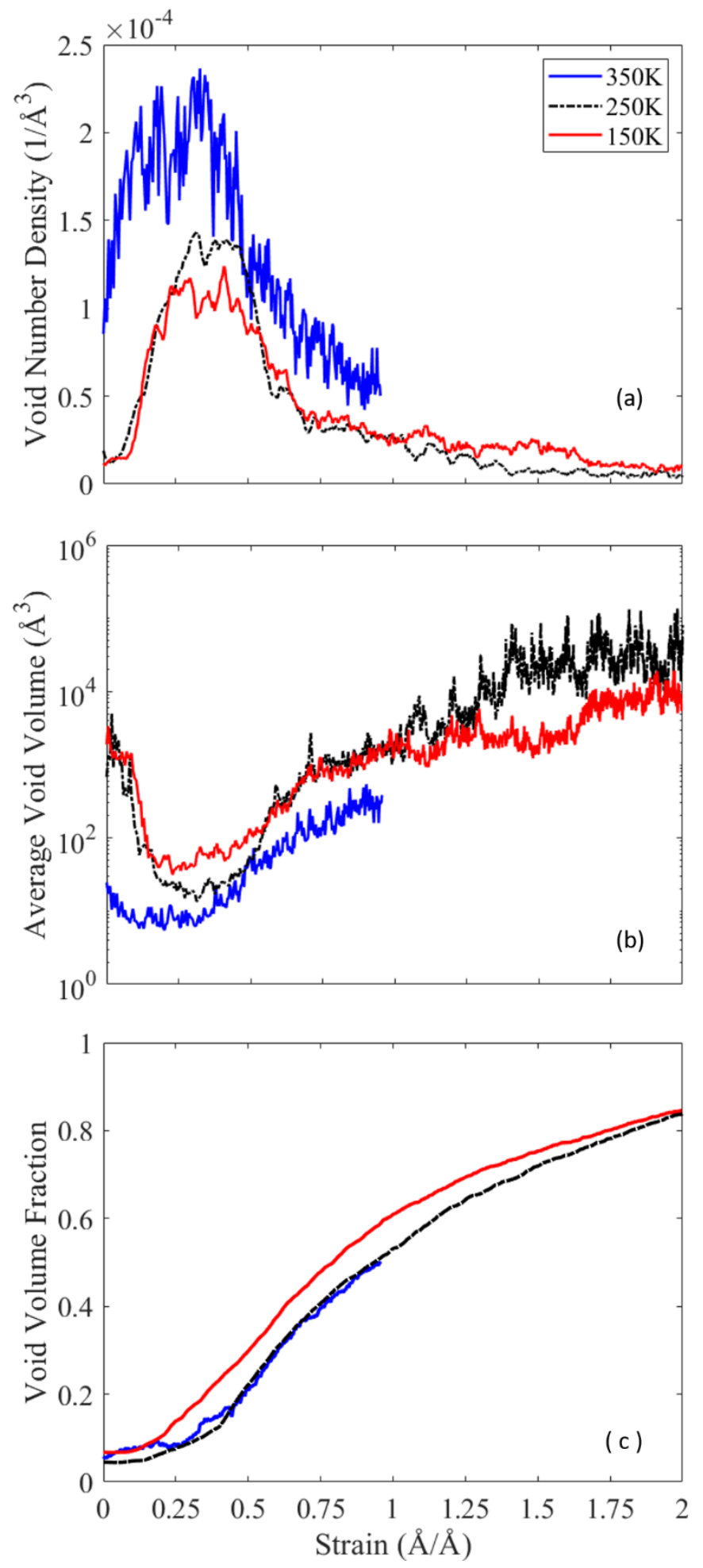

Figure 1.21 Damage evolution during uniaxial tensile with strain rate $3 \mathrm{X} 10^{9}$ at different temperatures showing (a) void number density, (b) average void size, and (c) the void volume fraction. 


\subsubsection{Conclusions}

In In this chapter, stress-strain response and the mechanisms of damage at lower length scales of a SWCNT-PE composite was investigated under various stress states, strain rates, and temperatures by using molecular dynamic simulations. Damage evolution analysis was performed by analyzing the void number density, mean void size, and void volume fraction during the deformation.

The yield strength and Young's modulus show high strain rate sensitivity. However, the flow stress was not sensitive to the strain rate. Also, a strain rate independent damage behavior was observed after yielding, so the stress-strain response shows a similar behavior for all strain rates.

The stress state sensitivity of damage distribution in a SWCNT-PE composite was analyzed under uniaxial tension, biaxial tension, compression, and shear at $250 \mathrm{~K}$ and a strain rate of $3 \times 10^{9}$. It was observed that the modulus of elasticity is higher for loading in compression and biaxial tension than for shear and tension. The most damage was observed under biaxial and uniaxial tension, with a higher rate of nucleation and growth of voids in biaxial compared with uniaxial tension. The overall damage for shear and compression shows the failure of the system occurs at lower stain levels compared to the biaxial and uniaxial stress cases.

Finally, the temperature dependence of the Young's modulus, elastic boundary and the shape of the hardening curve was studied. At the glassy state $(150 \mathrm{~K}$ and $250 \mathrm{~K})$, a strain softening regime is observed after yield, followed by a strain hardening regime. For the rubbery state $(350 \mathrm{~K})$ a steady stress behavior is observed, instead of the strain softening between the yield point and the strain hardening. The evolution of damage curves shows that at lower temperatures, 
damage is caused by the nucleation and growth of voids, but at higher temperatures the failure is caused by the coalescence and grown of voids.

In overview, comparing the stress-strain curve and damage response in pure polyethylene and a polyethene based SWCNT composite, we found that SWCNT improves the mechanical and the thermal properties of the material. The damage evolution of the composite is governed by nucleation of voids, until a maximum number density of voids is reached at the yield point followed by the growth coalescence stage until failure. In PE the nucleation and coalescence happens simultaneous until a stress plateau region is reached prior to the harden stage.

Also, the big difference in the mechanical behavior observed between the PE and PE composite is related to the temperate sensitivity due to the difference in the $\mathrm{Tg}$ values between the two materials. 


\section{CHAPTER V \\ SUMMARY AND FUTURE WORK}

\subsection{Summary}

In this work, we report the results of molecular dynamic and energy minimization studies of carbon-based nanostructures. We demonstrated the capability of the Modified Embedded Atom Method with Bond Order (MEAM-BO) potential to capture single-wall carbon nanotube (SWCNT) properties accurately. We illustrated this by calculating the wavenumber of the radial breathing mode $(\mathrm{RBM})$, elastic properties, and folding energy $(\Delta \mathrm{E})$, which are all associated with bond curvature. We found that the MEAM-BO potential results correlate with experimental and Density Functional Theory (DFT) results except for the folding energy results. Consequently, we updated the MEAMBO potential parameters to produce a MEAM-BO* potential for the SWCNT system. Interestingly, including the SWCNT data base improved the previous fit of the $\mathrm{C}-\mathrm{H}$ system. Previous studies in the literature concluded that the nanotube diameter, $d$, inversely affected the mechanical properties of SWCNTs, and MEAM-BO showed that the wavenumber of the RBM and the folding energy are both inversely proportional to the SWCNT diameter. Also, the MEAM-BO SWCNT's Young's Modulus followed a quadratic behavior with respect to the SWCNT diameter. When comparing MEAM-BO*, REBO, and ReaxFF with DFT results, we found that MEAM-BO* gave the closest results to DFT for the RBM wavenumbers, folding energy, and SWCNT Young's modulus, especially for small diameter SWCNTs. We conclude that MEAM-BO* captures SWCNT curvature effects and unsaturated hydrocarbon bond behavior. Thereafter, five different partially unzipped carbon 
nanotubes (PUCNT) were investigated for their mechanical properties and the self-heling of the simplest PUCNT by using molecular dynamics simulations.

In addition, we studied a polyethylene single wall carbon nanotube (SWCNT-PE) composite's mechanical properties and damage evolution. In order to capture the varying effects of strain rate, temperature, and stress state for the damage of the composite, damage was computed in terms of the void number density (void nucleation) and void volume and we compared the results with the previous studies ${ }^{138,139}$ about polyethylene to catch the load transfer between the matrix and CNT. The ISV model for SWCNT-PE can be the next step to upscaling the relationship between void nucleation and damage observed in molecular dynamics to higher length scales by using a finite element analysis (FEA).

\subsection{Future Work}

Molecular dynamic simulations are continuing progress in development and uses in various fields. Force fields are the heart of MD simulations, and working on developing their performance is a significant area. In Chapter II, we modified some of the MEAM-BO parameters and produced MEAM-BO* to get accurate calculations, especially for folding energy results. For future work, MEAM-BO* needs be used for SWCNT composites and further tested.

Also, predicting the bond length and the bond angles of the molecules with unsaturated bonds in MEAM-BO* is reasonable for studying interfacial damage of complex biomaterials in terms of void nucleation, growth, and coalescence under the stress-strain responses and the corresponding damage evolution with different strain rates. ISV, a second-order damage tensor, is introduced to describe the anisotropic damage state of the carbon nanotube composite so as to inform the continuum macroscale model for composites that are used for SWCNT-PE studies to 
predict fracture. Also, PUCNT is considered as a new material that may have more applications in electron transport and improved properties in composite. For future work, the five different structures can be used for studying the electronic transport and improve fracture strength and toughness in PUCNT - PE composite. Our study of PUCNTs is just the beginning of using methods such as molecular dynamics with force fields such as MEAM-BO* to understand these interesting nanosystems. 


\section{REFERENCES}

(1) Edison, T. PATENTED JULY S1; 1906. T. A. EDISON. ALKALINB BATTERY. APPLICATION FILED VI'LY 21,71904; US Patent 4709251892.

(2) Zhang, Q. L.; O’Brien, S. C.; Heath, J. R.; Liu, Y.; Curl, R. F.; Kroto, H. W.; Smalley, R. E. Reactivity of Large Carbon Clusters: Spheroidal Carbon Shells and Their Possible Relevance to the Formation and Morphology of Soot. J. Phys. Chem. 1986, 90 (4), 525528. https://doi.org/10.1021/j100276a001.

(3) Saito, R.; Dresselhaus, G.; Dresselhaus, M. S. Physical Properties of Carbon Nanotubes; PUBLISHED BY IMPERIAL COLLEGE PRESS AND DISTRIBUTED BY WORLD SCIENTIFIC PUBLISHING CO., 1998. https://doi.org/10.1142/p080.

(4) Iijima, S. Helical Microtubules of Graphitic Carbon. Nature 1991, 354, 56.

(5) Gregory M., O.; Vasyl M., H.; Kristopher E., W.; Thomas S., G. Constitutive Modeling of Nanotube-Reinforced Polymer Composite Systems; NASA Langley Technical Report Server, 2001.

(6) Costello, G. A.; Bert, C. W. Theory of Wire Rope. J. Appl. Mech. 1992, 59 (2), 469. https://doi.org/10.1115/1.2899552.

(7) Thess, A.; Lee, R.; Nikolaev, P.; Robert, J.; Lee, Y. H.; Kim, S. G.; Rinzler, A.; Colbert, D.; Scuseria, G. E.; Fischer, J. E.; Smalley, R. Crystalline Ropes of Metallic Carbon Nanotubes. 1996, 273, 5.

(8) Dai, H.; Rinzler, A. G.; Nikolaev, P.; Thess, A.; Colbert, D. T.; Smalley, R. E. SingleWall Nanotubes Produced by Metal-Catalyzed Disproportionation of Carbon Monoxide. Chem. Phys. Lett. 1996, 260 (3-4), 471-475. https://doi.org/10.1016/00092614(96)00862-7.

(9) Dai, H. Controlling Nanotube Growth. Phys. World 2000, 13 (6), 43-48. https://doi.org/10.1088/2058-7058/13/6/28.

(10) Baughman, R. H. Carbon Nanotubes--the Route Toward Applications. Science 2002, 297 (5582), 787-792. https://doi.org/10.1126/science.1060928.

(11) Androulidakis, Ch.; Tsoukleri, G.; Koutroumanis, N.; Gkikas, G.; Pappas, P.; Parthenios, J.; Papagelis, K.; Galiotis, C. Experimentally Derived Axial Stress-Strain Relations for Two-Dimensional Materials Such as Monolayer Graphene. Carbon 2015, 81, 322-328. https://doi.org/10.1016/j.carbon.2014.09.064. 
(12) Liu, M.; Papageorgiou, D. G.; Li, S.; Lin, K.; Kinloch, I. A.; Young, R. J. Micromechanics of Reinforcement of a Graphene-Based Thermoplastic Elastomer Nanocomposite. Compos. Part Appl. Sci. Manuf. 2018, 110, 84-92. https://doi.org/10.1016/j.compositesa.2018.04.014.

(13) Falkovsky, L. A. Optical Properties of Graphene. J. Phys. 2008, 8.

(14) Soldano, C.; Mahmood, A.; Dujardin, E. Production, Properties and Potential of Graphene. 2010, 24.

(15) Jauregui, L. A.; Yue, Y.; Sidorov, A. N.; Hu, J.; Yu, Q.; Lopez, G.; Jalilian, R.; Benjamin, D. K.; Delkd, D. A.; Wu, W.; Liu, Z.; Wang, X.; Jiang, Z.; Ruan, X.; Bao, J.; Pei, S. S.; Chen, Y. P. Thermal Transport in Graphene Nanostructures: Experiments and Simulations. ECS Trans. 2019, 28 (5), 73-83. https://doi.org/10.1149/1.3367938.

(16) Abergel, D. S. L.; Apalkov, V.; Berashevich, J.; Ziegler, K.; Chakraborty, T. Properties of Graphene: A Theoretical Perspective. Adv. Phys. 2010, 59 (4), 261-482. https://doi.org/10.1080/00018732.2010.487978.

(17) Neto, A. H. C. The Electronic Properties of Graphene. Rev Mod Phys 2009, 81 (1), 54.

(18) Rafii-Tabar, H. Computational Physics of Carbon Nanotubes.; Cambridge University Press, 2008.

(19) Busetto, A. Computational Methods for Carbon Nanotube, università degli studi di padova, 2006.

(20) Gou, J.; Liang, Z.; Zhang, C.; Wang, B. Computational Analysis of Effect of SingleWalled Carbon Nanotube Rope on Molecular Interaction and Load Transfer of Nanocomposites. Compos. Part B Eng. 2005, 36 (6-7), 524-533. https://doi.org/10.1016/j.compositesb.2005.02.004.

(21) Guo, T.; Nikolaev, P.; Thess, A.; Colbert, D. T.; Smalley, R. E. Catalytic Growth of Single-Walled Nanotubes by Laser Vaporization. Chem. Phys. Lett. 1995, 6.

(22) Walters, D. A.; Ericson, L. M.; Casavant, M. J.; Liu, J.; Colbert, D. T.; Smith, K. A.; Smalley, R. E. Elastic Strain of Freely Suspended Single-Wall Carbon Nanotube Ropes. Appl. Phys. Lett. 1999, 74 (25), 3803-3805. https://doi.org/10.1063/1.124185.

(23) Iijima, S.; Ajayan, P. M.; Ichihashi, T. Growth Model for Carbon Nanotubes. Phys. Rev. Lett. 1992, 69 (21), 3100-3103. https://doi.org/10.1103/PhysRevLett.69.3100.

(24) Cassell, A. M.; Raymakers, J. A.; Kong, J.; Dai, H. Large Scale CVD Synthesis of SingleWalled Carbon Nanotubes. J. Phys. Chem. B 1999, 103 (31), 6484-6492. https://doi.org/10.1021/jp990957s. 
(25) Karthikeyan, S.; Mahalingam, P.; Karthik, M. Large Scale Synthesis of Carbon Nanotubes. E-J. Chem. 2009, 6 (1), 1-12. https://doi.org/10.1155/2009/756410.

(26) $\mathrm{Yu}, \mathrm{M}$. Strength and Breaking Mechanism of Multiwalled Carbon Nanotubes Under Tensile Load. Science 2000, 287 (5453), 637-640. https://doi.org/10.1126/science.287.5453.637.

(27) Kong, J.; Soh, H. T.; Cassell, A. M.; Quate, C. F.; Dai, H. Synthesis of Individual SingleWalled Carbon Nanotubes on Patterned Silicon Wafers. Nature 1998, 395 (6705), 878881. https://doi.org/10.1038/27632.

(28) Pan, Z. W.; Xie, S. S.; Lu, L.; Chang, B. H.; Sun, L. F.; Zhou, W. Y.; Wang, G.; Zhang, D. L. Tensile Tests of Ropes of Very Long Aligned Multiwall Carbon Nanotubes. Appl. Phys. Lett. 1999, 74 (21), 3152-3154. https://doi.org/10.1063/1.124094.

(29) Yu, M.-F.; Files, B. S.; Arepalli, S.; Ruoff, R. S. Tensile Loading of Ropes of Single Wall Carbon Nanotubes and Their Mechanical Properties. Phys. Rev. Lett. 2000, 84 (24), $5552-$ 5555. https://doi.org/10.1103/PhysRevLett.84.5552.

(30) Kuzmany, H.; Burger, B.; Hulman, M.; Kürti, J.; Rinzler, A. G.; Smalley, R. E. Spectroscopic Analysis of Different Types of Single-Wall Carbon Nanotubes. Europhys. Lett. EPL 1998, 44 (4), 518-524. https://doi.org/10.1209/epl/i1998-00504-y.

(31) Rao, A. M. Diameter-Selective Raman Scattering from Vibrational Modes in Carbon Nanotubes. Science 1997, 275 (5297), 187-191. https://doi.org/10.1126/science.275.5297.187.

(32) Yakobson, B. I.; Campbell, M. P.; Brabec, C. J.; Bernholc, J. High Strain Rate Fracture and C-Chain Unraveling in Carbon Nanotubes. Comput. Mater. Sci. 1997, 8.

(33) Buongiorno Nardelli, M.; Yakobson, B. I.; Bernholc, J. Mechanism of Strain Release in Carbon Nanotubes. Phys. Rev. B 1998, 57 (8), R4277-R4280.

https://doi.org/10.1103/PhysRevB.57.R4277.

(34) Yakobson, B. I.; Avouris, P. Mechanical Properties of Carbon Nanotubes. In Carbon Nanotubes; Dresselhaus, M. S., Dresselhaus, G., Avouris, P., Eds.; Springer Berlin Heidelberg: Berlin, Heidelberg, 2001; Vol. 80, pp 287-327. https://doi.org/10.1007/3-54039947-X_12.

(35) Amara, H.; Bichara, C. Modeling the Growth of Single-Wall Carbon Nanotubes. Top. Curr. Chem. 2017, 375 (3), 55. https://doi.org/10.1007/s41061-017-0141-8.

(36) Yakobson, B. I.; Brabec, C. J.; Bernholc, J. Nanomechanics of Carbon Tubes: Instabilities beyond Linear Response. Phys. Rev. Lett. 1996, 76 (14), 2511-2514. https://doi.org/10.1103/PhysRevLett.76.2511. 
(37) Kürti, J.; Kresse, G.; Kuzmany, H. First-Principles Calculations of the Radial Breathing Mode of Single-Wall Carbon Nanotubes. Phys. Rev. B 1998, 58 (14), R8869-R8872. https://doi.org/10.1103/PhysRevB.58.R8869.

(38) Kürti, J.; Zólyomi, V.; Kertesz, M.; Guangyu, S. The Geometry and the Radial Breathing Mode of Carbon Nanotubes: Beyond the Ideal Behaviour. New J. Phys. 2003, 5, 125-125. https://doi.org/10.1088/1367-2630/5/1/125.

(39) Ghavanloo, E.; Fazelzadeh, S. A. Vibration Characteristics of Single-Walled Carbon Nanotubes Based on an Anisotropic Elastic Shell Model Including Chirality Effect. Appl. Math. Model. 2012, 36 (10), 4988-5000. https://doi.org/10.1016/j.apm.2011.12.036.

(40) Lawler, H. M.; Areshkin, D.; Mintmire, J. W.; White, C. T. Radial-Breathing Mode Frequencies for Single-Walled Carbon Nanotubes of Arbitrary Chirality: First-Principles Calculations. Phys. Rev. B 2005, 72 (23), 233403.

https://doi.org/10.1103/PhysRevB.72.233403.

(41) Nouri, N.; Ziaei-Rad, S.; Adibi, S.; Karimzadeh, F. Fabrication and Mechanical Property Prediction of Carbon Nanotube Reinforced Aluminum Nanocomposites. Mater. Des. 2012, 34, 1-14. https://doi.org/10.1016/j.matdes.2011.07.047.

(42) Karimzadeh, F.; Ziaei-Rad, S.; Adibi, S. Modeling Considerations and Material Properties Evaluation in Analysis of Carbon Nano-Tubes Composite. Metall. Mater. Trans. B 2007, 38 (4), 695-705. https://doi.org/10.1007/s11663-007-9065-y.

(43) Strano, M. S.; Doorn, S. K.; Haroz, E. H.; Kittrell, C.; Hauge, R. H.; Smalley, R. E. Assignment of $(n, m)$ Raman and Optical Features of Metallic Single-Walled Carbon Nanotubes. Nano Lett. 2003, 3 (8), 1091-1096. https://doi.org/10.1021/n1034196n.

(44) Kasuya, A.; Sasaki, Y.; Saito, Y.; Tohji, K.; Nishina, Y. Evidence for Size-Dependent Discrete Dispersions in Single-Wall Nanotubes. Phys. Rev. Lett. 1997, 78 (23), 44344437. https://doi.org/10.1103/PhysRevLett.78.4434.

(45) Venkateswaran, U. D.; Rao, A. M.; Richter, E.; Menon, M.; Rinzler, A.; Smalley, R. E.; Eklund, P. C. Probing the Single-Wall Carbon Nanotube Bundle: Raman Scattering under High Pressure. Phys. Rev. B 1999, 59 (16), 10928-10934.

https://doi.org/10.1103/PhysRevB.59.10928.

(46) Kasuya, A.; Sugano, M.; Maeda, T.; Saito, Y.; Tohji, K.; Takahashi, H.; Sasaki, Y.; Fukushima, M.; Nishina, Y.; Horie, C. Resonant Raman Scattering and the Zone-Folded Electronic Structure in Single-Wall Nanotubes. Phys. Rev. B 1998, 57 (9), 4999-5001. https://doi.org/10.1103/PhysRevB.57.4999.

(47) Sánchez-Portal, D.; Artacho, E.; Soler, J. M.; Rubio, A.; Ordejón, P. Ab Initio Structural, Elastic, and Vibrational Properties of Carbon Nanotubes. Phys. Rev. B 1999, 59 (19), 12678-12688. https://doi.org/10.1103/PhysRevB.59.12678. 
(48) Maeda, T.; Horie, C. Phonon Modes in Single-Wall Nanotubes with a Small Diameter. Phys. B Condens. Matter 1999, 263-264, 479-481. https://doi.org/10.1016/S09214526(98)01415-X.

(49) Yu, J.; Kalia, R. K.; Vashishta, P. Phonons in Graphitic Tubules: A Tight-binding Molecular Dynamics Study. J. Chem. Phys. 1995, 103 (15), 6697-6705. https://doi.org/10.1063/1.470400.

(50) Saito, R.; Takeya, T.; Kimura, T.; Dresselhaus, G.; Dresselhaus, M. S. Raman Intensity of Single-Wall Carbon Nanotubes. Phys. Rev. B 1998, 57 (7), 4145-4153. https://doi.org/10.1103/PhysRevB.57.4145.

(51) Henrard, L.; Hernández, E.; Bernier, P.; Rubio, A. Van Der Waals Interaction in Nanotube Bundles: Consequences on Vibrational Modes. Phys. Rev. B 1999, 60 (12), R8521-R8524. https://doi.org/10.1103/PhysRevB.60.R8521.

(52) Longhurst, M. J.; Quirke, N. The Radial Breathing Mode of Carbon Nanotubes. Mol. Simul. 2005, 31 (2-3), 135-141. https://doi.org/10.1080/08927020412331308520.

(53) Maultzsch, J.; Telg, H.; Reich, S.; Thomsen, C. Radial Breathing Mode of Single-Walled Carbon Nanotubes: Optical Transition Energies and Chiral-Index Assignment. Phys. Rev. B 2005, 72 (20), 205438. https://doi.org/10.1103/PhysRevB.72.205438.

(54) Pine, P.; Yaish, Y. E.; Adler, J. Simulation and Vibrational Analysis of Thermal Oscillations of Single-Walled Carbon Nanotubes. Phys. Rev. B 2011, 83 (15), 155410. https://doi.org/10.1103/PhysRevB.83.155410.

(55) Tailor, P. M.; Wheatley, R. J.; Besley, N. A. An Empirical Force Field for the Simulation of the Vibrational Spectroscopy of Carbon Nanomaterials. Carbon 2017, 113, 299-308. https://doi.org/10.1016/j.carbon.2016.11.059.

(56) Jorio, A.; Fantini, C.; Pimenta, M. A.; Capaz, R. B.; Samsonidze, Ge. G.; Dresselhaus, G.; Dresselhaus, M. S.; Jiang, J.; Kobayashi, N.; Grüneis, A.; Saito, R. Resonance Raman Spectroscopy ( n , m ) -Dependent Effects in Small-Diameter Single-Wall Carbon Nanotubes. Phys. Rev. B 2005, 71 (7), 075401. https://doi.org/10.1103/PhysRevB.71.075401.

(57) Kürti, J. Theoretical Investigation of Small Diameter Single-Wall Carbon Nanotubes. In AIP Conference Proceedings; AIP: Kirchberg, Tirol (AUSTRIA), 2003; Vol. 685, pp 456-459. https://doi.org/10.1063/1.1628071.

(58) Popov, V. N.; Lambin, P. Radius and Chirality Dependence of the Radial Breathing Mode and the G -Band Phonon Modes of Single-Walled Carbon Nanotubes. Phys. Rev. B 2006, 73 (8), 085407. https://doi.org/10.1103/PhysRevB.73.085407. 
(59) Telg, H.; Maultzsch, J.; Reich, S.; Hennrich, F.; Thomsen, C. Chirality Distribution and Transition Energies of Carbon Nanotubes. Phys. Rev. Lett. 2004, 93 (17), 177401. https://doi.org/10.1103/PhysRevLett.93.177401.

(60) Meyer, J. C.; Paillet, M.; Michel, T.; Moréac, A.; Neumann, A.; Duesberg, G. S.; Roth, S.; Sauvajol, J.-L. Raman Modes of Index-Identified Freestanding Single-Walled Carbon Nanotubes. Phys. Rev. Lett. 2005, 95 (21), 217401. https://doi.org/10.1103/PhysRevLett.95.217401.

(61) Chang, T. Radial Breathing Mode Frequency of Single-Walled Carbon Nanotubes under Strain. Appl. Phys. Lett. 2008, 93 (6), 061901. https://doi.org/10.1063/1.2969033.

(62) Damnjanović, M.; Dobardžić, E.; Milošević, I. Chirality Dependence of the Radial Breathing Mode: A Simple Model. J. Phys. Condens. Matter 2004, 16 (49), L505-L508. https://doi.org/10.1088/0953-8984/16/49/L02.

(63) Bandow, S.; Asaka, S.; Saito, Y.; Rao, A. M.; Grigorian, L.; Richter, E.; Eklund, P. C. Effect of the Growth Temperature on the Diameter Distribution and Chirality of SingleWall Carbon Nanotubes. Phys. Rev. Lett. 1998, 80 (17), 3779-3782. https://doi.org/10.1103/PhysRevLett.80.3779.

(64) Alvarez, L.; Righi, A.; Guillard, T.; Rols, S.; Anglaret, E.; Laplaze, D.; Sauvajol, J.-L. Resonant Raman Study of the Structure and Electronic Properties of Single-Wall Carbon Nanotubes. Chem. Phys. Lett. 2000, 316 (3-4), 186-190. https://doi.org/10.1016/S00092614(99)01291-9.

(65) On the Determination of Molecular Fields. - II. From the Equation of State of a Gas. Proc. R. Soc. Lond. Ser. Contain. Pap. Math. Phys. Character 1924, 106 (738), 463-477. https://doi.org/10.1098/rspa.1924.0082.

(66) Jishi, R. A.; Venkataraman, L.; Dresselhaus, M. S.; Dresselhaus, G. Phonon Modes in Carbon Nanotubules. Chem. Phys. Lett. 1993, 209 (1-2), 77-82. https://doi.org/10.1016/0009-2614(93)87205-H.

(67) Treacy, M. M. J.; Ebbesen, T. W.; Gibson, J. M. Exceptionally High Young's Modulus Observed for Individual Carbon Nanotubes. Nature 1996, 381 (6584), 678-680. https://doi.org/10.1038/381678a0.

(68) Overney, G.; Zhong, W.; Tomnek, D. Structural Rigidity and Low Frequency Vibrational Modes of Long Carbon Tubules. Z. Fr Phys. At. Mol. Clust. 1993, 27 (1), 93-96. https://doi.org/10.1007/BF01436769.

(69) Hernández, E.; Goze, C.; Bernier, P.; Rubio, A. Elastic Properties of C and B x C y N z Composite Nanotubes. Phys. Rev. Lett. 1998, 80 (20), 4502-4505. https://doi.org/10.1103/PhysRevLett.80.4502. 
(70) Lu, J. P. Elastic Properties of Carbon Nanotubes and Nanoropes. Phys. Rev. Lett. 1997, 79 (7), 4.

(71) Robertson, D. H.; Brenner, D. W.; Mintmire, J. W. Energetics of Nanoscale Graphitic Tubules. Phys. Rev. B 1992, 45 (21), 12592-12595. https://doi.org/10.1103/PhysRevB.45.12592.

(72) Yakobson, B. I.; Brabec, C. J.; Bernholc, J. Structural Mechanics of Carbon Nanotubes: From Continuum Elasticity to Atomistic Fracture. J. Comput.-Aided Mater. Des. 1996, 3 (1-3), 173-182. https://doi.org/10.1007/BF01185652.

(73) Arruda, E.; Boyce, M. EFFECTS OF INITIAL ANISOTROPY ON THE FINITE STRAIN DEFORMATION BEHAVIOR OF GLASSY POLYMERS. 29.

(74) Tersoff, J. New Empirical Approach for the Structure and Energy of Covalent Systems. Phys. Rev. B 1988, 37 (12), 6991-7000. https://doi.org/10.1103/PhysRevB.37.6991.

(75) Brenner, D. W. Empirical Potential for Hydrocarbons for Use in Simulating the Chemical Vapor Deposition of Diamond Films. Phys. Rev. B 1990, 42 (15), 9458-9471. https://doi.org/10.1103/PhysRevB.42.9458.

(76) Kanamitsu, K.; Saito, S. Geometries, Electronic Properties, and Energetics of Isolated Single Walled Carbon Nanotubes. J. Phys. Soc. Jpn. 2002, 71 (2), 483-486. https://doi.org/10.1143/JPSJ.71.483.

(77) Kamimura, H. ENERGETICS OF CARBON NANO-TUBES Shin-Ichi Sawada and Noriaki Hamada. 3.

(78) Nielson, K. D.; van Duin, A. C. T.; Oxgaard, J.; Deng, W.-Q.; Goddard, W. A. Development of the ReaxFF Reactive Force Field for Describing Transition Metal Catalyzed Reactions, with Application to the Initial Stages of the Catalytic Formation of Carbon Nanotubes. J. Phys. Chem. A 2005, 109 (3), 493-499. https://doi.org/10.1021/jp046244d.

(79) Mun, S.; Bowman, A. L.; Nouranian, S.; Gwaltney, S. R.; Baskes, M. I.; Horstemeyer, M. F. Interatomic Potential for Hydrocarbons on the Basis of the Modified Embedded-Atom Method with Bond Order (MEAM-BO). J. Phys. Chem. A 2017, 121 (7), 1502-1524. https://doi.org/10.1021/acs.jpca.6b11343.

(80) Brenner, D. W.; Shenderova, O. A.; Harrison, J. A.; Stuart, S. J.; Ni, B.; Sinnott, S. B. A Second-Generation Reactive Empirical Bond Order (REBO) Potential Energy Expression for Hydrocarbons. J. Phys. Condens. Matter 2002, 14 (4), 783-802. https://doi.org/10.1088/0953-8984/14/4/312.

(81) Srinivasan, S. G.; van Duin, A. C. T.; Ganesh, P. Development of a ReaxFF Potential for Carbon Condensed Phases and Its Application to the Thermal Fragmentation of a Large Fullerene. J. Phys. Chem. A 2015, 119 (4), 571-580. https://doi.org/10.1021/jp510274e. 
(82) Daw, M. S.; Baskes, M. I. Embedded-Atom Method: Derivation and Application to Impurities, Surfaces, and Other Defects in Metals. Phys. Rev. B 1984, 29 (12), 6443-6453. https://doi.org/10.1103/PhysRevB.29.6443.

(83) Gall, K.; Horstemeyer, M. F.; Van Schilfgaarde, M.; Baskes, M. I. Atomistic Simulations on the Tensile Debonding of an Aluminum-Silicon Interface. J. Mech. Phys. Solids 2000, 48 (10), 2183-2212. https://doi.org/10.1016/S0022-5096(99)00086-1.

(84) Potirniche, G. P.; Horstemeyer, M. F.; Wagner, G. J.; Gullett, P. M. A Molecular Dynamics Study of Void Growth and Coalescence in Single Crystal Nickel. Int. J. Plast. 2006, 22 (2), 257-278. https://doi.org/10.1016/j.ijplas.2005.02.001.

(85) Nouranian, S.; Tschopp, M. A.; Gwaltney, S. R.; Baskes, M. I.; Horstemeyer, M. F. An Interatomic Potential for Saturated Hydrocarbons Based on the Modified Embedded-Atom Method. Phys Chem Chem Phys 2014, 16 (13), 6233-6249. https://doi.org/10.1039/C4CP00027G.

(86) Potirniche, G. P.; Horstemeyer, M. F.; Gullett, P. M.; Jelinek, B. Atomistic Modelling of Fatigue Crack Growth and Dislocation Structuring in FCC Crystals. Proc. R. Soc. Math. Phys. Eng. Sci. 2006, 462 (2076), 3707-3731. https://doi.org/10.1098/rspa.2006.1746.

(87) Horstemeyer, M. F.; Farkas, D.; Kim, S.; Tang, T.; Potirniche, G. Nanostructurally Small Cracks (NSC): A Review on Atomistic Modeling of Fatigue. Int. J. Fatigue 2010, 32 (9), 1473-1502. https://doi.org/10.1016/j.ijfatigue.2010.01.006.

(88) Huddleston, B. D.; Dickel, D. E.; Williams, N.; Danielson, K.; Hammi, Y.; Bowman, A. L.; Baskes, M. I.; Horstemeyer, M. F. Correlating Damage Progression to Fragmentation at High Strain Rates Using Molecular Dynamics. Model. Simul. Mater. Sci. Eng. 2020, 28 (2), 025009. https://doi.org/10.1088/1361-651X/ab6364.

(89) Nouranian, S.; Gwaltney, S. R.; Baskes, M. I.; Tschopp, M. A.; Horstemeyer, M. F. Simulations of Tensile Bond Rupture in Single Alkane Molecules Using Reactive Interatomic Potentials. Chem. Phys. Lett. 2015, 635, 278-284. https://doi.org/10.1016/j.cplett.2015.06.071.

(90) Vinet, P.; Ferrante, J.; Smith, J. R.; Rose, J. H. A Universal Equation of State for Solids. J. Phys. C Solid State Phys. 1986, 19 (20), L467-L473. https://doi.org/10.1088/00223719/19/20/001.

(91) Dickel, D.; Gwaltney, S. R.; Mun, S.; Baskes, M. I.; Horstemeyer, M. F. DispersionCorrected Modified Embedded-Atom Method Bond Order Interatomic Potential for Sulfur. J. Phys. Chem. A 2018, 122 (49), 9572-9578. https://doi.org/10.1021/acs.jpca.8b07410.

Mathworks. MATLAB; www.mathworks.com, 2017. 
(93) Gao, Y.; Wang, H.; Daw, M. S. Calculations of Lattice Vibrational Mode Lifetimes Using Jazz: A Python Wrapper for LAMMPS. Model. Simul. Mater. Sci. Eng. 2015, 23 (4), 045002. https://doi.org/10.1088/0965-0393/23/4/045002.

(94) Plimpton, S.; Crozier, P.; Thompson, A. LAMMPS-Large-Scale Atomic/Molecular Massively Parallel Simulator. Sandia Natl. Lab. 2007, 18, 43.

(95) Alford, J. T.; Landis, B. A.; Mintmire, J. W. Theoretical Elastic Properties of SingleWalled Carbon Nanotubes. Int. J. Quantum Chem. 2005, 105 (6), 767-771. https://doi.org/10.1002/qua.20660.

(96) Byron Pipes, R.; Frankland, S. J. V.; Hubert, P.; Saether, E. Self-Consistent Properties of Carbon Nanotubes and Hexagonal Arrays as Composite Reinforcements. Compos. Sci. Technol. 2003, 63 (10), 1349-1358. https://doi.org/10.1016/S0266-3538(03)00020-4.

(97) Cheng, Y.; Zhang, S.; Li, J.; Sun, J.; Wang, J.; Qin, C.; Dai, L. Preparation of Functionalized Partially Unzipped Carbon Nanotube/Polyimide Composite Fibers with Increased Mechanical and Thermal Properties. RSC Adv. 2017, 7 (35), 21953-21961. https://doi.org/10.1039/C7RA01738C.

(98) Zhao, Q.; Gan, X.; Lei, Q.; Li, W.; Zhou, K. Enhanced the Strength and Ductility of the Partially Unzipped Carbon Nanotubes Reinforced CuCr Matrix Composites via Optimization of the Interface Structure. Compos. Interfaces 2020, 1-11. https://doi.org/10.1080/09276440.2019.1707026.

(99) Kosynkin, D. V.; Higginbotham, A. L.; Sinitskii, A.; Lomeda, J. R.; Dimiev, A.; Price, B. K.; Tour, J. M. Longitudinal Unzipping of Carbon Nanotubes to Form Graphene Nanoribbons. Nature 2009, 458 (7240), 872-876. https://doi.org/10.1038/nature07872.

(100) Elías, A. L.; Botello-Méndez, A. R.; Meneses-Rodríguez, D.; Jehová González, V.; Ramírez-González, D.; Ci, L.; Muñoz-Sandoval, E.; Ajayan, P. M.; Terrones, H.; Terrones, M. Longitudinal Cutting of Pure and Doped Carbon Nanotubes to Form Graphitic Nanoribbons Using Metal Clusters as Nanoscalpels. Nano Lett. 2010, 10 (2), 366-372. https://doi.org/10.1021/n1901631z.

(101) Jiao, L.; Zhang, L.; Wang, X.; Diankov, G.; Dai, H. Narrow Graphene Nanoribbons from Carbon Nanotubes. Nature 2009, 458 (7240), 877-880.

https://doi.org/10.1038/nature07919.

(102) Cano-Márquez, A. G.; Rodríguez-Macías, F. J.; Campos-Delgado, J.; Espinosa-González, C. G.; Tristán-López, F.; Ramírez-González, D.; Cullen, D. A.; Smith, D. J.; Terrones, M.; Vega-Cantú, Y. I. Ex-MWNTs: Graphene Sheets and Ribbons Produced by Lithium Intercalation and Exfoliation of Carbon Nanotubes. Nano Lett. 2009, 9 (4), 1527-1533. https://doi.org/10.1021/nl803585s. 
(103) Cano-Márquez, A. G.; Rodríguez-Macías, F. J.; Campos-Delgado, J.; Espinosa-González, C. G.; Tristán-López, F.; Ramírez-González, D.; Cullen, D. A.; Smith, D. J.; Terrones, M.; Vega-Cantú, Y. I. Ex-MWNTs: Graphene Sheets and Ribbons Produced by Lithium Intercalation and Exfoliation of Carbon Nanotubes. Nano Lett. 2009, 9 (4), 1527-1533. https://doi.org/10.1021/n1803585s.

(104) Rangel, N. L.; Sotelo, J. C.; Seminario, J. M. Mechanism of Carbon Nanotubes Unzipping into Graphene Ribbons. J. Chem. Phys. 2009, 131 (3), 031105. https://doi.org/10.1063/1.3170926.

(105) Huang, B.; Son, Y.-W.; Kim, G.; Duan, W.; Ihm, J. Electronic and Magnetic Properties of Partially Open Carbon Nanotubes. J. Am. Chem. Soc. 2009, 131 (49), 17919-17925. https://doi.org/10.1021/ja907212g.

(106) Costamagna, S.; Schulz, A.; Covaci, L.; Peeters, F. Partially Unzipped Carbon Nanotubes as Magnetic Field Sensors. Appl. Phys. Lett. 2012, 100 (23), 232104. https://doi.org/10.1063/1.4726039.

(107) Chen, X.; Xu, Y.; Zou, X.; Gu, B.-L.; Duan, W. Interfacial Thermal Conductance of Partially Unzipped Carbon Nanotubes: Linear Scaling and Exponential Decay. Phys. Rev. $B$ 2013, 87 (15), 155438. https://doi.org/10.1103/PhysRevB.87.155438.

(108) Tang, C.; Guo, W.; Chen, C. Structural and Mechanical Properties of Partially Unzipped Carbon Nanotubes. Phys. Rev. B 2011, 83 (7), 075410. https://doi.org/10.1103/PhysRevB.83.075410.

(109) Ni, X.; Zhang, G.; Li, B. Thermal Conductivity and Thermal Rectification in Unzipped Carbon Nanotubes. J. Phys. Condens. Matter 2011, 23 (21), 215301. https://doi.org/10.1088/0953-8984/23/21/215301.

(110) Novotny, M. A.; Novotný, T. Order amidst Disorder in 2D+3D Quantum Dragon Composite Nanodevices with Varying Breadth. J. Phys. Conf. Ser. 2021, 1740, 012002. https://doi.org/10.1088/1742-6596/1740/1/012002.

(111) Tang, C.; Ishihara, H.; Sodhi, J.; Chen, Y.-C.; Siordia, A.; Martini, A.; Tung, V. C. Flexible All-Carbon Photovoltaics with Improved Thermal Stability. J. Solid State Chem. 2015, 224, 94-101. https://doi.org/10.1016/j.jssc.2014.07.010.

(112) Stuart, S. J.; Tutein, A. B.; Harrison, J. A. A Reactive Potential for Hydrocarbons with Intermolecular Interactions. J. Chem. Phys. 2000, 112 (14), 6472-6486. https://doi.org/10.1063/1.481208.

(113) Humphrey, W.; Dalke, A.; Schulten, K. VMD: Visual Molecular Dynamics. J. Mol. Graph. 1996, 14 (1), 33-38. https://doi.org/10.1016/0263-7855(96)00018-5.

(114) Materials and Processes Simulations(MAPS); Scienomics SARL, 2004-2018, 2018. 
(115) Singh, A.; Kumar, D. Effect of Temperature on Elastic Properties of CNT-Polyethylene Nanocomposite and Its Interface Using MD Simulations. J. Mol. Model. 2018, 24 (7), 178. https://doi.org/10.1007/s00894-018-3716-6.

(116) Dereli, G.; Süngü, B. Temperature Dependence of the Tensile Properties of Single-Walled Carbon Nanotubes: O ( N ) Tight-Binding Molecular-Dynamics Simulations. Phys. Rev. B 2007, 75 (18), 184104. https://doi.org/10.1103/PhysRevB.75.184104.

(117) Kundalwal, S. I. Multiscale Modeling of Regularly Staggered Carbon Fibers Embedded in Nano-Reinforced Composites. 2017, 16.

(118) Xiong, Q.; Tian, X. Atomistic Modeling of Mechanical Characteristics of CNTPolyethylene with Interfacial Covalent Interaction. J. Nanomater. 2015, 2015, 1-9. https://doi.org/10.1155/2015/237520.

(119) Ghosh, M.; Maiti, S.; Dutta, S.; Das, D.; Das, P. K. Covalently Functionalized SingleWalled Carbon Nanotubes at Reverse Micellar Interface: A Strategy to Improve Lipase Activity. 2012, 10.

(120) Huang, Z. Covalent Functionalization of Multi-Walled Carbon Nanotubes with Quaternary Ammonium Groups and Its Application in Ion Chromatography. 2013, 8.

(121) Vigolo, B.; Mamane, V.; Valsaque, F.; Le, T. N. H.; Thabit, J.; Ghanbaja, J.; Aranda, L.; Fort, Y.; McRae, E. Evidence of Sidewall Covalent Functionalization of Single-Walled Carbon Nanotubes and Its Advantages for Composite Processing. Carbon 2009, 47 (2), 411-419. https://doi.org/10.1016/j.carbon.2008.10.024.

(122) Fifield, L. S.; Grate, J. W. Hydrogen-Bond Acidic Functionalized Carbon Nanotubes (CNTs) with Covalently-Bound Hexafluoroisopropanol Groups. 2010, 4.

(123) Hu, H.; Yu, B.; Ye, Q.; Gu, Y.; Zhou, F. Modification of Carbon Nanotubes with a Nanothin Polydopamine Layer and Polydimethylamino-Ethyl Methacrylate Brushes. 2010, 7.

(124) Peyvandi, A. Surface-Modified Graphite Nanomaterials for Improved Reinforcement Efficiency in Cementitious Paste. 2013, 12.

(125) Gorrasi, G.; Di Lieto, R.; Patimo, G.; De Pasquale, S.; Sorrentino, A. Structure-Property Relationships on Uniaxially Oriented Carbon Nanotube/Polyethylene Composites. Polymer 2011, 52 (4), 1124-1132. https://doi.org/10.1016/j.polymer.2011.01.008.

(126) Pham, J. Q.; Mitchell, C. A.; Bahr, J. L.; Tour, J. M.; Krishanamoorti, R.; Green, P. F. Glass Transition of Polymer/Single-Walled Carbon Nanotube Composite Films. J. Polym. Sci. Part B Polym. Phys. 2003, 41 (24), 3339-3345. https://doi.org/10.1002/polb.10702. 
(127) Wang, J.; Cao, C.; Chen, X.; Ren, S.; Chen, Y.; Yu, D.; Chen, X. Orientation and Dispersion Evolution of Carbon Nanotubes in Ultra High Molecular Weight Polyethylene Composites under Extensional-Shear Coupled Flow: A Dissipative Particle Dynamics Study. Polymers 2019, 11 (1), 154. https://doi.org/10.3390/polym11010154.

(128) Penumadu, D.; Dutta, A.; Pharr, G. M.; Files, B. Mechanical Properties of Blended SingleWall Carbon Nanotube Composites. J. Mater. Res. 2003, 18 (8), 1849-1853. https://doi.org/10.1557/JMR.2003.0258.

(129) Bryan R. Loyola; Kenneth J. Loh; Valeria La Saponara. Static and Dynamic Strain Monitoring of GFRP Composites Using Carbon Nanotube Thin Films; 2011; Vol. 7981. https://doi.org/10.1117/12.881006.

(130) Srivastava and, D.; Wei, C.; Cho, K. Nanomechanics of Carbon Nanotubes and Composites. Appl. Mech. Rev. 2003, 56 (2), 215-230. https://doi.org/10.1115/1.1538625.

(131) Frankland, S. J. V.; Caglar, A.; Brenner, D. W.; Griebel, M. Molecular Simulation of the Influence of Chemical Cross-Links on the Shear Strength of Carbon Nanotube-Polymer Interfaces. 3.

(132) Tahreen, N.; Masud, A. K. M. Investigation of the Mechanical Properties of Polyethylene/Carbon Nanotube Composite by Molecular Dynamics Simulation. 16.

(133) Liu, L.-Y.; Zhang, Z.-X.; Gou, X.-F.; Yang, H.-X. Molecular Modelling of the Effect of Loading Rate on Elastic Properties of CNT-Polyethylene Nanocomposite and Its Interface. Mater. Res. Express 2020, 6 (12), 1250d2. https://doi.org/10.1088/2053-1591/ab61b2.

(134) Goh, C. S.; Wei, J.; Lee, L. C.; Gupta, M. Development of Novel Carbon Nanotube Reinforced Magnesium Nanocomposites Using the Powder Metallurgy Technique. Nanotechnology 2006, 17 (1), 7-12. https://doi.org/10.1088/0957-4484/17/1/002.

(135) Goh, C. S.; Wei, J.; Lee, L. C.; Gupta, M. Simultaneous Enhancement in Strength and Ductility by Reinforcing Magnesium with Carbon Nanotubes. Mater. Sci. Eng. A 2006, 423 (1-2), 153-156. https://doi.org/10.1016/j.msea.2005.10.071.

(136) Arora, G.; Pathak, H. Experimental and Numerical Approach to Study Mechanical and Fracture Properties of High-Density Polyethylene Carbon Nanotubes Composite. Mater. Today Commun. 2020, 22, 100829. https://doi.org/10.1016/j.mtcomm.2019.100829.

(137) Dasari, A.; Zhang, Q.-X.; Yu, Z.-Z.; Mai, Y.-W. Toughening Polypropylene and Its Nanocomposites with Submicrometer Voids. Macromolecules 2010, 43 (13), 5734-5739. https://doi.org/10.1021/ma100633y.

(138) Bowman, A. L.; Mun, S.; Nouranian, S.; Huddleston, B. D.; Gwaltney, S. R.; Baskes, M. I.; Horstemeyer, M. F. Free Volume and Internal Structural Evolution during Creep in Model Amorphous Polyethylene by Molecular Dynamics Simulations. Polymer 2019, 170, 85-100. https://doi.org/10.1016/j.polymer.2019.02.060. 
(139) Bowman, A. L. Multiscale Damage and Fracture Modeling of Polyethylene Using Molecular Dynamics Simulations and Continuum Internal State Variable Theory. Ph.D., Mississippi State University, Ann Arbor, 2019.

(140) Zhang, Z. Q.; Ward, D. K.; Xue, Y.; Zhang, H. W.; Horstemeyer, M. F. Interfacial Characteristics of Carbon Nanotube-Polyethylene Composites Using Molecular Dynamics Simulations. ISRN Mater. Sci. 2011, 2011, 1-10. https://doi.org/10.5402/2011/145042.

(141) Stukowski, A. Visualization and Analysis of Atomistic Simulation Data with OVITO-the Open Visualization Tool. Model. Simul. Mater. Sci. Eng. 2010, 18 (1), 015012. https://doi.org/10.1088/0965-0393/18/1/015012.

(142) Bažant, Z. P.; Belytschko, T. B.; Chang, T. Continuum Theory for Strain-Softening. J. Eng. Mech. 1984, 110 (12), 1666-1692. https://doi.org/10.1061/(ASCE)07339399(1984)110:12(1666).

(143) Al-Jishi, R.; Dresselhaus, G. Lattice-Dynamical Model for Graphite. Phys. Rev. B 1982, 26 (8), 4514-4522. https://doi.org/10.1103/PhysRevB.26.4514.

(144) Hasegawa, M.; Nishidate, K. Semiempirical Approach to the Energetics of Interlayer Binding in Graphite. Phys. Rev. B 2004, 70 (20), 205431. https://doi.org/10.1103/PhysRevB.70.205431.

(145) Gwaltney, S. R.; Head-Gordon, M. A Second-Order Perturbative Correction to the Coupled-Cluster Singles and Doubles Method: CCSD(2). J. Chem. Phys. 2001, 115 (5), 2014-2021. https://doi.org/10.1063/1.1383589.

(146) Kendall, R. A.; Dunning, T. H., Jr.; Harrison, R. J. Electron Affinities of the First-row Atoms Revisited. Systematic Basis Sets and Wave Functions. J. Chem. Phys. 1992, 96 (9), 6796-6806. https://doi.org/10.1063/1.462569. 
APPENDIX A

GRAPHENE AND GRAPHITE PROPERTIES CALCULATED FROM MEAM-BO** 


\section{A.1 Graphene and graphite properties calculation}

As our previous work ${ }^{79}$ did not explicitly include CNT structure in the fitting database, we performed reparameterizations on the bond order parameters that affect CNT properties only while maintaining other properties as closely as the original. Therefore, the changes in the parameters only affect a few properties related to unsaturated bond cases. The following table shows the comparison between the current work (MEAM-BO*), the previous work (MEAMBO) and the predictions by REBO against graphite and graphene experimental results. While the current MEAM-BO* parameters predict graphene properties similarly to the previous work, the prediction accuracy for graphite properties are greatly improved. Especially, the predicted C44 elastic constant using the updated parameters is much closer to the experimental value, resulting in the significantly improved mean absolute percent (MAP) of 21.7 percent compared to the previously 817 percent. The only drawback in the current parameters is a slightly decreased rotational barrier in the $\mathrm{C}_{2} \mathrm{H}_{4}$ molecule, which is shown in the Figure A.1. 
Table A.1 Graphene and graphite properties calculated from MEAM-BO (previous work ${ }^{79}$ and current work) and REBO against the experimental data. The zero-point energy (ZPE) corrections used here are $0.166 \mathrm{eV} /$ atom for graphene and graphite ${ }^{143,144}$, respectively. $E_{\text {Corr }}$ is the corrected energy by $\mathrm{ZPE}$

\begin{tabular}{|c|c|c|c|c|c|c|c|c|}
\hline \multirow[b]{2}{*}{ Species } & \multirow[b]{2}{*}{ Property } & \multirow[b]{2}{*}{ Expt. } & \multicolumn{2}{|c|}{ MEAMBO* } & \multicolumn{2}{|c|}{ MEAMBO } & \multicolumn{2}{|c|}{ REBO } \\
\hline & & & Calc. & Diff. & Calc. & Diff. & Calc. & Diff. \\
\hline \multirow[t]{8}{*}{ Graphene } & $E_{\text {Corr }}(\mathrm{eV} /$ atom $)$ & $7.315^{\mathrm{b}}$ & 7.303 & -0.012 & 7.304 & -0.011 & 7.243 & -0.072 \\
\hline & Lattice const., a ( & $2.462^{\mathrm{c}}$ & 2.462 & 0 & 2.46 & -0.002 & 2.419 & -0.043 \\
\hline & $C_{11}+C_{12}(\mathrm{~N} / \mathrm{m})$ & $358.1^{\mathrm{d}}$ & 402.3 & 44.2 & 363.4 & 5.3 & 436.9 & 78.8 \\
\hline & $C_{66}(\mathrm{~N} / \mathrm{m})$ & $60.4^{\mathrm{d}}$ & 138.8 & 78.4 & 134.9 & 74.5 & 194.0 & 133.6 \\
\hline & Young's modulus (GPa) & $1020^{\mathrm{e}}$ & $980.4^{\mathrm{k}}$ & -39.6 & $923^{k}$ & -97 & $1141^{\mathrm{k}}$ & 121 \\
\hline & Poisson's ratio & $0.165^{\mathrm{e}}$ & 0.183 & 0.018 & 0.151 & -0.014 & 0.126 & -0.039 \\
\hline & RMS error & - & - & 40.1 & - & 50.1 & - & 80.3 \\
\hline & MAP error & - & - & 26.2 & - & 23.8 & - & 46.9 \\
\hline \multirow[t]{10}{*}{ Graphite } & $E_{\text {Corr }}(\mathrm{eV} /$ atom $)$ & $7.371^{\mathrm{a}}$ & 7.3853 & 0.0143 & 7.372 & 0.001 & 7.311 & -0.060 \\
\hline & Lattice const., a $(\AA)$ & $2.462^{\mathrm{c}}$ & 2.469 & 0.007 & 2.47 & 0.008 & 2.418 & -0.042 \\
\hline & Lattice const., c ( $\mathrm{A})$ & $6.707^{c}$ & 6.694 & -0.013 & 6.71 & 0.003 & 6.713 & 0.008 \\
\hline & $C_{11}+C_{12}(\mathrm{GPa})$ & $1240^{\mathrm{f}}$ & 1184 & -56 & 1052 & -188 & 1309 & 69 \\
\hline & $C_{66}(\mathrm{GPa})$ & $440^{\mathrm{f}}$ & 461 & 21 & 406 & -34 & 508 & 69 \\
\hline & $C_{13}(\mathrm{GPa})$ & $15^{\mathrm{f}}$ & 20 & 5 & 28 & 13 & 0.3 & -14.7 \\
\hline & $C_{33}(\mathrm{GPa})$ & $36.5^{\mathrm{f}}$ & 36.6 & 0.1 & 37.1 & 0.6 & 41.0 & 4.5 \\
\hline & $C_{44}(\mathrm{GPa})$ & $4.5^{\mathrm{f}}$ & 8.6 & 4.1 & 293.6 & 289.1 & 0.1 & -4.4 \\
\hline & RMS error & - & - & 21.3 & - & 122.6 & - & 34.7 \\
\hline & MAP ${ }^{\mathrm{l}}$ error & - & - & 21.7 & - & 817.0 & - & 29.0 \\
\hline
\end{tabular}




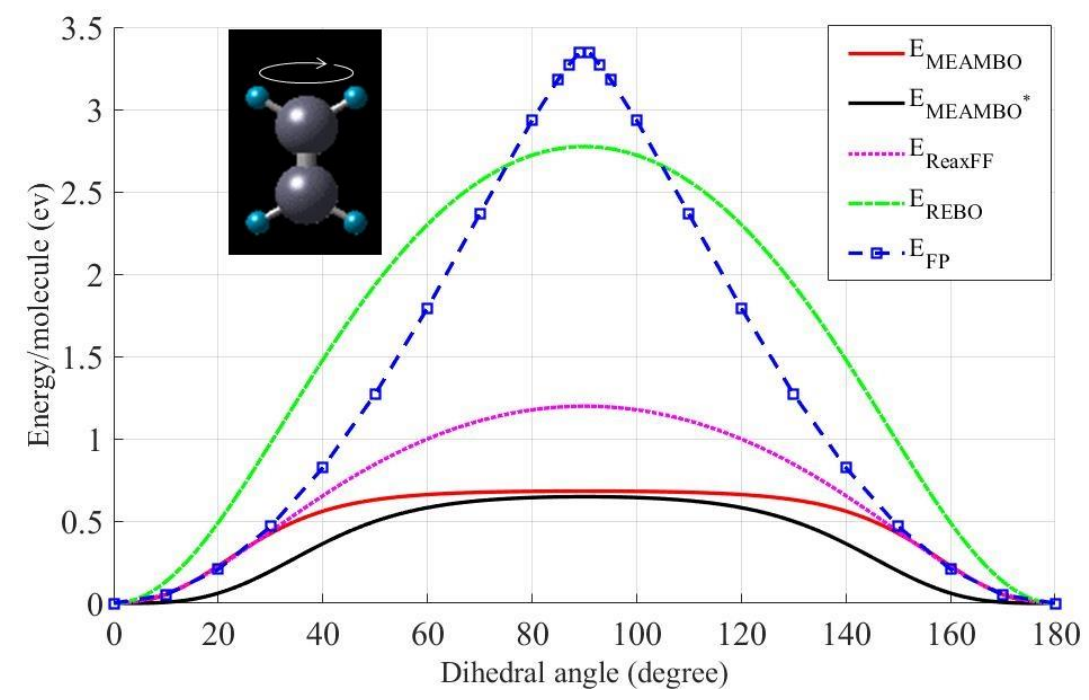

Figure A.1 The rotational barrier for ethylene calculated using MEAMBO- (original/current) and FP using CCSD(2)/aug-cc-pVTZ. ${ }^{145,146}$ The white arrows in the pictures of the molecules indicate the coordinate that is being varied, and the line that passes through the FP or experimental data serves as a guide. The energy is relative to the minimum energy. 